\title{
Recycler Ring Conceptual Design Study
}

\author{
G. Jackson, Editor \\ Fermi National Accelerator Laboratory \\ P.O. Box 500, Batavia, Illinois 60510
}

July 18, 1995 


\section{Disclaimer}

This report was prepared as an account of work sponsored by an agency of the United States Government. Neither the United States Government nor any agency thereof, nor any of their employees, makes any warranty, express or implied, or assumes any legal liability or responsibility for the accuracy, completeness, or usefulness of any information, apparatus, product, or process disclosed, or represents that its use would not infringe privately owned rights. Reference herein to any specific commercial product, process, or service by trade name, trademark, manufacturer, or otherwise, does not necessarily constitute or imply its endorsement, recommendation, or favoring by the United States Government or any agency thereof. The views and opinions of authors expressed herein do not necessarily state or reflect those of the United States Government or any agency thereof. 
Recycler Ring

Conceptual

Design Study

Fermi National Accelerator Laboratory

May 15, 1995 


\section{Recycler Ring Conceptual Design Study Outline}

1. Introduction

1. Overview

2. Luminosity and Stack Size

3. Luminosity Lifetime and Stacking Rate

4. Parameter Lists

5. Symbol Definitions

2. Stacking Antiprotons

1. Antiproton Production and Lithium Lens System

2. Debuncher

3. Accumulator

4. Recycler

5. Symbol Definitions

3. Recycling Antiprotons

1. Evolution of the Luminosity

2. Optimum Store Length

3. Tevatron Requirements

4. Main Injector Requirements

5. Booster Requirements

6. Antiproton Source Requirements

7. Recycler Requirements

8. Symbol Definitions

4. Recycler Design

1. Permanent Magnet Design

1. Major Design Choices

2. Field Strength

3. Aperture and Field Quality Requirements

4. Choice of Permanent Magnet Material

5. Combined Function Lattice Magnets

6. Bar Stock Construction

7. Magnet Support

8. Magnet and Lattice

9. Temperature Compensation of Strontium Ferrite

10. Unique Problems of Permanent Magnets

11. Prototype Program

2. Tunnel Placement of Ring

3. Lattice

4. Electron Cooling

1. Introduction to Electron Cooling

2. Cooling Time for Electron Cooling

4. Electron Cooling and Stacking in the Recycler

5. Hardware

6. Development Program

7. Civil Construction for Electron Cooling 
5. Vacuum Requirements

1. Vacuum Criterion

2. Vacuum Theory

3. Lumped Ion Pump System

4. Vacuum Test

5. In-situ Baking

6. Anticipated Ion Pump Performance

6. Injection and Extraction

7. RF System

8. Dampers and Feedback

9. Beam Instrumentation

10. Correction Systems

11. Intrabeam Scattering

12. Instabilities 


\section{Introduction}

The Tevatron Collider provides the highest center of mass energy collisions in the world. To fully exploit this unique tool, Fermilab is committed to a program of accelerator upgrades for the purpose of increasing the Collider luminosity. Over the past 7 years the luminosity has been increased from a peak of $1.6 \times 10^{30} \mathrm{~cm}^{-2} \mathrm{sec}^{-1}$ in 1989 to over $3 \times 10^{31} \mathrm{~cm}^{-2} \mathrm{sec}^{-1}$ during 1995 . The Main Injector will supply a larger flux of protons for antiproton production and more intense proton bunches for use in the Collider; and this is expected to increase the peak luminosity to close to $1 \times 10^{32} \mathrm{~cm}^{-2} \mathrm{sec}^{-1}$. Further increases in luminosity will require additional upgrades to the Fermilab accelerator complex. This report documents the design of a new fixed-energy storage ring to be placed in the Main Injector tunnel which will provide an initial factor of 2 increase to $2 \times 10^{32} \mathrm{~cm}^{-2} \mathrm{sec}^{-1}$, and ultimately provide the basis for an additional order of magnitude luminosity increase up to $1 \times 10^{33} \mathrm{~cm}^{-2} \mathrm{sec}^{-1}$.

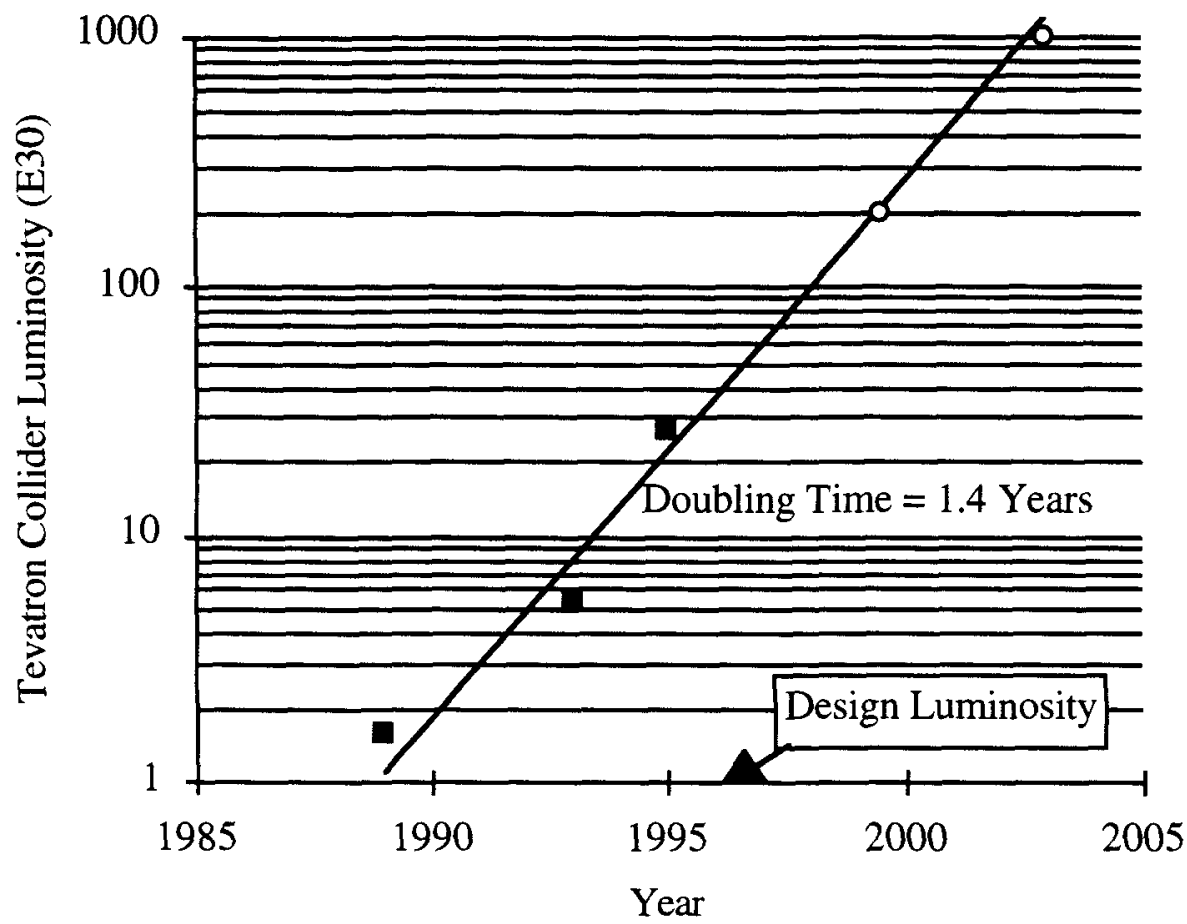

Figure 1.1.1: Tevatron Collider luminosity as a function of time. The filled circles are measured "best typical" peak luminosities, the line is an exponential fit to the data, and the open points represent goals for the future.

Figure 1.1.1 displays the history of the peak luminosity as a function of time since 1989, which shows an exponential growth with a doubling time of less than 18 months. With the addition of the Recycler ring and its commissioning along with the Main Injector, the Tevatron Collider will be able to remain on this exponential slope. The lower open point represents the initial goal of a luminosity of $2 \times 10^{32} \mathrm{~cm}^{-2} \mathrm{sec}^{-1}$ during Collider Run 2. This project, which has been named "Tevatron*" is the subject of this report. It 
should be possible, with further accelerator upgrades, to achieve a luminosity of $1 \times 10^{33} \mathrm{~cm}^{-2} \mathrm{sec}^{-1}$, the upper open point in figure 1.1.1.

\subsection{Overview}

In the first phase, which is the subject of this report, the goal is to reach a peak luminosity of at least $2 \times 10^{32} \mathrm{~cm}^{-2} \mathrm{sec}^{-1}$ within the first year of operation of the Main Injector (Collider Run II). Additional accelerator improvements, which are outside the scope of this report, will yield a luminosity of $1 \times 10^{33} \mathrm{~cm}^{-2} \mathrm{sec}^{-1}$ a few years later. The new storage ring, called the Recycler, will operate at a fixed kinetic energy of $8 \mathrm{GeV}$ and will be built using permanent magnets. It will accept antiprotons from the existing Antiproton Source and will contain an electron cooling system. It will also accept antiprotons that remain in the Tevatron at the end of a store and recool them for use in the next store, thereby increasing the peak and average luminosities possible with a given antiproton production rate. A versatile low-level RF system, together with the very small longitudinal emittance of the electron-cooled antiproton beam, will allow flexible preparation of bunches for injection into the Tevatron via the Main Injector, eliminating the need for coalescing in the Main Injector and allowing almost any combination of buckets in the Tevatron to be populated.

The luminosity of the Tevatron Collider is ultimately determined by the number of antiprotons available for collisions. In the current mode of operation, antiprotons are produced by targeting a $120 \mathrm{GeV}$ proton beam from the Main Ring (and later the Main Injector) and collecting antiprotons in the two rings of the Antiproton Source. Within the Antiproton Source rings (Debuncher and Accumulator) the phase space density of the antiprotons is increased using stochastic cooling systems. Periodically a fraction (typically about $50 \%$ ) of the antiprotons are extracted from the Accumulator, transferred into the Tevatron, and brought into collision with a proton beam at a total center of mass energy of $1.8 \mathrm{TeV}$. During the store additional antiprotons are accumulated in the Accumulator. At the end of the store, typically 10-20 hours in duration, the remaining antiprotons, of order $60 \%$ or more of the original number, are dumped and a new batch is extracted from the Antiproton Source.

Currently the rate of accumulation of antiprotons is limited, at small antiproton stack size, to about $7 \times 10^{10}$ /hour by apertures, cooling systems, and the number of protons on target. The Main Injector will provide a more than 50\% increase in the number of protons per pulse with a $30 \%$ faster repetition rate, yielding a stacking rate in excess of $15 \times 10^{10}$ /hour. Planned improvements to the Debuncher and Accumulator stochastic cooling systems will support a stacking rate of at least $20 \times 10^{10} /$ hour, which could be possible if the Main Injector exceeds its design intensity or if proposed modifications that will increase the momentum acceptance of the Debuncher are implemented. However, because the cooling rate decreases as the stack size increases, going roughly as $1 / \mathrm{N}_{\mathrm{a}}$ (where $\mathrm{N}_{\mathrm{a}}$ is the number of antiprotons), the stacking rate decreases for larger stacks. A high stacking rate can be maintained by performing frequent transfers of antiprotons to the new, larger ring. In this case the Accumulator serves principally to cool the antiproton beam sufficiently that it can be cooled efficiently by the electron cooling system in the Recycler. Because the electron cooling rate is independent of antiproton stack size, substantially larger stacks can be accumulated in the Recycler than in the Accumulator. 
Under current conditions, at least $60 \%$ of the antiprotons initially injected into the Tevatron survive to the end of even a long ( $>20$ hour) store. However, the phase space density of the remaining beam is low due to emittance growth during the store. At the higher luminosities that will be typical in the future, the emittance growth time will be shorter due to intrabeam scattering. This means that if a store is terminated when the luminosity has dropped to $40-50 \%$ of its original value (typical of current operation) an even larger fraction of the original antiprotons will remain than is now the case. For example, under conditions that yield an initial luminosity of $2 \times 10^{32} \mathrm{~cm}^{-2} \mathrm{sec}^{-1}, 4$ hours into the store the luminosity has dropped to half its original value but $80 \%$ of the antiprotons remain in the Tevatron. With presently operational systems it is not practical to reuse these antiprotons because of the relatively long recooling time for large antiproton stacks using stochastic cooling. However, the electron cooling system to be installed in the Recycler has cooling times measured in minutes or seconds, not hours, so recycling the spent antiproton beam becomes practical. Recycling of antiprotons results in an effective increase in stacking rate of more than a factor of two, depending on the recovery efficiency and lengths of stores.

Because of the small longitudinal emittance of the electron cooled beam, the beam in the Recycler can be manipulated easily with a low level, broad band RF system. This makes it possible to prepare bunches in the Recycler for injection into almost arbitrary configurations of RF buckets in the Tevatron without the need for coalescing in the Main Injector, and to inject the entire antiproton stack into the Tevatron for colliding beams, rather then the roughly $50 \%$ that can be extracted from the Accumulator now. Once the Recycler as been emptied of antiprotons, it can be used to prepare the proton beam in a similar fashion. This ability to prepare both beams provides substantial operational flexibility with respect to choosing the number and spacing of bunches in the Tevatron, in turn allowing these to be optimized to the requirements of the experimental physics program and the capabilities of the detectors.

Ultimately the parameter of most interest is the integrated luminosity logged to tape by the experiments observing the antiproton-proton collisions. Three aspects of the Recycler ring system enhance this parameter. First, by using permanent magnets for the guide and focusing fields, the Recycler will be immune to a number of failures, such as power dips or power supply regulation errors, which can cause the loss of the stack in a ring made of electromagnets. The improved reliability will result in a larger integrated luminosity over a long period. Second, the ability to recycle antiprotons allows the use of shorter stores to obtain the same average luminosity. This reduces the ratio of peak luminosity to average luminosity, which in turn results in more efficient operation of the experiments, since data acquisition dead time grows with increasing luminosity. Third, the ability to transfer $100 \%$ of the available antiproton into either the Tevatron or Recycler allow servicing of either machine without purposely dumping the precious beam.

The following sections of this chapter will give a more quantitative discussion of the operational issues mentioned above, and a parameter list for Collider operations with the Recycler will be presented and compared to present and planned operation without the Recycler. The second chapter of this report will discuss the stacking of antiprotons and the third chapter will discuss the recycling of antiprotons. The Recycler ring and its hardware systems, including lattice, magnets, RF system, vacuum system, electron cooling system, etc., will be the subject of chapter four. 


\subsection{Luminosity and Stack Size}

The luminosity $\mathrm{L}$ per interaction region of a round beam proton-antiproton collider is

$$
L=\frac{N_{p}\left(N_{a} B\right) f_{o}\left(\beta_{r} \gamma_{r}\right)}{2 \pi \beta^{*}\left(\varepsilon_{n p}+\varepsilon_{n a}\right)} \cdots
$$

where $N_{p, a}$ is the number of protons and antiprotons per bunch, $B$ is the number of bunches per beam, $f_{0}$ is the revolution frequency of the Tevatron, $\beta_{r}$ and $\gamma_{r}$ are the relativistic velocity and energy of the beam, $\beta^{*}$ is the value of the beta function at the interaction points, and $\varepsilon_{\mathrm{np}, \mathrm{na}}$ are the proton and antiproton rms normalized transverse emittances. Additional factors which reduce the luminosity due to crossing angles between the beams or bunch lengths longer than $\beta^{*}$ are indicated by the ellipses and are not shown explicitly, and have a typical value of $2 / 3$. The quantity $N_{a} B$ (called the stack size) is just the total antiproton intensity injected into the Tevatron Collider, independent of the number of bunches that charge is divided into. The limit on the proton intensity comes from the observation that the maximum allowable total antiproton linear beam-beam tune shift from all bunch crossings is approximately 0.026 . The equation relating this maximum total tune shift $\xi_{\max }$ and the proton intensity is

$$
\xi_{\max }=\frac{\mathrm{r}_{0}}{4 \pi} \frac{\mathrm{N}_{\mathrm{p}}}{\varepsilon_{\mathrm{np}}} \mathrm{N}_{\mathrm{IR}}
$$

The quantity $r_{0}$ is the classical radius of the proton $\left(1.53 \times 10^{-18} \mathrm{~m}\right)$. The number of interaction regions $\mathrm{N}_{I R}$ is at a minimum equal to the number of high energy physics detectors operating in the collider. Plugging this equation for the tune shift into the equation for luminosity per interaction region (1.2.1) yields the result

$$
L=\frac{\left(N_{\mathrm{a}} \mathrm{B}\right)}{\mathrm{N}_{\mathrm{IR}} \beta^{*}} \frac{2 \xi_{\max } \mathrm{f}_{\mathrm{o}}\left(\beta_{\mathrm{r}} \gamma_{\mathrm{r}}\right)}{\mathrm{r}_{\mathrm{o}}\left(1+\frac{\varepsilon_{\mathrm{na}}}{\varepsilon_{\mathrm{np}}}\right)}
$$

where the factors whose values can be significantly modified appear in the left fraction. The emittances of the protons and antiprotons can be changed, but the ratio of the proton to antiproton emittances is always near unity due to nonlinear beam dynamics concerns. Therefore, reducing the emittances of the beams is not helpful once the beam-beam limit has been attained.

Even though $\beta^{*}$ can be changed, eventually the length of the bunch and the sensitivity of the lattice to errors limits the extent to which $\beta^{*}$ can be reduced. The number of interaction regions is chosen by the high energy physics community (by choosing the number of bunches). Note that the sum of the luminosity available in the Tevatron Collider at all interaction regions is conserved as the number of interaction regions is changed. 
Table 1.2.1: Values of variable found in equation (1.2.3) used to generate the plot in figure 1.2.1.

\begin{tabular}{|l|c|}
\hline Parameters & Value \\
\hline \hline$\left(\beta_{\mathrm{r}} \gamma_{\mathrm{r}} \quad\right.$ (Beam Energy =1 TeV) & 1066 \\
\hline$\beta^{* \quad \text { Interaction Point Beta }(\mathrm{cm})}$ & 35 \\
\hline$\varepsilon_{\mathrm{np}} / \varepsilon_{\mathrm{na}}$ Ratio of Beam Emittances & 1 \\
\hline $\mathrm{N}_{\mathrm{IR} \quad \text { Number of Interaction Regions }}$ & 2 \\
\hline$\xi_{\mathrm{max}} \quad$ Maximum Antiproton Tune Shift & 0.024 \\
\hline $\mathrm{f}_{\mathrm{O}} \quad$ Revolution Frequency $(\mathrm{kHz})$ & 47.7 \\
\hline
\end{tabular}

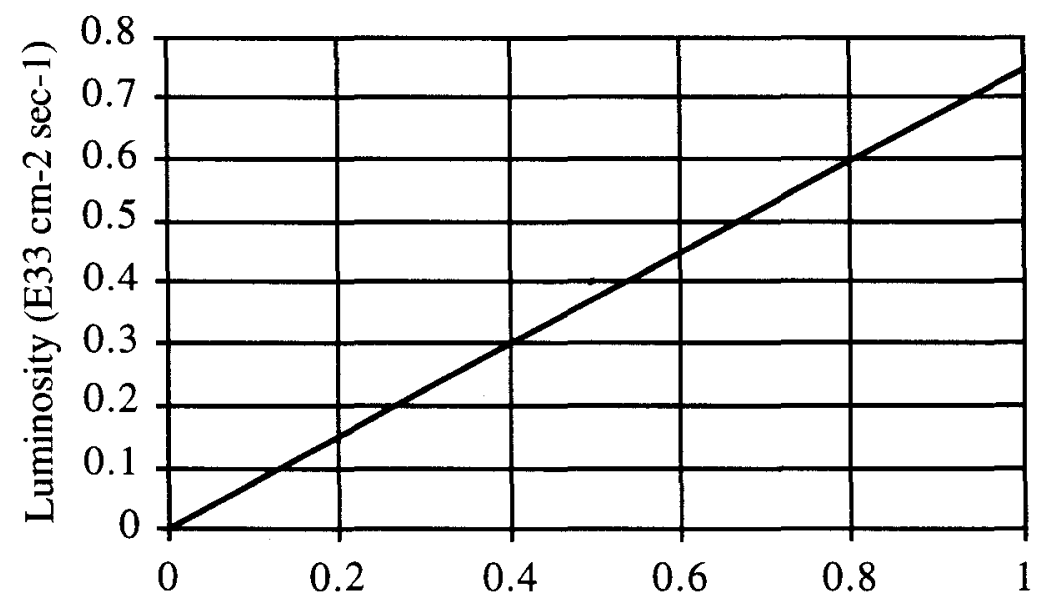

Antiproton Stack Size (E13)

Figure 1.2.1: Calculation of the Tevatron Collider luminosity as a function of antiproton stack size given the parameter values listed in table 1.2.1.

Using the values in table 1.2.1 for the variables appearing in equation (1.2.3), figure 1.2.1 shows the luminosity per interaction region as a function of the antiproton stack size. Note that for the Tevatron* luminosity goal of $2 \times 10^{32} \mathrm{~cm}^{-2} \mathrm{sec}^{-1}$, the required amount of antiprotons at the beginning of the store is $2 \times 10^{12}$, or $200 \mathrm{~mA}$ of Accumulator current. Using the old method of Accumulator beam extraction in which approximately half of the antiproton stack is extracted for a particular store, a peak Accumulator current of $400 \mathrm{~mA}$ would be required. While this size stack might be possible in the Accumulator given a number of technically risky upgrades (such as $8-16 \mathrm{GHz}$ cooling systems), the antiproton intensity of $1 \times 10^{13}$ required by Tevatron33 is not feasible. Use of the Recycler ring will greatly increase the maximum stack that can be stored and eliminate this limitation.

\subsection{Luminosity Lifetime and Stacking Rate}

The central purpose of the Tevatron Collider is to collide protons and antiprotons at high luminosity in order to accumulate as much integrated luminosity as possible in as little time as possible. One of the repercussions of high luminosity is that particles are consumed at the rate of 


$$
\mathrm{R}_{\text {lost }}=-\mathrm{N}_{\mathrm{IR}} \mathrm{L} \sigma_{\text {lost }}
$$

where $\sigma_{\text {lost }}$ is the cross-section for losing protons and antiprotons due to both inelastic and large elastic interactions and $\mathrm{N}_{\mathrm{IR}}$ is the number of interaction regions in the Collider. The proton and antiproton intensity lifetimes due to this mechanism are calculated using the equation

$$
\tau_{\mathrm{Lp}, \mathrm{La}}=-\frac{N_{\mathrm{p}, \mathrm{a}} \mathrm{B}}{\mathrm{R}_{\text {lost }}}
$$

The luminosity lifetime $\tau_{\mathrm{L}}$ is defined as

$$
\frac{1}{\tau_{\mathrm{L}}}=\frac{1}{\mathrm{~L}} \frac{\mathrm{dL}}{\mathrm{dt}}
$$

Substituting equation (1.3.1) into (1.2.3) and performing the derivative on all quantities known to change during a Tevatron Collider store yields the result

$$
\frac{1}{\tau_{L}}=\frac{1}{N_{p}} \frac{d N_{p}}{d t}+\frac{1}{N_{a}} \frac{d N_{a}}{d t}-\frac{1}{\varepsilon_{n p}} \frac{d \varepsilon_{n p}}{d t}
$$

where it has been assumed (consistent with standard Tevatron Collider operations) that the proton and antiproton emittances are equal and proton and antiproton emittance growth rates are equal. For luminosity of $1 \times 10^{32} \mathrm{~cm}^{-2} \mathrm{sec}^{-1}$ or higher and beam parameters anticipated for the Tevatron Collider using the Recycler ring with electron cooling to prepare the beam, the initial luminosity lifetime is dominated by emittance growth due to intrabeam scattering, while after several hours the effect of antiproton loss due to luminosity becomes relatively more important. Using the values in table 1.4.1, the evolution of luminosity (figure 1.3.1), proton and antiproton intensity (figure 1.3.2), and beam emittance and bunch area (figures 1.3.3 and 1.3.4) are calculated as a function of time for the case of initial luminosity of $2 \times 10^{32} \mathrm{~cm}^{-2} \mathrm{sec}^{-1}$. The calculation includes the effect beam loss due to luminosity, intrabeam scattering, and noise induced transverse and longitudinal emittance growth. When compared with the evolution of high luminosity stores $\left(\sim 2 \times 10^{31} \mathrm{~cm}^{-2} \mathrm{sec}^{-1}\right)$ during Collider Run $\mathrm{Ib}(1995)$ the calculation agrees well. 


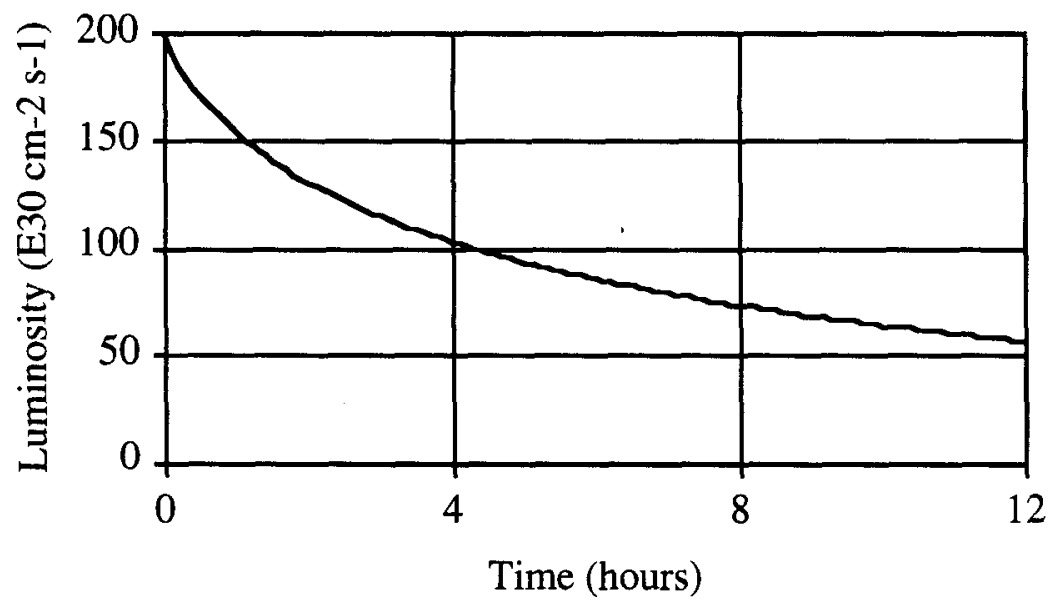

Figure 1.3.1: Luminosity as a function of time in the Tevatron* scenario. The parameters used to perform this calculation are listed in the Tev* column of table 1.4.1.

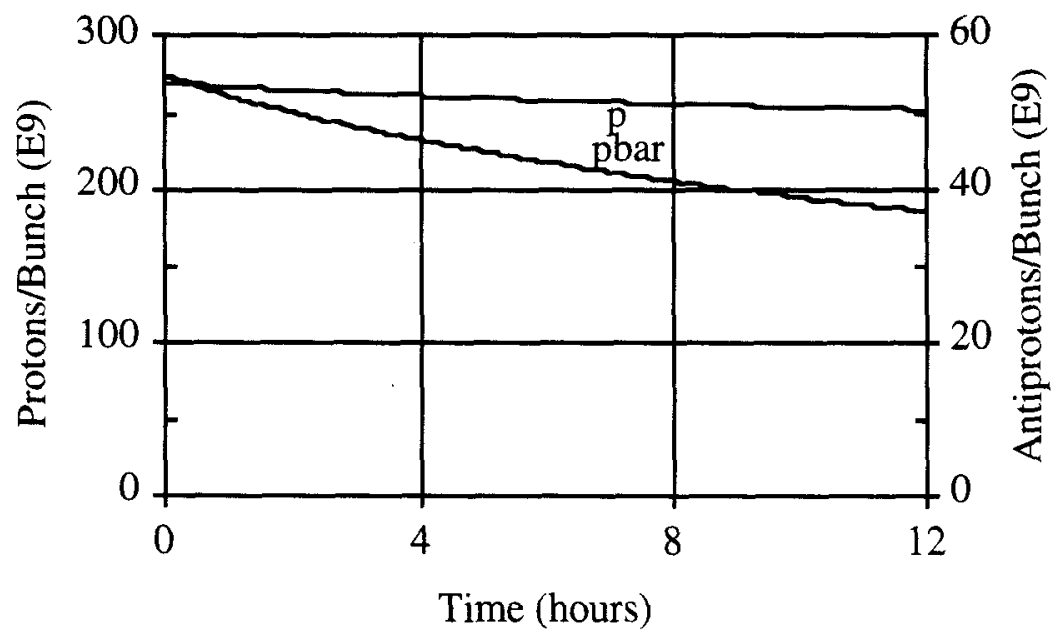

Figure 1.3.2: Proton and antiproton intensity per bunch as a function of time in the Tevatron* scenario. The parameters used to perform this calculation are listed in the Tev* column of table 1.4.1. 


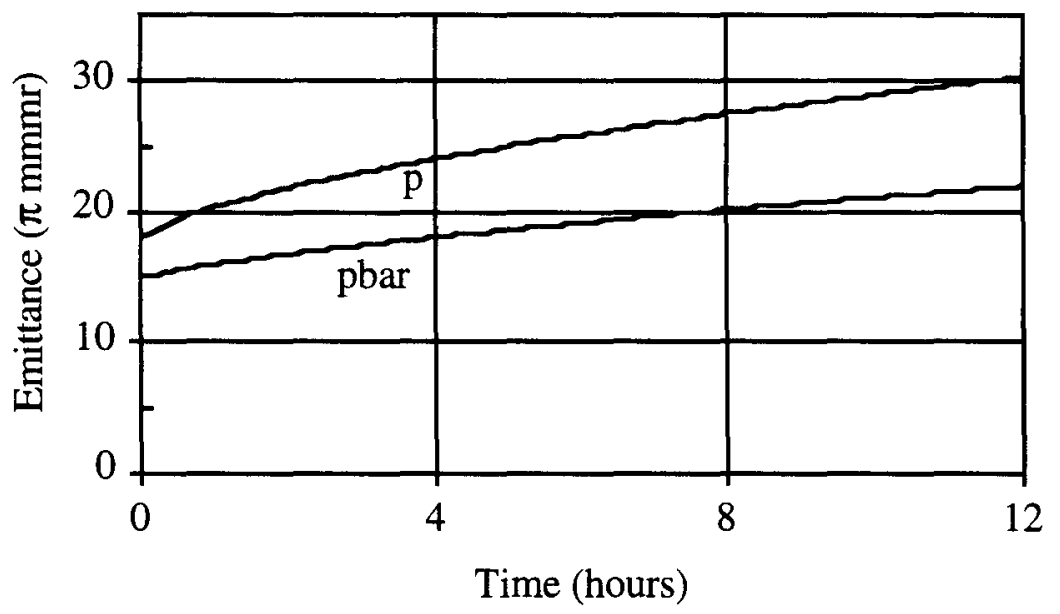

Figure 1.3.3: Transverse emittances as a function of time in the Tevatron* scenario. The parameters used to perform this calculation are listed in the Tev* column of table 1.4.1.

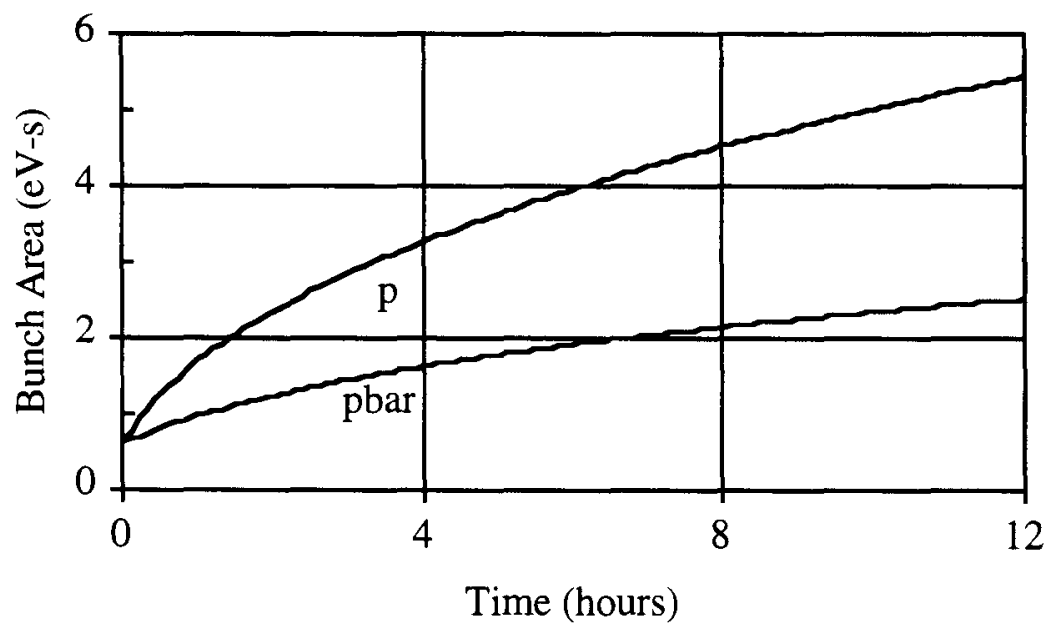

Figure 1.3.4: Longitudinal emittances as a function of time in the Tevatron* scenario. The parameters used to perform this calculation are listed in the Tev* column of table 1.4.1. 


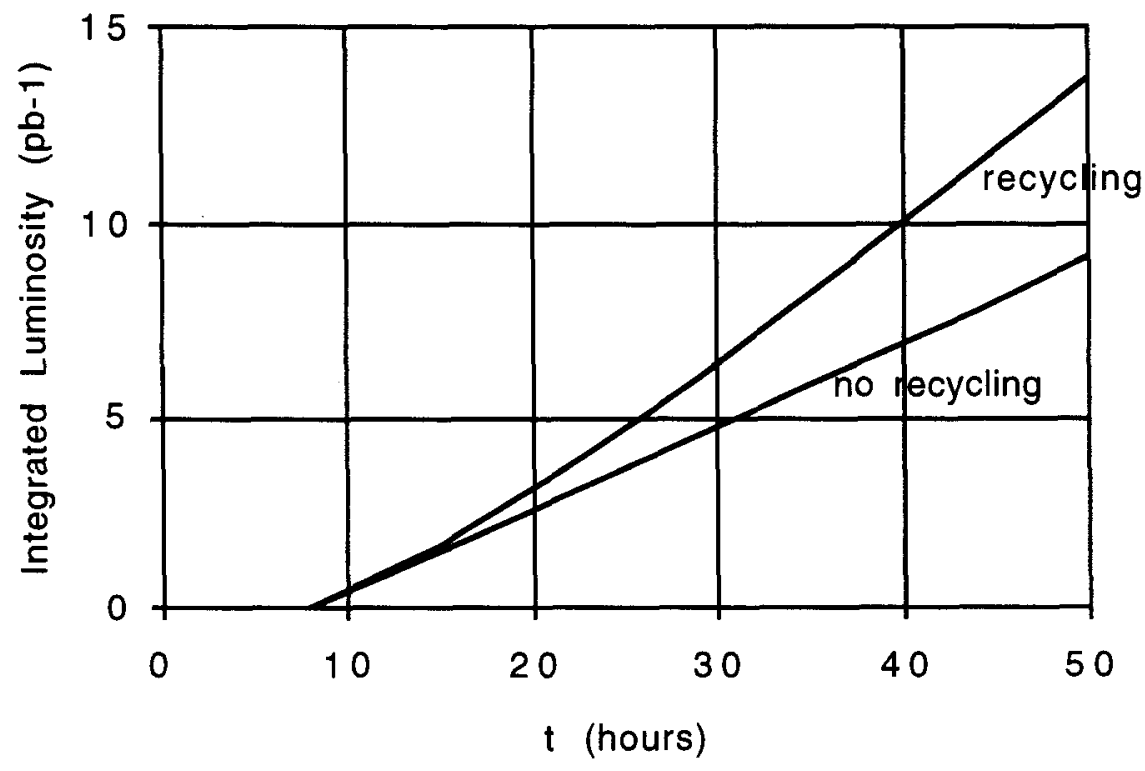

Figure 1.3.5: Integrated luminosity for two days of steady "stack and store" operation, with and without antiproton recycling. A stacking rate of $15 \times 10^{10}$ /hour and a 1 hour shot setup time have been assumed.

The initial luminosity lifetime is quite short, about 2.5 hours, but grows rapidly to almost than 10 hours after 4 hours. The luminosity drops to half its initial value after 3.5 hours and to $1 / \mathrm{e}$ of it original value after about 6.5 hours. This suggests that store durations of 4-6 hours will be optimal. At the end of a 6 hour store $80 \%$ of the original antiproton beam remains in the Tevatron, but with transverse emittance increased by $25 \%$ and bunch area more than 3 times its initial value. Without the Recycler ring, the remaining antiprotons must be dumped, but with the Recycler they can be quickly recooled for use in the next store. Figure 1.3.5 compares the evolution of integrated luminosity over a two day period, assuming a constant stacking rate of $15 \times 10^{10}$ hour, a $90 \%$ recycling efficiency, and an 8 hour initial stacking period, with and without antiproton recycling. A 6 hour store duration has been assumed for the recycling case and 8 hours without, and a 1 hour shot setup time is assumed. Note that as stores become shorter, it will become increasingly important to shorten the inter-store period. After two days the integrated luminosity is more than $60 \%$ higher due to recycling.

\subsection{Parameter Lists}

Table 1.4.1 contains a summary of the operational parameters both observed in the past and expected in the future. All beam and instantaneous luminosity parameters are evaluated at the beginning of a typical store. The integrated luminosity per week assumes the standard Snowmass criterion that the collider operates at the peak luminosity for a third of the hours in that week. The origin of the expected performance numbers for future stores will be explained later in this report. The column named MI (Main Injector) lists the design 
parameters of the original Main Injector project before the existence of the Recycler ring was considered.

Table 1.4.1: Values of Tevatron Collider parameters in past and future runs. The emittances are $95 \%$ normalized values normally used by Fermilab accelerator staff. All instantaneous parameters are evaluated at the beginning of a typical store.

\begin{tabular}{|c|c|c|c|c|c|c|}
\hline Parameter & $1988 / 9$ & $1992 / 3$ & 1995 & $\overline{\mathrm{MI}}$ & Tev* & Tev33 \\
\hline Protons/Bunch $\left(10^{9}\right)$ & 70 & 120 & 225 & 330 & 270 & 270 \\
\hline Antiprotons/Bunch $\left(10^{9}\right)$ & 29 & 31 & 65 & 36 & 55 & 66 \\
\hline Proton Emittance $(\pi \mathrm{mmmr})$ & 25 & 20 & 22 & 30 & 18 & 18 \\
\hline Antiproton Emittance $(\pi \mathrm{mmmr})$ & 18 & 12 & 14 & 20 & 15 & 15 \\
\hline Beta@ IP (cm) & 55 & 35 & 35 & $\overline{35}$ & 35 & 25 \\
\hline Beam Energy $(\mathrm{GeV})$ & 900 & 900 & 900 & 1000 & 1000 & 1000 \\
\hline Bunches/Beam & 6 & 6 & 6 & 36 & 36 & 108 \\
\hline Longitudinal Emittance (eV-s) & 6.3 & 4.5 & 3.7 & 3.0 & 0.6 & 0.3 \\
\hline RMS Bunch Length $(\mathrm{cm})$ & 65 & 55 & 50 & 45 & 20 & 15 \\
\hline Interaction Regions & 12 & 2 & 2 & 2 & 2 & 2 \\
\hline Minimum Bunch Spacing (ns) & 3500 & 3500 & 3500 & 395 & $395(132)$ & 132 \\
\hline Luminosity Form Factors & $\overline{0.71}$ & $\overline{0.62}$ & 0.62 & 0.70 & $\overline{0.88}$ & 0.88 \\
\hline Luminosity $\left(10^{30} \mathrm{~cm}^{-2} \mathrm{~s}^{-1}\right)$ & 1.60 & 5.42 & 19 & 83 & 200 & 1000 \\
\hline Integrated Lum (pb-1/week) & 0.32 & 1.1 & 4 & 17 & 40 & 200 \\
\hline Interactions/Crossing (@ 45mb) & 0.25 & $0 . \overline{85}$ & 3.0 & 2.2 & 5.2 & 8.7 \\
\hline Total Antiproton Tune Shift & 0.026 & 0.009 & 0.015 & 0.016 & 0.023 & 0.023 \\
\hline Total Proton Tune Shift & 0.015 & 0.004 & 0.007 & 0.003 & 0.006 & 0.007 \\
\hline Antiproton Intensity $\left(10^{10}\right)$ & 17 & 19 & 39 & 130 & 198 & 710 \\
\hline Loss Rate $\left(10^{10} / \mathrm{hr} @ 78 \mathrm{mb}\right)$ & 0.09 & 0.30 & 1.1 & 4.7 & 11 & 56 \\
\hline Scenario & actual & actual & actual & design & goal & goal \\
\hline
\end{tabular}

\begin{tabular}{|l|c|c|c|c|c|c|}
\hline Parameter & $1988 / 9$ & $1992 / 3$ & 1995 & MI & Tev $^{*}$ & Tev33 \\
\hline \hline Store Duration (hrs) & & & 12 & 12 & 6 & 4 \\
\hline Antiproton Remaining (\% Inj.) & & & 73 & 65 & 79 & 81 \\
\hline Recycling Efficiency (\%) & & & 0 & 0 & 80 & 80 \\
\hline Injection Eff. to LowBeta (\%) & & & 75 & 90 & 90 & 90 \\
\hline Usable Stack Required (E10) & & & 64 & 144 & 220 & 792 \\
\hline Antiprotons Recycled (E10) & & & 0 & 0 & 125 & 464 \\
\hline New Antiprotons Required (E10) & & & 64 & 144 & 95 & 328 \\
\hline Required Stacking Rate (E10/hr) & & & 5.3 & 12 & 16 & 82 \\
\hline Average Luminosity (pb-1/hour) & & & 0.05 & 0.21 & 0.39 & 1.74 \\
\hline
\end{tabular}

The relationship between the columns listed 1995, MI, Tev(atron)*, and Tev(atron)33 are of most interest at this time. The modifications to the accelerator complex underway at present allow the operational transition $1995 \rightarrow \mathrm{MI}$. The purpose of this document is not to describe the path $\mathrm{MI} \rightarrow \mathrm{Tev}^{*}$, but rather the modifications in addition to the present Main Injector project to achieve the transformation $1995 \rightarrow \mathrm{Tev}^{*}$. The upgrade Tev* $\rightarrow$ Tev33 will be accomplished by putting more bunches into the Tevatron and stacking more antiprotons by further upgrades to the Antiproton Source. These upgrades are beyond the scope of this 
report, but the higher luminosity parameters are included to indicate the sort of further luminosity improvements that are made possible by use of the Recycler ring.

Since luminosity depends only on the number of antiprotons available for injection into the Tevatron Collider, the row in table 1.4.1 labeled "Antiproton Intensity" is the first to be reviewed in this discussion. Note that the ratio of Tev* to MI scenarios is only 1.5, seemingly violating the relationship between luminosity and antiproton intensity. The main reason for the breakdown in this scaling rule is the $30 \%$ increase in the luminosity form factor (see the row labeled "Luminosity Form Factors") due to the smaller anticipated bunch lengths in Tevatron* operations (see row labeled "RMS Bunch Length").

Perhaps the biggest change between the Main Injector and Tevatron* operational scenarios is in the subject of collider bunch preparation. In the case of the Main Injector design the process of coalescing 13 separate $53 \mathrm{MHz}$ bunches into a single collider bunch is retained from current operating procedures. When the Recycler ring is used to electron cool both antiprotons and protons, the collider bunches will be formed at $8 \mathrm{GeV}$ in the Recycler by using a broadband RF system. Since the longitudinal acceptance of the Main Injector is $0.5 \mathrm{eV}-\mathrm{s}$, collider bunches with that emittance or lower can be transmitted to the Tevatron. The row labeled "Longitudinal Emittance" in table 1.4.1 lists the past, present, and expected future longitudinal bunch emittances. The detailed explanation of how this bunch preparation in the Recycler may be achieved appears later in this report.

Given that the luminosity in equation (1.2.3) is unaffected when the number of bunches per beam is changed, injecting more bunches yields fewer interactions per crossing for the same luminosity, which is desirable from the standpoint of precision event reconstruction. However, the drift chambers, calorimeters, and electronics in the high energy physics detectors cannot operate at arbitrarily short times between the bunch crossings. Presently contemplated upgrades of the two detectors CDF and D0 will allow for 108 bunch operation (see row labeled "Bunches/Beam) with a bunch spacing of $132 \mathrm{~ns}$. As described later in this report, minimum bunch separations of $56.4 \mathrm{~ns}, 37.6 \mathrm{~ns}$, and even $18.8 \mathrm{~ns}$ are possible as luminosity increases toward the Tevatron 33 goal. The bunch spacings actually used will represent a balance between the desire for a small number of interactions per crossing and the cost of implementing detector upgrades to accommodate shorter bunch spacing. At bunch separations of 132 ns and smaller accelerator issues such as crossing angles, small emittance preservation, and beam instabilities will also become important.

\subsection{Symbol Definitions}

B Number of bunches per beam

$\beta^{*} \quad$ Beta function value at the Tevatron Collider interaction points

$\beta_{\mathrm{r}} \quad$ Relativistic beam velocity

$\varepsilon_{\text {na }} \quad$ Rms normalized transverse antiproton emittance

$\varepsilon_{\mathrm{np}} \quad$ Rms normalized transverse proton emittance

$\mathrm{f}_{\mathrm{o}} \quad$ Revolution frequency of an accelerator

$\gamma_{\mathrm{r}} \quad$ Relativistic beam energy

L Instantaneous luminosity during a store 
$\mathrm{n}_{\mathrm{L}} \quad$ Number of interactions in a given bunch crossing at an interaction point

$\mathrm{N}_{\mathrm{a}} \quad$ Number of antiprotons per bunch

$\mathrm{N}_{\mathrm{p}} \quad$ Number of protons per bunch

$\mathrm{N}_{\mathrm{IR}}$ Number of interaction regions in the Tevatron Collider

$\mathrm{R}_{\text {lost }} \quad$ Rate at which particles are lost due to proton-antiproton collisions

$r_{0} \quad$ Classical radius of the proton $\left(=1.53 \times 10^{-18} \mathrm{~m}\right)$

$\sigma_{\text {lost }}$ Cross section for all collision processes which reduce beam intensity

$\tau_{\mathrm{L}} \quad$ Luminosity Lifetime

$\tau_{\mathrm{Lp}} \quad$ Antiproton intensity lifetime due to particle collisions at the interaction points

$\tau_{\mathrm{La}}$ Proton intensity lifetime due to particle collisions at the interaction points

$\xi_{\max }$ Total antiproton linear beam-beam tune shift acceptable for collider operations

$\mathrm{A}_{a} \quad$ Antiproton bunch area (95\% invariant longitudinal emittance)

$A_{p} \quad$ Proton bunch area (95\% invariant longitudinal emittance) 


\section{Stacking Antiprotons}

Tevatron Collider luminosity is proportional to the initial antiproton stack size, which in turn depends on the rate at which antiprotons are stacked. Therefore it is essential to understand how to generate high stacking rates to support the Tevatron* luminosity goal of $2 \times 10^{32} \mathrm{~cm}^{-2} \mathrm{sec}^{-1}$. Although they are not within the scope of the Tevatron* upgrades, the Antiproton Source upgrades described in this section will take place in parallel in the form of Accelerator Improvement Projects (AIP) already planned in conjunction with the Main Injector project.

The antiproton stacking rate achievable with the Main Ring at this time at small Accumulator stack sizes is $7 \times 10^{10} / \mathrm{hr}$. The stacking rate required to reliably attain the present luminosity is calculated to be $5.3 \times 10^{10} / \mathrm{hr}$. The design average stacking rate for the Main Injector in the absence of the Recycler was $15 \times 10^{10} / \mathrm{hr}$. In order to achieve the Tevatron* luminosity goal of initial luminosity of $2 \times 10^{32} \mathrm{~cm}^{-2} \mathrm{sec}^{-1}$ using 6 hour stores (and therefore 6 hour stacking time) a stacking rate of $16 \times 10^{10} / \mathrm{hr}$, only slightly greater than the Main Injector design rate, is required.

In the Antiproton Source upgrades (in preparation for Run II) the increase in the antiproton stacking rate from $7 \times 10^{10} / \mathrm{hr}$ to $16 \times 10^{10} / \mathrm{hr}$ will be accomplished by means of a number of individual upgrades in the antiproton target system and stochastic cooling improvements in both the Debuncher and Accumulator. In addition the Main Injector will be able to deliver more than twice the number of protons per second to the antiproton production target. The Main Injector will have an intensity of $5 \times 10^{12}$ protons/pulse with a cycle time, when operating purely for antiproton production, of 1.5 seconds, compared to $3.5 \times 10^{12}$ protons/pulse every 2.4 seconds with the Main Ring. The improvements in the Antiproton Source systems described below, which are planned to be implemented as part of the Fermilab III program on the same time scale as the Main Injector and Recycler construction, are sufficient to provide a stacking rate at low stack size of at least $20 \times 10^{10 / h r}$.

\subsection{Antiproton Production Target and Lithium Lens System}

Handling the increased proton intensity on target while maintaining an energy density less than the $1100 \mathrm{~J} / \mathrm{gm}$ which would melt the nickel target is the challenge of Main Injector era targeting. If the differential heating is sufficiently severe, it is possible to crack the target. At higher local temperature, distortion of the target material and shape due to melting could become a problem. If the target material melts and begins to outgas, radioactive heavy metal molecules could be released by the target. At present the energy density is approximately $950 \mathrm{~J} / \mathrm{gram}$ of target material. The more intense pulses from the Main Injector will increase the energy density above the threshold for target melting if measures are not taken to distribute the beam energy in a larger target volume.

There are two methods by which the energy density on the target can be reduced. The first is to defocus the proton beam on the target. Unfortunately, the transverse phase space of the antiprotons is proportional to the product of the antiproton beam divergence in the target (determined solely by the physics of antiproton production) and the proton spot size. Therefore, the capture efficiency will be compromised unless the physical apertures in the transfer lines and the Debuncher are increased. 
The second method is to sweep the proton beam across the target in such a way that within the $1.6 \mu \mathrm{s}$ proton batch a substantially larger area on the target is illuminated. If there is a matching steering system for the antiprotons, the sweeping system would produce a beam with the same transverse phase space area as possible with present low intensity proton batches. For the Main Injector target sweeping of a defocused spot size is the anticipated operation method.

\subsection{Debuncher}

The increased number of antiprotons produced by the Main Injector beam and the shorter cycle time both require an increased cooling rate in the Debuncher. This will be accomplished by two improvements. The first is to reduce the temperature of the pickups from $80 \mathrm{~K}$ to $4 \mathrm{~K}$, resulting in a factor of 6 improvement in signal to noise. The second is to dynamically change the momentum compaction factor between the initial debunching phase and the betatron cooling phase of the Debuncher cycle. The original value of the momentum compaction $\eta=0.006$ was set to ensure adequate bucket height for the initial bunch rotation. Shunts have been placed on the quadrupoles in the Debuncher which allow $\eta$ to be increased to 0.009 after the bunch rotation. This larger value of the momentum compaction factor increases the mixing and therefore the cooling rate. This has been demonstrated during Run Ib.

It is possible to increase the number of antiprotons stacked through the system by increasing the momentum aperture of the Debuncher. This is accomplished by reducing the peak dispersion in the ring. Figure 2.2.1 contains a plot of the dispersion and beta functions in $2 / 3$ of the ring. Using the same lattice design technique presently being employed to change the momentum compaction for bunch rotation and stochastic cooling, quadrupole shunt currents have been calculated to accomplish the reduction in the dispersion function. A comparison of possible momentum acceptance with that of the nominal lattice through a study of the rms beam size indicates a $25 \%$ larger momentum acceptance. In this new lattice the momentum compaction has a value of 0.0029 . Figure 2.2.2 is an example of such a lattice modification.

\subsection{Accumulator}

With the existing $1-2 \mathrm{GHz}$ Accumulator stack-tail cooling system the stacking rate is limited to $14 \times 10^{10} / \mathrm{hr}$ after a stacking efficiency of about $75 \%$. Calculations show that if the system is upgraded to the frequency band $2-4 \mathrm{GHz}$, the efficiency increases to $95 \%$ and the stacking rate improves to a value of $17 \times 10^{10} / \mathrm{hr}$.

The width of a given Schottky band is proportional to its frequency, the momentum spread of the beam being cooled, and the momentum compaction of the accelerator lattice. When Schottky bands overlap, some particles are heated. When the overlap is significant the efficiency of the stack-tail system drops. Therefore, a frequency increase must be balanced by a comparable reduction of the momentum compaction. The relationship between momentum compaction $\eta$, the relativistic beam energy $\gamma_{\mathrm{r}}$, and the transition gamma $\gamma_{t}$ is 


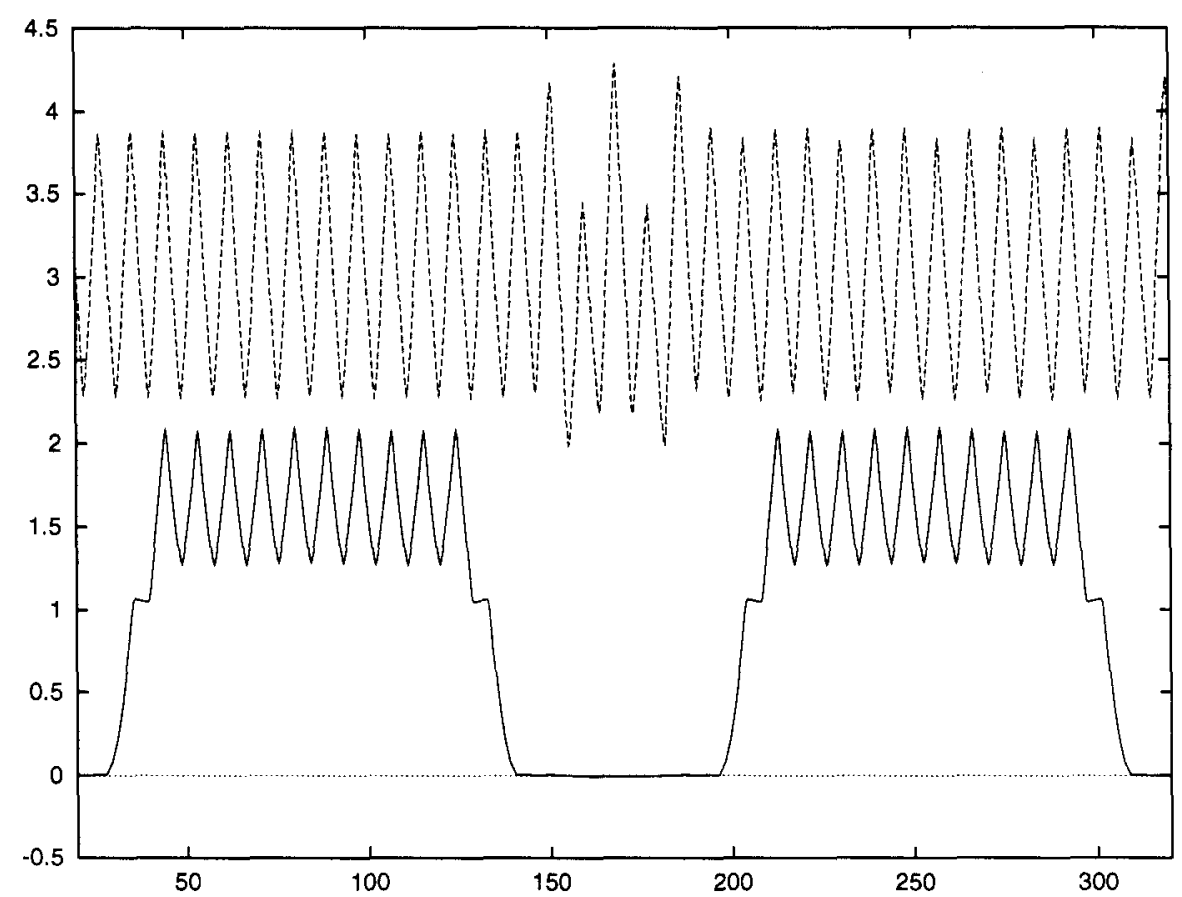

Figure 2.2.1: Nominal horizontal beta function (top dashed line) and dispersion (bottom solid line) in the Debuncher.

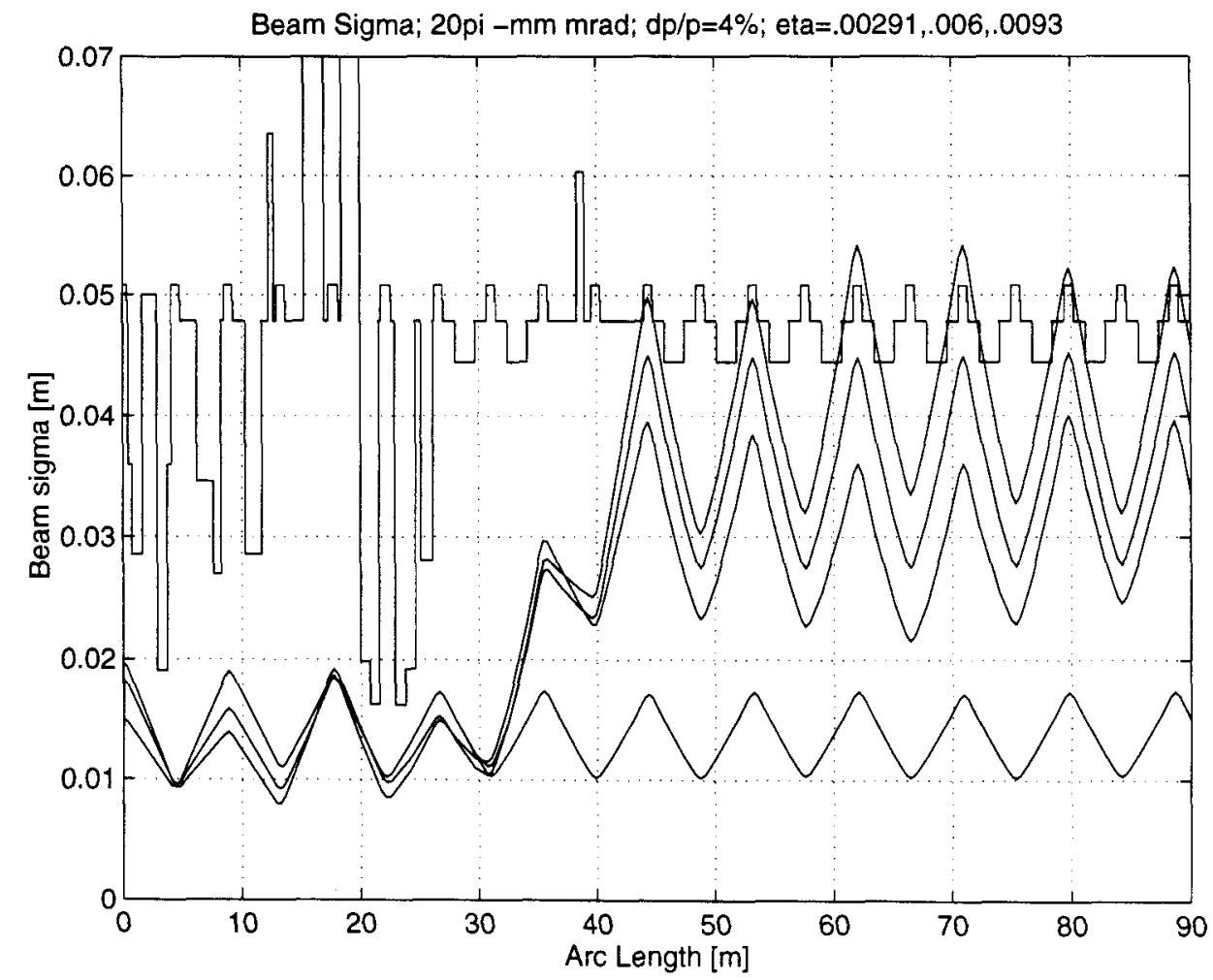

Figure 2.2.2: Example of the rms beam sizes in the Debuncher for three different lattice solutions. The momentum compaction of 0.0029 corresponds to the lowest of the three dispersion curves. 


$$
\eta=\frac{1}{\gamma_{t}^{2}}-\frac{1}{\gamma_{r}^{2}}
$$

To decrease the momentum compaction, it is necessary to raise $\gamma_{\mathrm{t}}$ to a value closer to the relativistic beam energy of 9.529 . The relationship between $\gamma_{t}$ and the Accumulator lattice is

$$
\frac{1}{\gamma_{t}^{2}}=\frac{1}{C} \oint \frac{D(s)}{\rho(s)} d s
$$

The integral around the circumference $C$ of the ratio of the dispersion $D(s)$ to the radius of curvature $\rho(s)$ is simply proportional to the average dispersion in the dipole magnets. Therefore, to increase $\gamma_{t}$ it is necessary to reduce the average dispersion. Unfortunately, due to the restrictions imposed by the already existing lattice, to accomplish this change the peak dispersion increases slightly.

Using the Methodical Accelerator Design (MAD) simulation code, a strategy for raising $\gamma_{t}$ in the Antiproton Source Accumulator ring has been worked out. Each of the six sectors in the Accumulator has fourteen quadrupole magnets. Nine of these are small $\mathrm{TeV}$ I quadrupoles and five are large $\mathrm{TeV}$ I quadrupoles. The largest current increase in any of the small quadrupoles is ten percent. Since there is no evidence of saturation for this change, it is not a problem. Saturation is a problem in the large quadrupoles. The largest current increase in any of the large quadrupoles is seven percent. It will thus be necessary to replace one of the large quadrupoles in each sector with a longer large quadrupole( i.e. one must switch from a LQB to a LQC). The quadrupole current changes are illustrated in table 2.3.1.

In this scheme, $\gamma_{\mathrm{t}}$ is raised from 5.42 to $6.60 . v_{\mathrm{x}}$ and $v_{\mathrm{y}}$ remain 6.61 and 8.61 respectively and the phase advance from pick-up to kicker in the core and stack-tail betatron systems remains close to 90 degrees. The natural chromaticities in each plane $\left(\xi_{x}=-11.54\right.$ and $\left.\xi_{y}=-13.42\right)$ are correctable by decreasing the current in one of the sextupoles in each sector. The aperture of the ring is $15 \pi \mathrm{mm}$-mrad unnormalized in both planes. The dispersion is similar to the present Accumulator with a slightly more negative dispersion in non-critical parts of the ring to accomplish the change in $\gamma_{t}$.

As the stack size increases the stacking efficiency falls. Figure 2.3.1 shows this dependence. The predicted decrease in stacking efficiency is due to three loss mechanisms which grow with stack size. First, a constant beam lifetime due to antiproton nuclear interaction with the residual vacuum molecules dictates that the instantaneous antiproton loss rate is proportional to the stack size. Eventually, the stack would grow to a point where the loss rate equals the gross stacking rate. At present the vacuum lifetime of the Accumulator is approximately 850 hours. Second, beam loss from both the stack-tail and the core portions of the stored beam due to the Accumulator momentum aperture and limitations in the stochastic stacking system. The expected evolution of the longitudinal density of the beam with increasing stack intensity is plotted in figure 2.3.2. Third, the transverse emittance of the antiprotons is expected to increase 
linearly as the stack intensity increases. Therefore, at some point the beam begins to start hitting the transverse aperture of the Accumulator. Figure 2.3.3 contains a plot of this expected dependence. Because of the growth of the transverse emittance with stack size, the luminosity per antiproton also diminishes as the stack size increases. Figure 2.3.4 shows the present operational experience with this effect.

Table 2.3.1: Summary of quadrupole gradient changes proposed to decrease the momentum compaction of the Accumulator lattice. The labels in the middle column are the standard Tevatron I nomenclature for the quadrupoles.

\begin{tabular}{|c|c|c|}
\hline $\begin{array}{c}\text { Quadrupole } \\
\text { Magnet }\end{array}$ & $\begin{array}{c}\text { TeV I } \\
\text { Design Field }\end{array}$ & $\begin{array}{c}\text { Upgrade Gradient/ } \\
\text { TeV I Gradient }\end{array}$ \\
\hline \hline Q1 & QT & 1.00 \\
\hline Q2 & -QT & .97 \\
\hline Q3 & QT & .87 \\
\hline Q4 & QSF & .97 \\
\hline Q5 & QSD & 1.00 \\
\hline Q6 & QSF & 1.10 \\
\hline Q7 & QSD & 1.00 \\
\hline Q8 & QSF & 1.00 \\
\hline Q9 & QSD & 1.00 \\
\hline Q10 & $0.45724 *$ LQ & 1.00 \\
\hline Q11 & LQ & 1.00 \\
\hline Q12 & - LQ & 1.01 \\
\hline Q13 & - LQ & 1.01 \\
\hline Q14 & LQ & 1.07 \\
\hline \hline QT Bus & $10.38087 \mathrm{~T}$ & .996 \\
\hline QSF Bus & $9.66333 \mathrm{~T}$ & .996 \\
\hline QSD Bus & $-9.74126 \mathrm{~T}$ & 1.005 \\
\hline LQ Bus & $8.93989 \mathrm{~T}$ & .998 \\
\hline
\end{tabular}

Antiproton extraction from the Accumulator core under the original Main Injector operational scenario would have been accomplished by adiabatically extracting fractions of the stored charge longitudinally by means of a frequency and amplitude ramped $h=4$ RF system. The Main Injector design report calls for $130 \times 10^{10}$ antiprotons at the beginning of stores. Assuming a $90 \%$ efficiency, a total of $140 \times 10^{10}$ antiprotons must be extracted. Because of the nature of RF unstacking, an initial stack intensity of $200 \times 10^{10}$ is required. For 36 bunch operation, 9 extraction cycles would be required. The Main Injector design antiproton emittance of $22 \pi \mathrm{mmmr}$ is based on these considerations. 


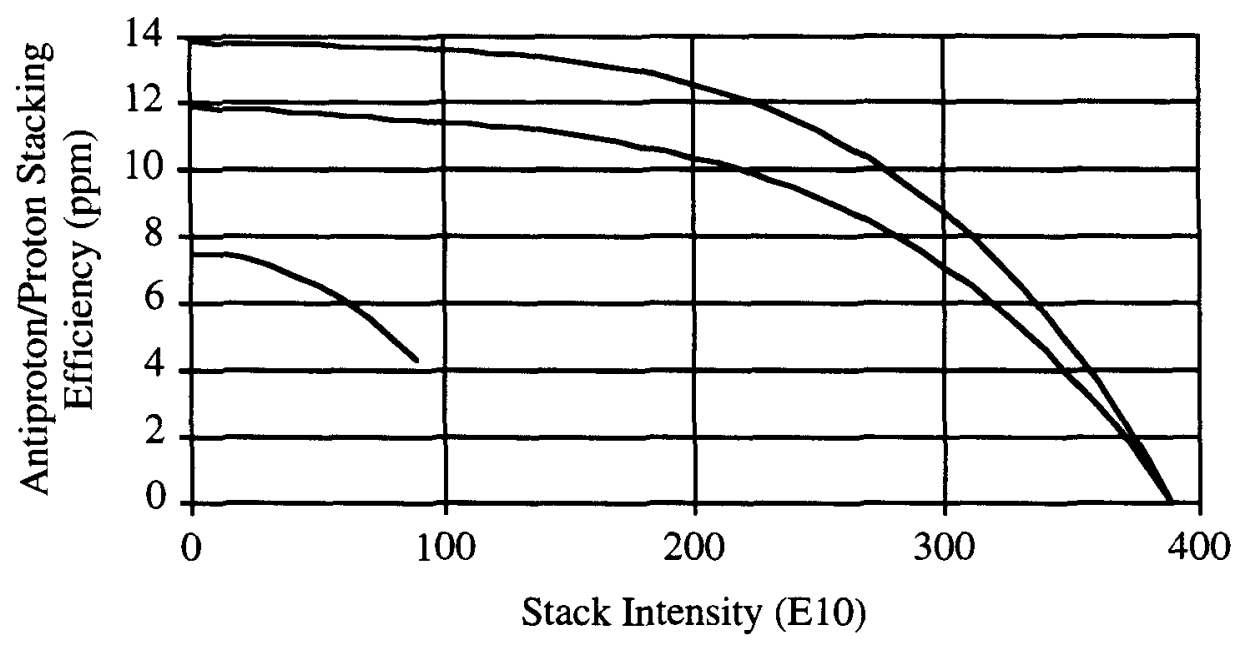

Figure 2.3.1: Stacking efficiency as a function of the antiproton stack size in the Accumulator. The lowest curve was measured during the 1988-9 collider run. The middle curve is the expected stacking profile if the stack-tail system remains in the $1-2 \mathrm{GHz}$ frequency band. Finally, the top curve is the prediction of the stacking rate if the $2-4 \mathrm{GHz}$ stack-tail stochastic cooling system is installed.

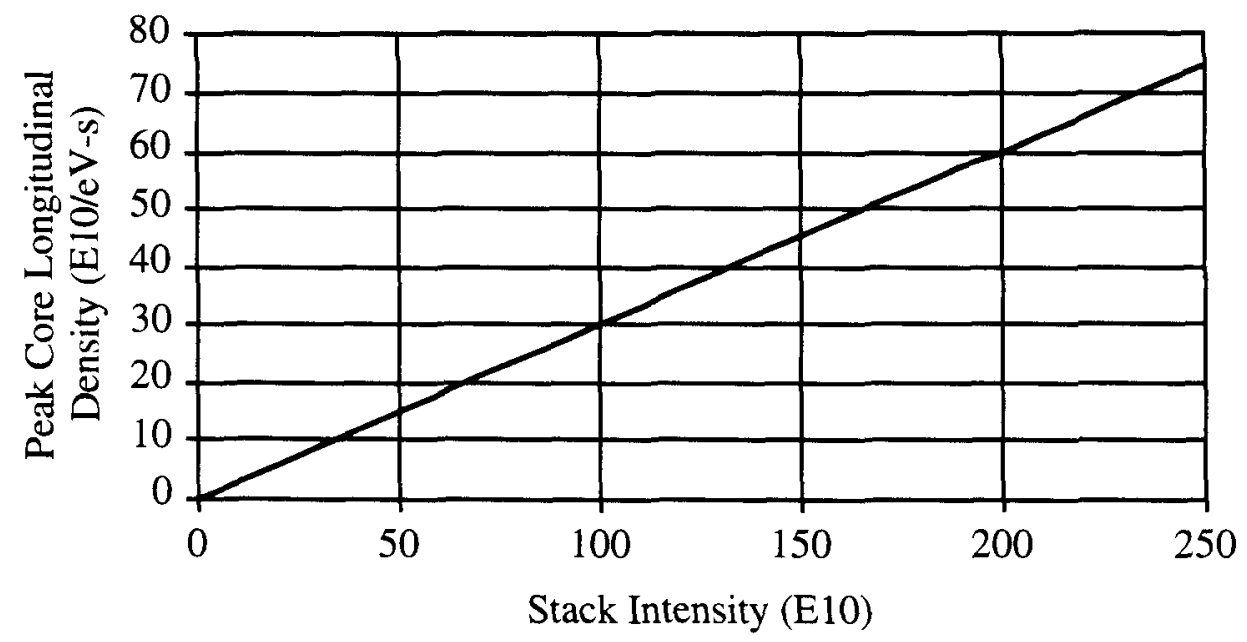

Figure 2.3.2: Dependence of the peak longitudinal density of the antiproton core as a function of the stack intensity anticipated after all upgrades associated with the Main Injector upgrade are completed. 


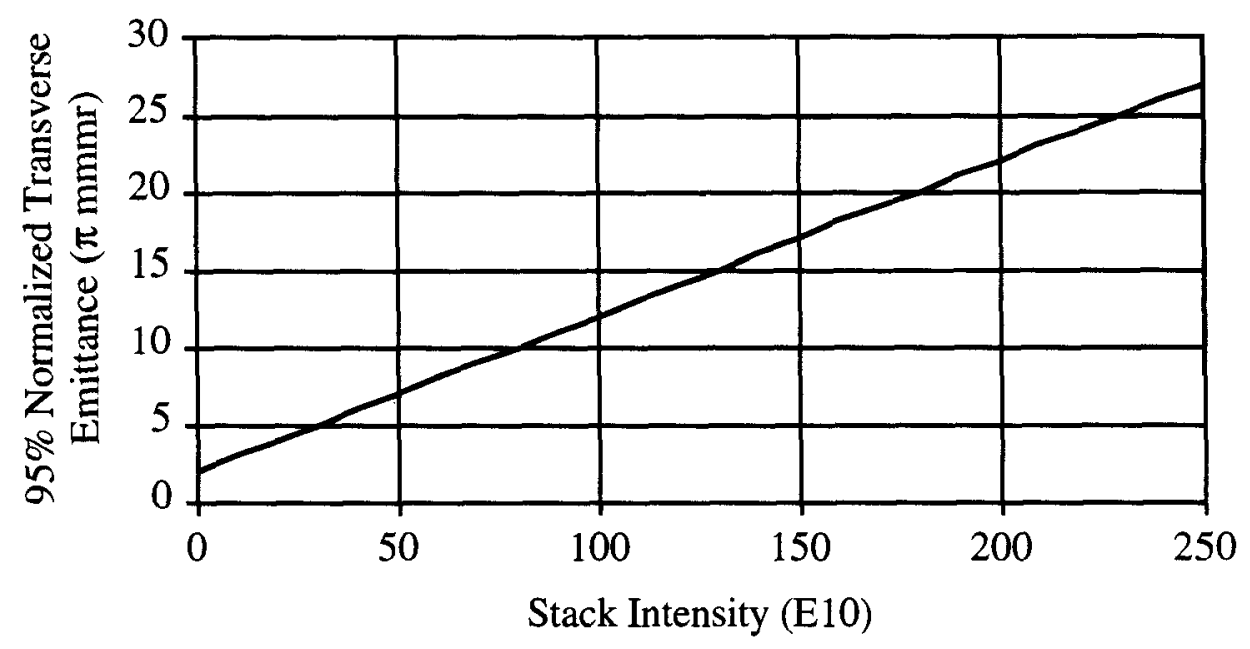

Figure 2.3.3: Prediction of the transverse emittance in the Accumulator as a function of the stack intensity.

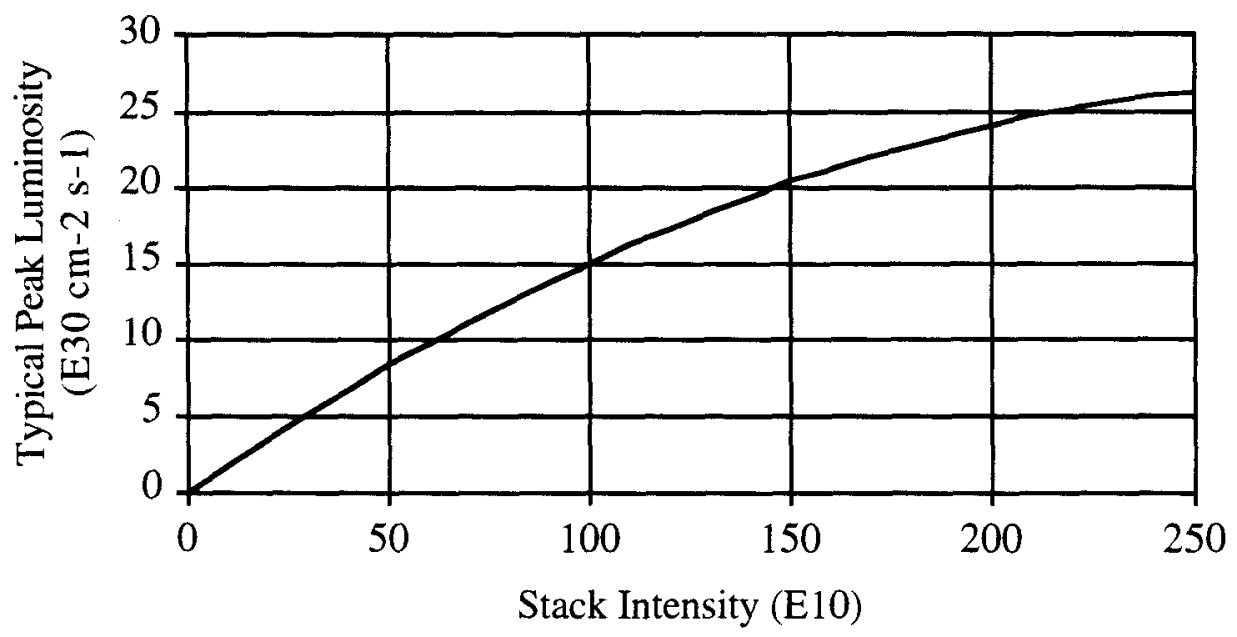

Figure 2.3.4: Observed luminosity vs. stack intensity curve for the present collider run. Instead of growing linearly, the luminosity saturates due to the increased transverse emittance of the antiproton stack.

\subsection{Recycler}

To maintain a high stacking rate in the Accumulator, the antiproton beam must be transferred to the Recycler while the Accumulator stack is not too large. The larger circumference and, more importantly, the presence of an electron cooling system in the Recycler, allow a much larger stack to be accumulated without significant loss of stacking rate. The time interval between transfers from the Accumulator to the Recycler is determined by three criteria. First, in order to keep the stack size small enough to stay at the optimum stacking rate, transfers should occur at least every roughly two hours. 
(See figure 2.3.1.) Second, to prevent the need for transfer line tune-up every time a transfer is attempted the time interval should be shorter than approximately 30 minutes. This latter interval is based on the observed reproducibility of antiproton transfers during long Tevatron Collider injection periods. Third, the electron cooling system in the Recycler must have enough time to cool the injected antiprotons so that the next transfer is possible.

\subsection{Symbol Definitions}

C Storage ring circumference

$\mathrm{D}(\mathrm{s})$ Horizontal dispersion function around the ring

$\eta \quad$ Momentum compaction factor

$\gamma_{\mathrm{r}} \quad$ Relativistic beam energy

$\gamma_{\mathrm{t}} \quad$ Relativistic transition crossing energy

$\rho(s) \quad$ Local radius of curvature around the ring 


\section{Antiproton Recycling}

The concept of retrieving antiprotons from the end of a collider store for use in the next store to increase initial luminosity is not controversial. The real issue is whether the amount of increase justifies the cost necessary to implement it. The three factors which will determine the efficacy of antiproton recycling are the percentage of the antiprotons left at the end of a store, the charge transfer efficiency from the Tevatron to the Recycler ring, and the time it takes to decelerate and reintegrate the antiprotons into the existing Recycler antiproton beam.

\subsection{Evolution of the Luminosity}

The evolution of the luminosity and antiproton intensity during a Tevatron Collider store is determined by the luminosity itself, intrabeam scattering, external transverse and longitudinal emittance growth mechanisms, and the length of the store. In addition, the reliability of the Tevatron must be great enough to ensure that the end of most stores is reached with the antiprotons intact.

The percentage of stores ended prematurely by some type of equipment or power failure has been tracked over the years. At the beginning of collider runs this percentage starts out higher as weak components are shaken out, but in general the value of $10 \%$ is typical, or a little over 1 store per week. For the sake of later calculations, let the symbol $\mathrm{P}_{\mathrm{S}}$ represent the fraction of stores in which antiproton recycling is possible.

The luminosity $\mathrm{L}$ is determined by the equation

$$
L=\frac{N_{P}\left(N_{A} B\right) f_{o}\left(6 \beta_{r} \gamma_{r}\right)}{2 \pi \beta^{*}\left(\varepsilon_{n_{P}}+\varepsilon_{n_{A}}\right)} H\left(\frac{\beta^{*}}{\sigma_{s}}\right) \frac{1}{\sqrt{1+\frac{2 \alpha^{2} \sigma_{s}^{2}\left(6 \beta_{r} \gamma_{r}\right)}{\beta^{*}\left(\varepsilon_{n_{P}}+\varepsilon_{n_{A}}\right)}}}
$$

in which $N_{p, a}$ is the number of protons and antiprotons per bunch, $B$ is the number of bunches per beam, $f_{o}$ is the revolution frequency of the Tevatron, $\beta_{r}$ and $\gamma_{r}$ are the relativistic velocity and energy of the beam, $\beta^{*}$ is the value of the beta function at the interaction points, $\varepsilon_{\mathrm{np}, \mathrm{na}}$ are the proton and antiproton $95 \%$ normalized transverse emittances, $\sigma_{\mathrm{s}}$ is the rms bunch length, $\alpha$ is the crossing half angle between the beams at the interaction points, and the hour-glass luminosity form factor has the form

$$
H(x)=\sqrt{\pi} x[1-\Phi(x)] \mathrm{e}^{x^{2}}
$$

The transverse emittance is defined so that the transverse rms bunch widths are calculated using the equation

$$
\sigma_{\mathrm{a}, \mathrm{p}}^{2}=\beta^{*} \frac{\varepsilon_{\mathrm{na}, \mathrm{np}}}{\left(6 \beta_{\mathrm{r}} \gamma_{\mathrm{r}}\right)}
$$

The values for all of these parameters at the beginning of a store for present operations, anticipated after completion of the original Main Injector project, and after commissioning of the Recycler ring (Tevatron* column) are listed in Table 3.1.1. 
Table 3.1.1: Initial Beam conditions for the present run, originally anticipated post-Main Injector run, and in the era of Tevatron* in which the Recycler ring is operational.

\begin{tabular}{|c|c|c|c|}
\hline Initial Store Parameters & Present & $\mathrm{MI}$ & Tev $^{*}$ \\
\hline $\mathrm{N}_{\mathrm{p}}$ Proton Intensity/Bunch $\left(10^{10}\right)$ & 25 & 38 & 27 \\
\hline $\mathrm{N}_{\mathrm{a}}$ Antiproton Intensity/Bunch $\left(10^{10}\right)$ & 8 & 3.6 & 5.5 \\
\hline B Number of Bunches/Beam & 6 & 36 & 36 \\
\hline Minimum Time between Bunches (ns) & 3500 & 395 & $395(132)$ \\
\hline Eo Beam Energy (GeV) & 900 & 1000 & 1000 \\
\hline$\beta^{*}$ Interaction Point Beta $(\mathrm{cm})$ & 35 & 35 & 35 \\
\hline$\varepsilon_{\mathrm{np}}$ Proton $95 \%$ Emittance $(\pi \mathrm{mmmr})$ & 24 & 30 & 18 \\
\hline$\varepsilon_{\text {na }}$ Antiproton $95 \%$ Emittance $(\pi \mathrm{mmmr})$ & 15 & 15 & 15 \\
\hline$\sigma_{\mathrm{s}} \quad$ Rms Bunch Length (cm) & 50 & 45 & 20 \\
\hline fo Revolution Frequency $(\mathrm{kHz})$ & 47.7 & 47.7 & 47.7 \\
\hline$\alpha \quad$ Crossing Half Angle (mr) & 0 & 0 & 0 \\
\hline$\left(\mathrm{N}_{2} \mathrm{~B}\right)$ Total Antiproton Intensity $\left(10^{10}\right)$ & 48 & 130 & 200 \\
\hline$(\beta \mathrm{r} \gamma \mathrm{r})$ Relativistic Momentum & 959 & 1066 & 1066 \\
\hline $\mathrm{H} \quad$ Hour Glass Form Factor & 0.65 & 0.69 & 0.88 \\
\hline Crossing Angle Form Factor & 1.00 & 1.00 & 1.00 \\
\hline Peak Luminosity $\left(10^{32} \mathrm{~cm}^{-2} \mathrm{sec}^{-1}\right)$ & 0.25 & 1.0 & 2.0 \\
\hline Integrated Luminosity ( $\mathrm{pb}^{-1 / \text { week })}$ & 5.0 & 21 & 40 \\
\hline
\end{tabular}

The evolution of the above parameters during stores determines the number of antiprotons remaining at the end. The parameters which depends on luminosity itself are the proton and antiproton intensity lifetimes. The central purpose of the Tevatron Collider is to collide protons and antiprotons, using them up at the rate of

$$
\mathrm{R}_{\text {lost }}=\mathrm{N}_{\mathrm{IR}} \mathrm{L} \sigma_{\text {lost }}
$$

where $\sigma_{\text {lost }}$ is the $78 \mathrm{mb}$ cross-section for losing protons and antiprotons due to both inelastic and large elastic interactions and $\mathrm{N}_{I R}$ is the number of interaction regions in the Collider. The proton and antiproton intensity lifetimes due to this mechanism are calculated using the equation

$$
\tau_{\mathrm{Lp}, \mathrm{La}}=\frac{\mathrm{N}_{\mathrm{p}, \mathrm{a}} \mathrm{B}}{\mathrm{R}_{\text {lost }}}
$$

Another limit to beam intensity lifetime is the residual vacuum in the Tevatron. The vacuum intensity lifetime $\tau_{\mathrm{vac}}$ is approximately 200 hours at the present time.

The other parameters which vary during a store are the bunch length and the transverse emittance. The first mechanism observed to induce emittance growth during a store is external noise. When noise driven coherent betatron and synchrotron oscillations decohere due to nonlinearities, a constant emittance growth rate is established. Over the years this emittance growth rate has been lowered to a fairly insignificant level of 
$0.3 \pi \mathrm{mmmr} / \mathrm{hr} \mathrm{rms}$ normalized transversely $\left(\mathrm{g}_{\mathrm{T}}\right)$ and $0.01 \mathrm{eV}$-sec/hr $95 \%$ normalized longitudinally $\left(\mathrm{g}_{\mathrm{L}}\right)$. The second mechanism is elastic scattering against the residual molecules in the vacuum chamber.

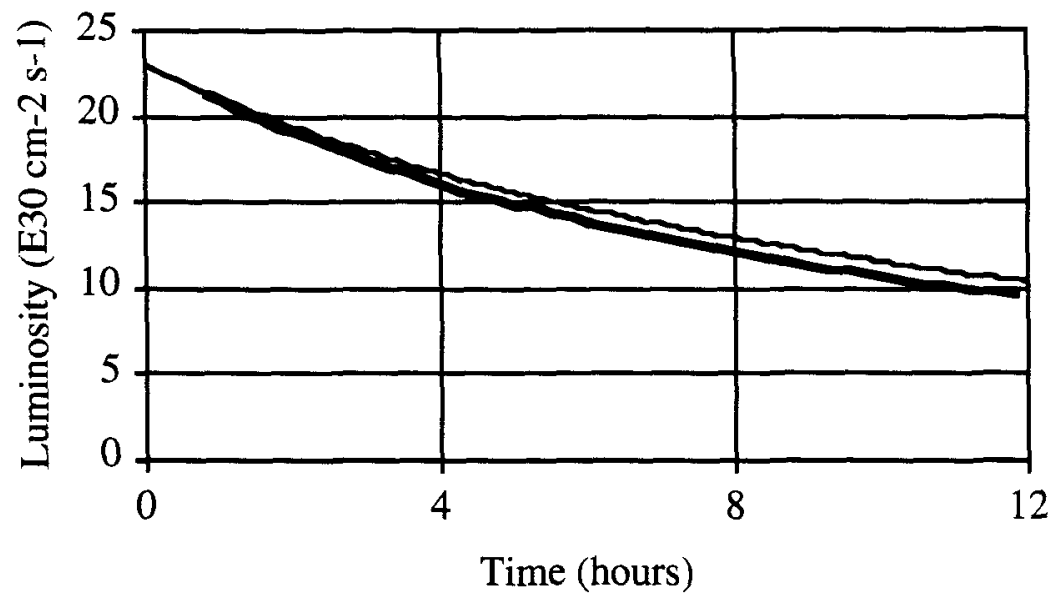

Figure 3.1.1: Prediction (thin) and measurements (thick) of collider luminosity as a function of time during a recent Tevatron Collider store. The relative luminosity was adjusted to account for a known systematic calibration error in the measured data.

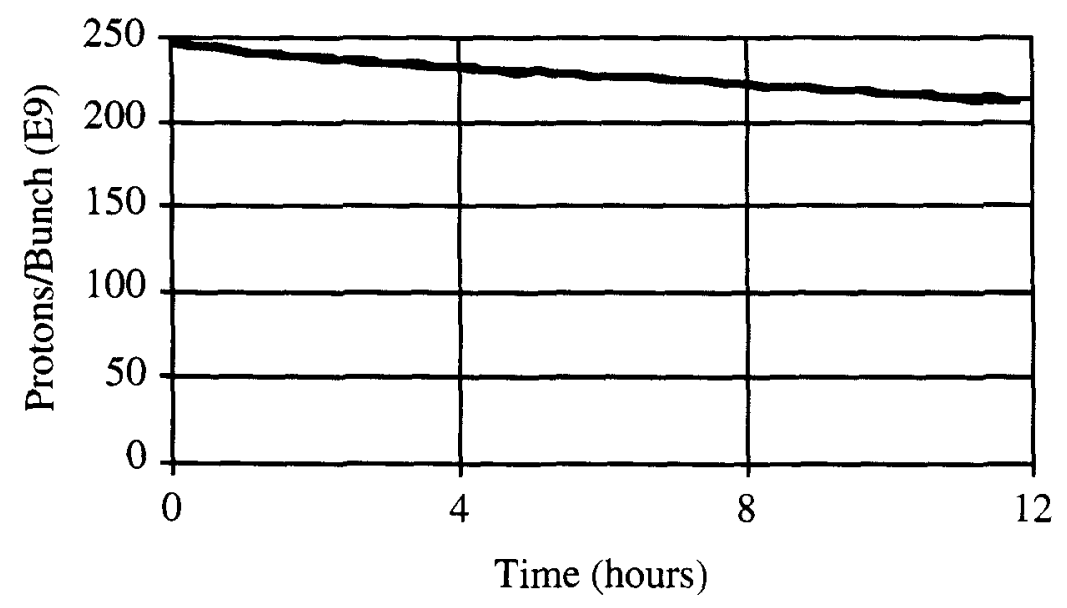

Figure 3.1.2: Prediction (thin) and measurements (thick) of the intensity per proton bunch vs. time during a recent store.

The growth times of the transverse and longitudinal emittances due to intrabeam scattering are described by the equations [D. Finley, TM-1646 (1989)]

$$
\tau_{\varepsilon_{p}}=0.054\left(\frac{6 \times 10^{10}}{N_{P}}\right) \varepsilon_{n_{P}}^{2.24} A_{P}^{0.68}
$$




$$
\tau_{\mathrm{Ap}}=0.103\left(\frac{6 \times 10^{10}}{\mathrm{~N}_{\mathrm{P}}}\right) \varepsilon_{\mathrm{n}_{\mathrm{P}}}^{1.24} \mathrm{~A}_{\mathrm{P}}^{1.68}
$$

where $\varepsilon_{\mathrm{nP}}$ and $\mathrm{AP}_{\mathrm{P}}$ are the normalized $95 \%$ transverse and longitudinal emittances of the protons and the times are in hours. The antiprotons growth times are calculated similarly.

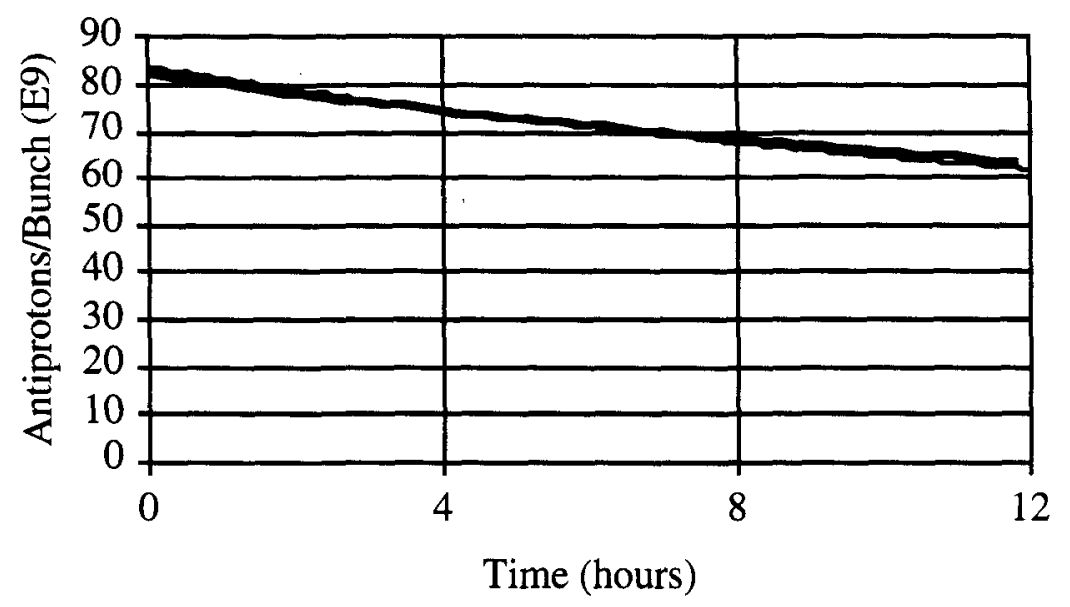

Figure 3.1.3: Prediction (thin) and measurements (thick) of the intensity per antiproton bunch as a function of time during a recent Tevatron Collider store.

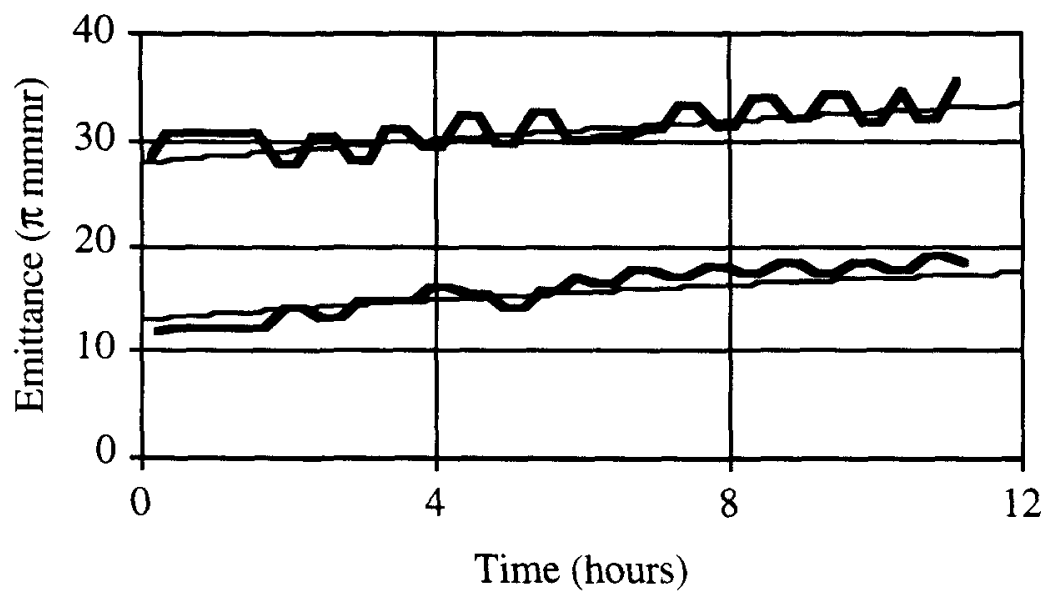

Figure 3.1.4: Prediction (line) and measurements (squares) of the proton (upper) and antiproton (lower) transverse $95 \%$ invariant emittances as a function of time during a recent Tevatron Collider store.

By including all of the above effects into a calculation of the evolution of the luminosity and beam properties during a collider beam store, predictions can be made. In order to confirm that the factors affecting luminosity evolution are understood, these calculations were applied to the present collider run. Figure 3.1.1 shows the predicted and measured luminosity evolution during a high luminosity store. The intensity 
decrease of the beams during the store are shown in figures 3.1.2 and 3.1.3. The transverse and longitudinal 95\% invariant emittances are displayed in figures 3.1.4 and 3.1.5. In order to verify that these predictions are not "tuned" to the data, a low antiproton intensity store was measured, and the same calculation was used to predict the variation of the beam parameters in that store. The largest difference with the store shown in figures 3.1.1 to 3.1.5 was in the longitudinal emittance. The evolution of the longitudinal proton and antiproton $95 \%$ invariant emittances still agreed with the calculation. This model is quantitatively in agreement in all beam parameters in all measured stores in which comparisons have been performed. Therefore, it is with confidence that predictions of Tevatron* performance are presented in this chapter.

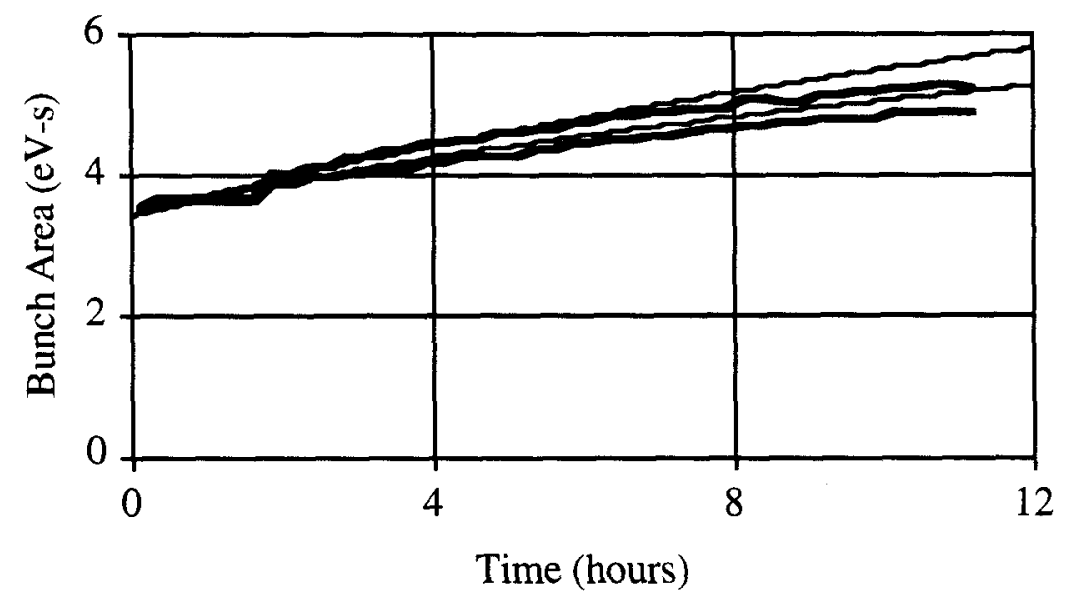

Figure 3.1.5: Prediction (thin) and measurements (thick) of the proton (upper) and antiproton (lower) longitudinal 95\% invariant emittances as a function of time during a recent Tevatron Collider store.

The prediction of Tevatron* luminosity evolution appears in figure 3.1.6. The luminosity drops by a factor of two in about four hours. The next factor of two drop requires more than 8 hours. The reason for this increase in the luminosity lifetime with elapsed time in the store is intrabeam scattering. The transverse emittance time evolution of both the protons and antiprotons are shown in figure 3.1.7. The emittance growth rates are initially steep and decrease with time in the store. These growth rates are caused by the intrabeam scattering contribution to the model, verifying the above assertion. In addition, the longitudinal emittance of the protons and antiprotons also grow during a store due to intrabeam scattering. The predictions for this effect of intrabeam scattering are displayed in figure 3.1.8.

The longitudinal emittances of the proton and antiproton bunches start out quite small due to the fact that the Recycler ring is capable of forming the required intensity bunches without the use of coalescing. The 11 proton and antiproton bunches before coalescing in the present collider run have a longitudinal emittance of approximately $0.2 \mathrm{eV}$-s each. Instead of the expected longitudinal emittance of $2.2 \mathrm{eV}$-s for the coalesced bunch, the initial emittance ends up as more than $3.5 \mathrm{eV}$-s. The process of coalescing dilutes the longitudinal emittance by more than $50 \%$. 


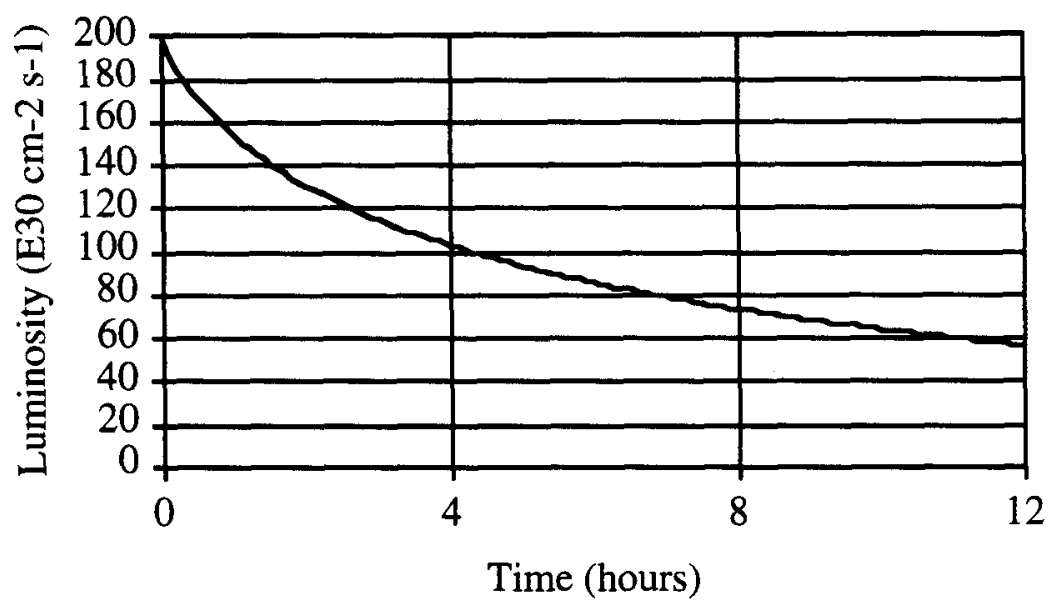

Figure 3.1.6: Prediction of the time evolution of luminosity during a Tevatron Collider store after the Tevatron* upgrade.

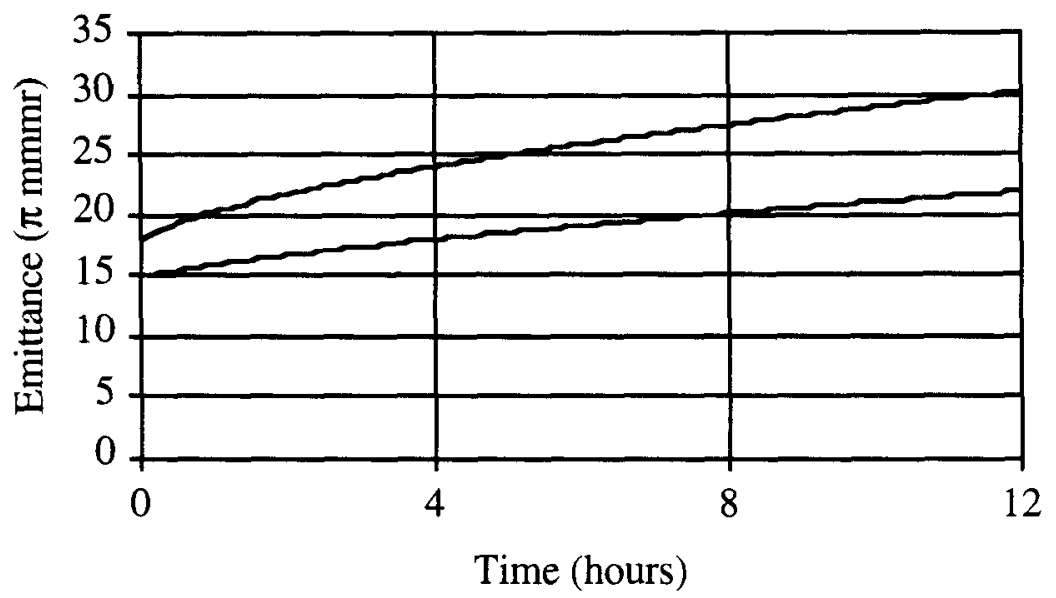

Figure 3.1.7: Predictions of the proton (upper) and antiproton (lower) transverse $95 \%$ invariant emittances as a function of time during Tevatron* operations.

Below transition the longitudinal emittance of each proton bunch is approximately $0.05 \mathrm{eV}-\mathrm{s}$, where the dilution to $0.2 \mathrm{eV}-\mathrm{s}$ is due to transition crossing. With Booster bunch intensities of $60 \times 10^{9}$, the required collider proton bunch of $270 \times 10^{9}$ needs the charge from 5 Booster bunches. By debunching in the Recycler ring, merging the charge adiabatically with electron cooling and a low voltage RF system, initial collider bunch longitudinal emittances of $0.3 \mathrm{eV}$-s or lower are possible. These bunches are accelerated in the Main Injector through transition and transferred into the Tevatron. The Main Injector has a longitudinal admittance of $0.5 \mathrm{eV}$-s. Even assuming dilution from transition crossing and transfer into the Tevatron, longitudinal emittances below $0.6 \mathrm{eV}-\mathrm{s}$ are very probable. 


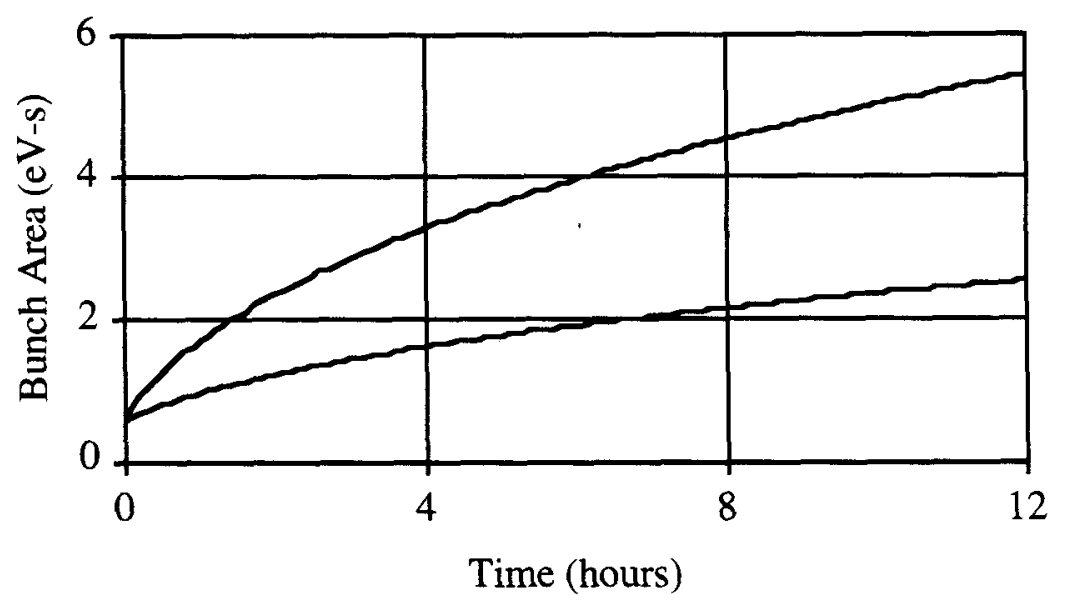

Figure 3.1.8: Predictions of the proton (upper) and antiproton (lower) longitudinal $95 \%$ invariant emittances as a function of time during Tevatron* operations.

For $36 \times 36$ bunch operation, the total longitudinal emittance of the antiprotons stored in the Recycler ring must be partitioned into 36 equal portions. The total invariant $95 \%$ longitudinal emittance of the Recycler ring $A_{r}$ is

$$
A_{\mathrm{r}}=4 \mathrm{~T}_{\mathrm{o}} \sigma_{\mathrm{e}}
$$

Given the Main Injector longitudinal admittance of $0.5 \mathrm{eV}-\mathrm{s}$, the total Recycler longitudinal emittance must be less than $20 \mathrm{eV}$-s, or the partitioned charge must be electron cooled after being partially bunched to attain the $0.5 \mathrm{eV}$-s limit. For reference, the $20 \mathrm{eV}$-s emittance corresponds to an rms energy spread of $450 \mathrm{keV}$, or a fractional energy spread of approximately $5 \times 10^{-5}$.

In the Accumulator, a longitudinal emittance of $6.4 \mathrm{eV}-\mathrm{s}$ is produced by the longitudinal stochastic cooling system, independent of the antiproton stack size. This corresponds to an rms energy spread of $1 \mathrm{MeV}$. For a ring approximately $1 / 7$ th the circumference of the Recycler, this corresponds to half the phase space density found in the $20 \mathrm{eV}$-s Recycler scenario. Since the electron cooling time is anticipated to be significantly shorter that of the Accumulator cooling system, the value of $20 \mathrm{eV}-\mathrm{s}$ is achievable. Nonetheless, calculations of microwave instability and intrabeam scattering are required to confirm that this level of global cooling can be performed, and how much cooling of the partitioned charge is required.

In the case of $108 \times 108$ bunch operation, which corresponds to a minimum bunch spacing of $132 \mathrm{~ns}$, a total longitudinal emittance of $55 \mathrm{eV}$-s is acceptable. The global fractional energy spread of $10^{-4}$ is much less demanding than that required in the Accumulator. 


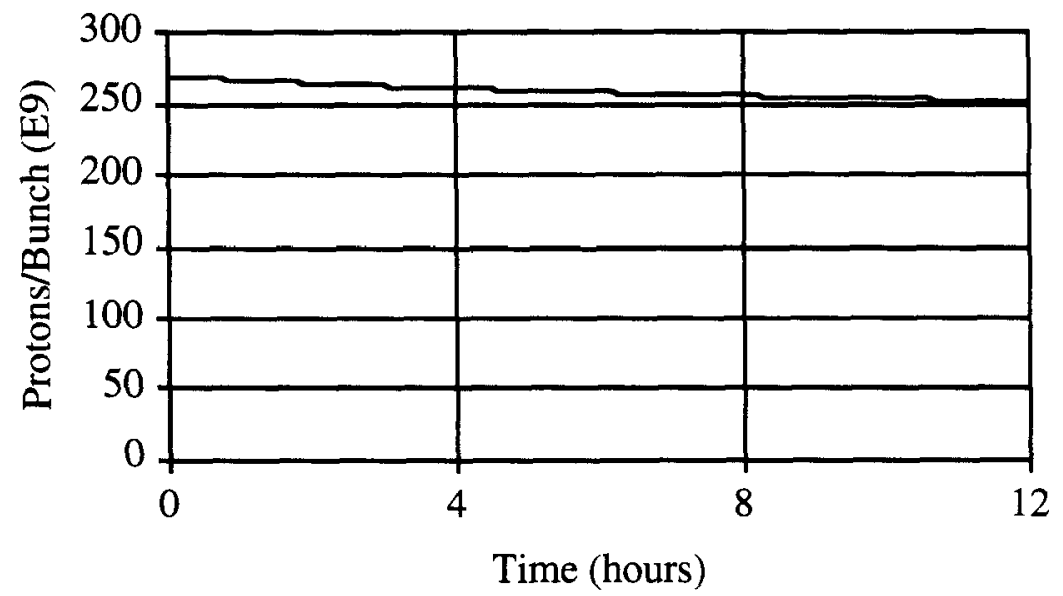

Figure 3.1.9: Prediction of the proton bunch intensity as a function of time during Tevatron* operations.

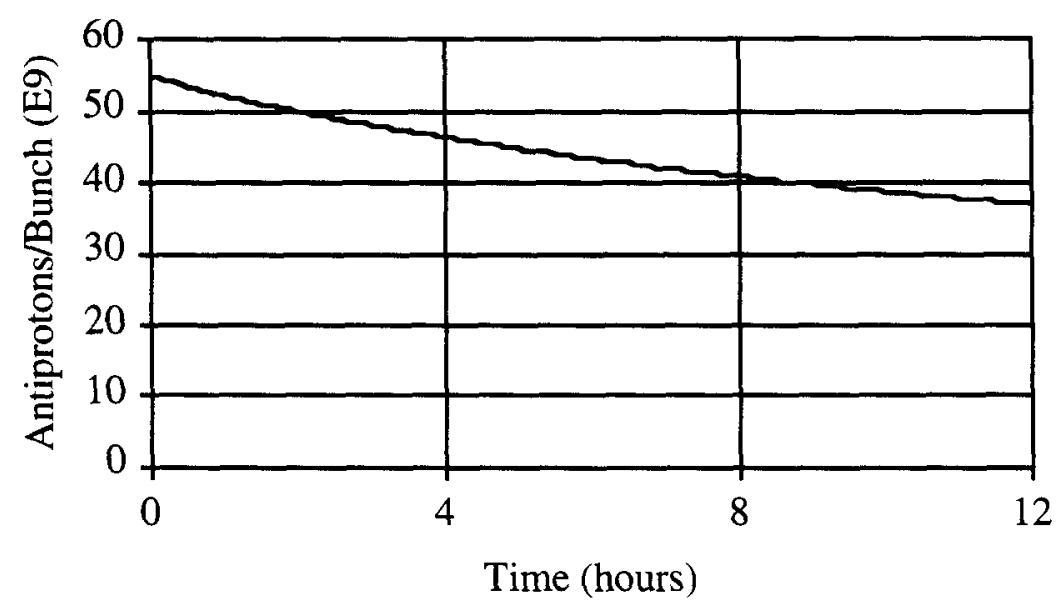

Figure 3.1.10: Prediction of the antiproton bunch intensity as a function of time during Tevatron* operations.

The time evolution of the proton and antiproton bunch intensities are shown in figures 3.1.9 and 3.1.10. As expected, because the rate of particle loss for both beams is equal but the antiproton total intensity is much lower than that of the protons, the proton intensity lifetime is much longer than the antiproton lifetime. In order to assess the benefit of antiproton recycling, the percentage of the original antiproton intensity remaining at the end of the store is critical. The next task is to consider the optimum length of a store.

\subsection{Optimum Store Length}

The goal of the Tevatron Collider is to integrate luminosity at the highest rate possible. In order to achieve this goal, high initial luminosities, long luminosity lifetimes, and high stacking rates are required. The store length $T_{\mathrm{S}}$ is also equal to the time 
available for stacking between injections. The filling time $T_{f}$ is determined by a number of factors not relevant to this discussion. In present operations using the Accumulator to stack antiprotons the time evolution of the luminosity and antiproton stack size have the dependencies sketched in figure 3.2.1. The total Tevatron Collider cycle time $T_{\mathcal{C}}$ is the sum of the store time and the fill time.

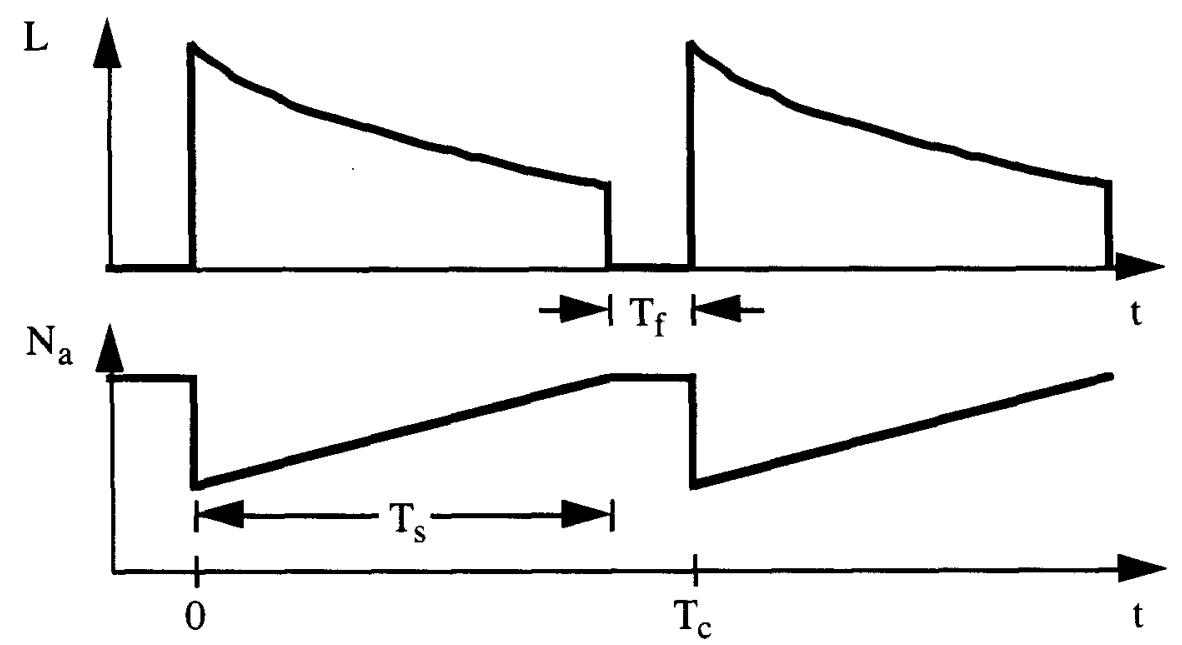

Figure 3.2.1: Sketch of the time dependence of luminosity and accumulated antiproton stack intensity. The store time $\mathrm{T}_{\mathrm{s}}$ plus the fill time $\mathrm{T}_{\mathrm{f}}$ equals the total collider cycle time of $\mathrm{T}_{\mathrm{c}}$.

To maximize the rate at which integrated luminosity is delivered, it is necessary to maximize the average luminosity $\langle\mathrm{L}\rangle$, which is defined as

$$
\langle L\rangle=\frac{1}{T_{s}+T_{f}} \int_{0}^{T_{s}} L(t) d t
$$

The model used to predict the luminosity and other beam parameters during Tevatron* operations also generates the integrated luminosity vs. time during a store. By allowing the store time to be the independent variable, the dependence of the average luminosity on store duration for a number of choices for the fill time is plotted in figure 3.2.2. As expected, shorter fill times correspond to high average luminosities and shorter optimum store lengths. Even though fill times of one half hour are possible, it is more likely that fill times around 1 hour will be operationally feasible. At this level, there is only a $10 \%$ difference in average luminosity between the optimum of 3 hours and 6 hours.

The main impact of the store length is the antiproton stacking rate required to maintain the design luminosity of Tevatron*. In order to understand how the curve in figure 3.2.3 describing the required stacking rate vs. store duration was generated, refer to table 3.2.1. 


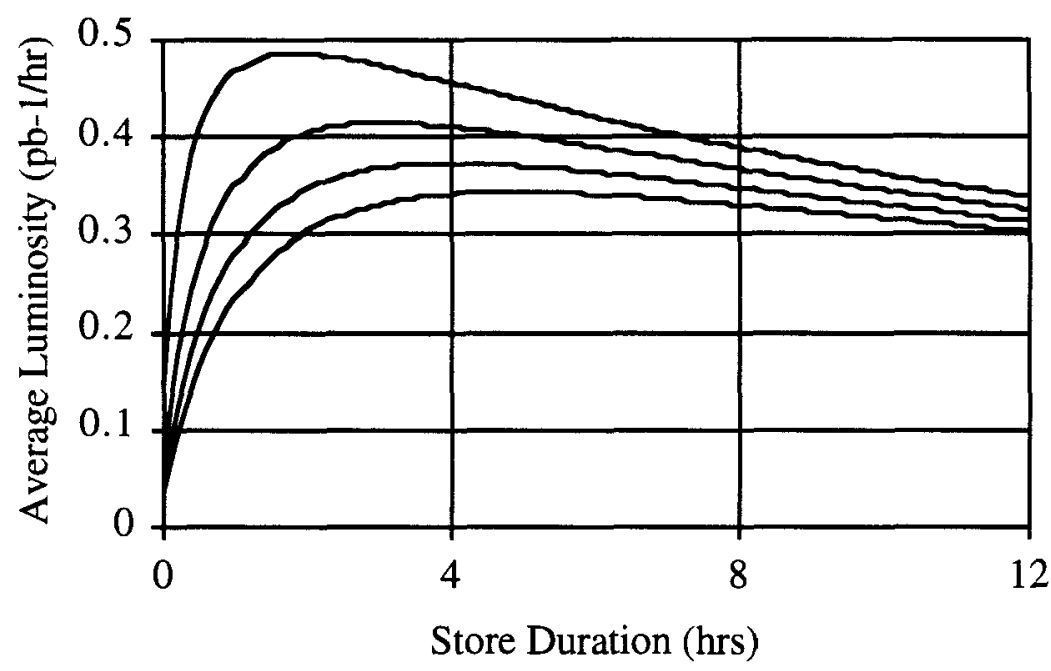

Figure 3.2.2: Prediction of the average luminosity vs. store duration for fill times of 0.5 (top), 1.0, 1.5, and 2.0 (bottom) hours. The longer the fill time, the longer is the optimum store duration.

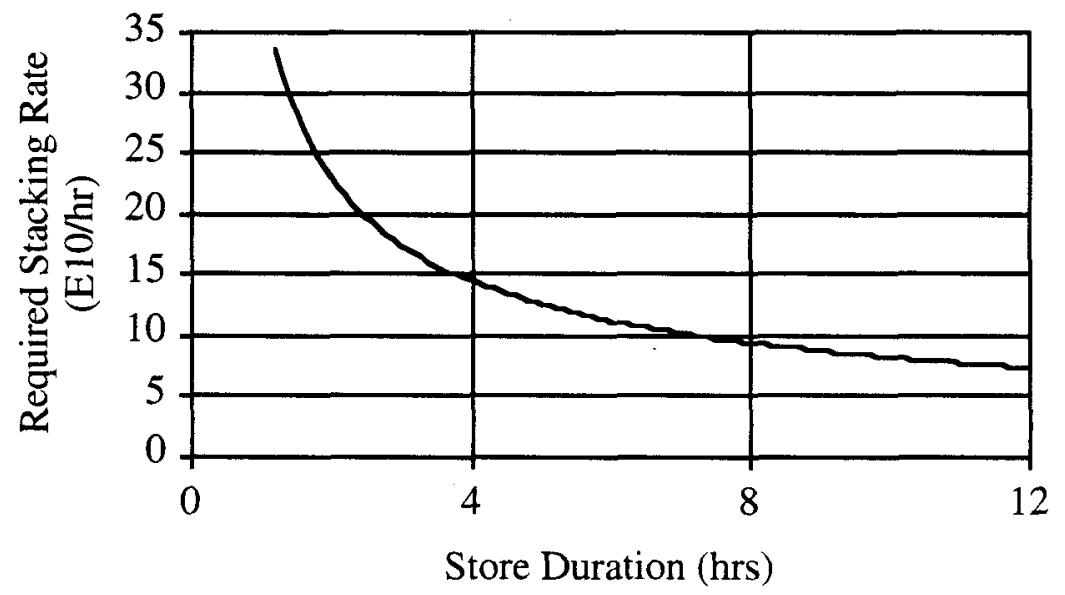

Figure 3.2.3: Prediction of the stacking rate required to achieve repeated stores at the peak luminosity of $2 \times 10^{32} \mathrm{~cm}^{-2} \mathrm{~s}^{-1}$.

The values in the rows labeled "Antiprotons at End of Store" and "Integrated Luminosity" were generated by the luminosity evolution calculations described above. The fill time for the MI and Tevatron* are estimates. The acceleration efficiency is less than the value of $95 \%$ chosen in the original Main Injector design. The deceleration efficiency is basically the acceleration efficiency plus a factor due to the process of decoalescing. Decoalescing is the process by which the $1.5 \mathrm{eV}$-s collider antiproton bunches are broken up into smaller individual bunches so as to cross back down through transition without hitting the longitudinal admittance at transition. Plugging in all of these parameter values, one can generate the subsequent numbers. 
Table 3.2.1: Parameters which describe the effect of recycling antiprotons on antiproton stacking and average luminosity.

\begin{tabular}{|l|c|c|c|}
\hline Parameter & Present & MI & Tev $^{*}$ \\
\hline \hline Store Duration $\mathrm{T}_{\mathrm{S}}(\mathrm{hr})$ & 12 & 12 & 6 \\
\hline Injection Time $\mathrm{T}_{\mathrm{f}}(\mathrm{hr})$ & 2.5 & 2 & 1 \\
\hline Antiprotons at End of Store & $73 \%$ & $65 \%$ & $79 \%$ \\
\hline Deceleration Efficiency & $0 \%$ & $0 \%$ & $80 \%$ \\
\hline Acceleration Efficiency & $75 \%$ & $90 \%$ & $90 \%$ \\
\hline Integrated Luminosity $\left(\mathrm{pb}^{-1}\right)$ & 0.74 & 2.9 & 1.2 \\
\hline Required Usable Stack $\left(10^{10}\right)$ & 64 & 144 & 220 \\
\hline Antiprotons Recycled $\left(10^{10}\right)$ & 0 & 0 & 125 \\
\hline New Antiprotons Stacked $\left(10^{10}\right)$ & 64 & 144 & 95 \\
\hline Required Stacking Rate $\left(10^{10} / \mathrm{hr}\right)$ & 5.3 & 12 & 15.8 \\
\hline Average Luminosity (pb-1/hr) & 0.05 & 0.21 & 0.39 \\
\hline $\begin{array}{l}\text { Store Hours Needed to Achieve the } \\
\text { Snowmass Criterion Between } \\
\text { Integrated and Peak Luminosity }\end{array}$ & 98 & 101 & 102 \\
\hline
\end{tabular}

The required usable stack is simply the initial total antiproton intensity in the Tevatron divided by the acceleration efficiency. In other words, it the number of antiprotons which must be extracted from the Accumulator now or the Recycler in the future in order to attain the number of antiprotons called for in table 3.1.1. The number of antiprotons recycled is the initial antiproton intensity times the fraction of antiprotons remaining at the end of the store times the deceleration efficiency. The number of antiprotons stacked is simply the difference between the required stack size and the number of antiprotons recycled. In the case of Tevatron*, recycling contributes more than a factor of two to the luminosity. The stacking rate is just the number of stacked antiprotons divided by the store duration.

The average luminosity is calculated using equation 3.2.1 and the parameter values for the store duration, fill time, and integrated luminosity per store. In tables such as 3.1.1 there is typically a row which contains the predicted integrated luminosity per week or month. This number is usually the integrated luminosity assuming the store is always at the peak luminosity the entire time period, and then derated by a factor of 3 . This is commonly referred to as the Snowmass criterion. Given the average luminosity, a certain number of store hours in a week are thus required in order to achieve the Snowmass criterion. In the case of Tevatron* this number is approximately the same as present operations.

The stacking rate varies with store length mainly because of the acceleration and deceleration efficiencies. As the store length is reduced, antiprotons are recirculated so often that the dominant mechanism for loss is the transfers. On the other hand, if the stores are kept too long, it is easy to achieve the peak luminosity goal but the number of store hours per week must increase. This trade-off is demonstrated in table 3.2 .2 and figure 3.2.3. 
Table 3.2.2: Effect of Tevatron* store length on the required stacking rate and store hours required to meet the Snowmass expectation of integrated luminosity per week.

\begin{tabular}{|l|c|c|c|}
\hline Parameter & 2 Hours & 4 Hours & 8 Hours \\
\hline \hline Required Stacking Rate $\left(10^{10} / \mathrm{hr}\right)$ & 38 & 22 & 13 \\
\hline $\begin{array}{l}\text { Store Hours Needed to Achieve the } \\
\text { Snowmass Criterion Between } \\
\text { Integrated and Peak Luminosity }\end{array}$ & 98 & 96 & 108 \\
\hline
\end{tabular}

\subsection{Tevatron Requirements}

In order to achieve the luminosity goal of Tevatron*, the proton and antiproton beams will have certain properties at injection and extraction from the Tevatron. This section is a review of the beam parameter values and issues associated with the transfers.

Because both protons and antiprotons will be cooled by the Recycler, it is imperative that the antiprotons are injected into the Tevatron first, emptying the Recycler. The 36 antiproton bunches will be transferred in 3 trains of 12 bunches separated by 21 buckets of $53 \mathrm{MHz}$ RF. Because of the admittance of the Main Injector, the $95 \%$ invariant longitudinal emittance of the bunches will be $0.5 \mathrm{eV}$-s or less. The intensity per bunch will be slightly larger than $8 \times 10^{10}$ /bunch. The protons must be transferred in batches of 4 bunches in order to fit their injection into the abort gap spacing between the threefold symmetric antiproton batches. The initial intensity and longitudinal emittance per bunch is anticipated to be approximately $28 \times 10^{10}$ and $0.5 \mathrm{eV}$-s respectively. For both beams the transverse $95 \%$ normalized emittances are expected to be less than $20 \pi \mathrm{mmmr}$.

At the end of the store the protons will be scraped away. In order to speed up this task and make it more reliable, it should be automated. In addition, a special thick collimator with warm Lambertson magnets or other massive beamline elements just downstream should be installed to reduce the risk of quenches. At the end of 6 hours the transverse and longitudinal emittances of the antiproton bunches are $18 \pi \mathrm{mmmr}$ and $2 \mathrm{eV}$-s. Both of these beam dimensions are smaller than bunches presently injected into the Tevatron. The transfer lines between the Main Injector and Tevatron were designed to cleanly transfer beams larger than this.

The one new step required in the Tevatron is the deceleration of the antiprotons. Because of the architecture of the CAMAC 465 control cards which control most RF waveforms and corrector power supplies, the end of store distributed TCLK event $\$ 46$ will initiate power supply, corrector, and RF ramps especially designated for this operation. These tables can be tuned up by decelerating protons from $900 \mathrm{GeV}$.

\subsection{Main Injector Requirements}

In order to transfer the antiprotons into the Tevatron first, the upgraded fast risetime Tevatron injection kicker originally built as an antiproton kicker must be moved to the proton injection line. A benefit of this change is that the maximum average intensity in the Main Injector is reduced during fills.

Transition crossing in the Main Injector is the dominant reason for anticipated longitudinal emittance dilution and beam loss in the acceleration process of the proton 
bunches. It is not known at this time whether bunches with $28 \times 10^{10}$ protons/bunch at a longitudinal emittance of $0.5 \mathrm{eV}$-s or smaller are stable during transition crossing.

As in the Tevatron, the 36 antiproton bunches will be transferred in 3 trains of 12 bunches separated by 21 buckets of $53 \mathrm{MHz}$ RF. Because of the admittance of the Main Injector, the $95 \%$ invariant longitudinal emittance of the bunches will be $0.5 \mathrm{eV}$-s or less. The intensity per antiproton bunch will be slightly larger than $8 \times 10^{10} / \mathrm{bunch}$. The protons must be transferred in batches of 4 bunches in order to fit their injection into the abort gap spacing between the threefold symmetric antiproton batches. The initial proton intensity per bunch is anticipated to be approximately $28 \times 10^{10}$. For both beams the transverse 95\% normalized emittances are expected to be less than $20 \pi \mathrm{mmmr}$, starting out near the present value of $10 \pi \mathrm{mmmr}$ at injection.

In order to achieve deceleration of the antiprotons, the collider bunches must be decoalesced into a number of smaller bunches in order to fit into the longitudinal admittance of the Main Injector at transition crossing. Therefore, at $150 \mathrm{GeV}$ the antiprotons must be decoalesced. With an initial longitudinal emittance of $3 \mathrm{eV}$-s and decoalescing into approximately 10 bunches, a $95 \%$ decoalescing efficiency has been calculated using the same methods by which coalescing performance at present is accurately predicted. No hardware are required for the decoalescing operation.

\subsection{Booster Requirements}

Because of the existence of electron cooling, the proton transverse and longitudinal emittances are not very critical for Tevatron injection. On the other hand, any transverse beam emittances much larger than approximately $20 \pi \mathrm{mmmr}$ will required a reduction in the electron cooling rate. Of dominant importance is that the Booster achieve the goal of $6 \times 10^{10}$ protons/bunch during stacking.

\subsection{Antiproton Source Requirements}

The stacking rate required for the peak and integrated luminosity goals of Tevatron* is accomplished via rapid targeting of a large collection of protons into a system which efficiently captures the resultant antiprotons. Given simultaneous fixed target operations, the time between protons on target will be approximately 2 seconds. The design goal for the number of protons per bunch on target is $6 \times 10^{10}$, which corresponds to $5 \times 10^{12}$ protons per Booster batch or per cycle. Given the required stacking rate, this yields the stacking efficiency needed to accomplish the goals of Tevatron*. Table 3.6.1 contains the parameter values anticipated for the Antiproton Source.

Table 3.6.1: Summary of Antiproton Source performance parameter values. The Maximum Accumulator Stack Size numbers in the Present and MI columns are given a 50\% additional intensity to account for the fact that the extraction method in the Accumulator prohibits the extraction of the entire stack, leaving some antiprotons left over after each injection.

\begin{tabular}{|l|c|c|c|}
\hline Parameter & Present & MI & Tev $^{*}$ \\
\hline \hline Main Injector Stacking Cycle (s) & 3 & 2 & 2 \\
\hline Proton Intensity on Target $\left(10^{10}\right)$ & 320 & 500 & 500 \\
\hline Time between Accumulator Dumps (hrs) & 12 & 12 & 0.5 \\
\hline Required Stacking Efficiency (ppm) & 14 & 13 & 18 \\
\hline Maximum Accumulator Stack Size $\left(10^{10}\right)$ & 96 & 216 & 8 \\
\hline
\end{tabular}


The antiprotons transferred to the Recycler every half hour should have transverse and longitudinal emittances consistent with the needs of the Recycler electron cooling system. Because of the needs of antiproton recycling, the electron beam radius will be large enough to encompass a $20 \pi \mathrm{mmmr}$ beam. Therefore, the beam from the Accumulator should be smaller than this value. Longitudinally, the present design criterion is a momentum spread of $10^{-3}$ or less in a slug of unbunched beam a little shorter than the circumference of the Accumulator. It is assumed that a kicker fires in the Accumulator removing the entire microstack.

\subsection{Recycler Requirements}

The Recycler must be able to accept $20 \pi \mathrm{mmmr}$ and $1 \times 10^{-3}$ fractional momentum spread antiprotons approximately every half hour. The maximum stacking intensity is $95 \times 10^{10}$ antiprotons.

At the end of the store an additional $125 \times 10^{10}$ antiprotons are injected into the Recycler. These antiprotons will have a transverse emittance of approximately $20 \pi \mathrm{mmmr}$. The momentum spread can be made small by bunch rotating in the coalescing RF just before transfer into the Recycler. The current in the ring is almost an order of magnitude smaller than the peak current in the Accumulator ring, so it is not anticipated to be a problem.

Partitioning of the antiprotons into the collider bunches is accomplished with moveable barrier bucket RF pulses. The RF voltage is quite low as long as it is assumed that the final rotation into a $53 \mathrm{MHz}$ bucket is accomplished via a bunch rotation using the coalescing RF system at $8 \mathrm{GeV}$ in the Main Injector. In essence, collider transfers of beams between the Recycler and Main Injector are $2.5 \mathrm{MHz}$ bucket-to-bucket phase locked.

Protons bunches from the Booster, 4 batches of 5 bunches each, are bunch rotated in the Booster before direct transfer into the Recycler barrier buckets. The Main Injector does not have to mediate this transfer. The bunches are then merged and cooled to eliminate any dilution, and transferred back to the Main Injector to be treated the same as the previous antiprotons. The total proton intensity would be approximately $120 \times 10^{10}$, smaller than the antiproton stack just before extraction. Again to match the electron cooling beam, the transverse emittance of the protons need to be less than $20 \pi \mathrm{mmmr}$.

\subsection{Symbol Definitions}

$\mathrm{A}_{\mathrm{a}} \quad$ Antiproton $95 \%$ invariant emittance (bunch area)

$A_{p} \quad$ Proton $95 \%$ invariant emittance (bunch area)

$A_{r} \quad$ Longitudinal $95 \%$ invariant emittance of the Recycler beam

$\alpha \quad$ Interaction point beam crossing half angle

B Number of bunches per beam

$\beta^{*} \quad$ Beta function value at the Tevatron Collider interaction points

$\beta_{\mathrm{r}} \quad$ Relativistic beam velocity

$\varepsilon_{\text {na }} \quad 95 \%$ normalized transverse antiproton emittance

$\varepsilon_{\mathrm{np}} \quad 95 \%$ normalized transverse proton emittance 
$\mathrm{f}_{\mathrm{O}} \quad$ Revolution frequency of an accelerator

$\gamma_{\mathrm{r}} \quad$ Relativistic beam energy

L Instantaneous luminosity during a store

$<$ L $>$ Average luminosity per day or week

$\mathrm{n}_{\mathrm{L}} \quad$ Number of interactions in a given bunch crossing at an interaction point

$\mathrm{N}_{\mathrm{a}} \quad$ Number of antiprotons per bunch

$\mathrm{N}_{\mathrm{p}} \quad$ Number of protons per bunch

$\mathrm{N}_{\mathrm{IR}}$ Number of interaction regions in the Tevatron Collider

$\mathrm{R}_{\text {lost }} \quad$ Rate at which particles are lost due to proton-antiproton collisions

$\mathrm{r}_{\mathrm{o}} \quad$ Classical radius of the proton $\left(=1.53 \times 10^{-18} \mathrm{~m}\right)$

$\sigma_{\mathrm{a}, \mathrm{p}}$ rms transverse beam size at the interaction point

$\sigma_{e} \quad$ rms energy spread of a beam

$\sigma_{\text {lost }}$ Cross section for all collision processes which reduce beam intensity

$t_{A p}$ Intrabeam scattering induced longitudinal emittance growth time constant

$t_{\varepsilon p}$ Intrabeam scattering induced longitudinal emittance growth time constant

$T_{c} \quad$ Time between beginning of collider stores

$\mathrm{T}_{\mathrm{f}} \quad$ Time required to dump Tevatron beam, reinject, and reestablish collisions

$\tau_{\mathrm{L}} \quad$ Luminosity Lifetime

$\tau_{\mathrm{Lp}} \quad$ Antiproton intensity lifetime due to particle collisions at the interaction points

$\tau_{\mathrm{La}}$ Proton intensity lifetime due to particle collisions at the interaction points

$\mathrm{T}_{\mathrm{O}} \quad$ Revolution period of the Recycler ring

$\mathrm{T}_{\mathrm{S}} \quad$ Duration of a Tevatron Collider store

$\xi_{\max }$ Total antiproton linear beam-beam tune shift acceptable for collider operations 


\section{Recycler Ring}

In this chapter the technical specifications of the $8 \mathrm{GeV}$ kinetic energy Recycler ring are presented. Much of the discussion is a direct result of a year long design study period in which a large number of options were considered. As a summary, table 4.0.1 contains a list of parameter values relevant to the design of the Recycler.

Table 4.0.1: Parameter list summarizing major design numbers for the Recycler ring, comparing them to the Main Injector.

\begin{tabular}{|l|c|c|c|}
\hline Parameter & $\begin{array}{c}\text { Main } \\
\text { Injector }\end{array}$ & $\begin{array}{c}\text { Recycler } \\
\text { ring }\end{array}$ & Units \\
\hline \hline Circumference & 3319.419 & 3319.419 & $\mathrm{~m}$ \\
\hline Injection Kinetic Energy & 8 & 8 & $\mathrm{GeV}$ \\
\hline Injection Momentum & 8.9 & 8.9 & $\mathrm{GeV} / \mathrm{c}$ \\
\hline Number of Protons & $3.0 \times 1013$ & $1.0 \times 1013$ & \\
\hline Number of Antiprotons & $0.8 \times 10^{12}$ & $2.5 \times 10^{12}$ & \\
\hline Maximum Beta Function & 57 & 57 & $\mathrm{~m}$ \\
\hline Maximum Dispersion Function & 1.9 & 1.9 & $\mathrm{~m}$ \\
\hline Phase Advance per Cell & 90 & 90 & $\mathrm{deg}$ \\
\hline Horizontal Tune & 26.425 & 26.425 & \\
\hline Vertical Tune & 25.415 & 25.415 & \\
\hline Natural Horz. Chromaticity & -33.6 & -33.6 & \\
\hline Natural Vert. Chromaticity & -33.9 & -33.9 & \\
\hline Transverse Admittance at Inj. & $40 \pi$ & $40 \pi$ & $\mathrm{mmmr}$ \\
\hline Longitudinal Admittance & 0.5 & 0.5 & $\mathrm{eV}-\mathrm{s}$ \\
\hline Injection Transverse Emittance & $12 \pi$ & $20 \pi$ & $\mathrm{mmmr}$ \\
\hline Injection Momentum Spread & $1 \times 10^{-3}$ & $1 \times 10^{-3}$ & \\
\hline Transition Gamma & 21.8 & 21.8 & \\
\hline Number of Straight Sections & 8 & 8 & \\
\hline Length of a Standard Cell & 34.5772 & 34.5772 & $\mathrm{~m}$ \\
\hline Length of Disp. Suppress. Cell & 25.9330 & 25.9330 & $\mathrm{~m}$ \\
\hline
\end{tabular}

\subsection{Permanent Magnet Design}

Because of the excellent work dedicated to the design of the Main Injector lattice, it has been concluded that the most efficient starting point for the Recycler lattice design is to copy the Main Injector lattice, including the magnet lengths, for the Recycler ring. In this case an $8 \mathrm{GeV}$ kinetic energy storage ring in the Main Injector tunnel would require $1 \mathrm{kG}$ dipole magnets.

The cost and complexity of standard laminated iron/copper coil magnets make that technology undesirable for a project whose goals are timely and inexpensive fabrication. After considerable study and the construction of a number of prototypes, permanent magnets became very attractive. Not only are their construction estimated to be a fraction as expensive as conventional iron/copper magnets, but the lack of cooling water, power supplies, and electrical safety systems makes ring construction much less expensive and complex, hence increasing the probability of rapid project approval and shortening the fabrication and installation schedule. 


\subsubsection{Major Design Choices}

The magnet and lattice designs present a number of interrelated design choices involving magnet field strength, choice of permanent magnet material, aperture and field quality requirements, choice of separated function or combined function lattice magnets, straight or curved magnets, and the choice of laminated or "bar stock" construction. Many of these tradeoffs were identified and discussed at a "Workshop on the Design of Permanent Magnet Synchrotrons" held at the Lawrence Berkeley Laboratory in October of 1994. A prototype magnet program has been underway at Fermilab since the successful conclusion of that workshop, and several key design options have been quantified and preliminary design choices made.

\subsubsection{Field Strength}

A Recycler ring which clones the Main Injector lattice requires two $6 \mathrm{~m}$ long $1 \mathrm{kG}$ dipoles in each half-cell. An increase in the field strength to $2 \mathrm{kG}$ halves the total length and number of dipoles. A higher field strength also reduces the sagitta of a magnet with a fixed bend angle. In practice the field strength is quasi-quantized due to the desirability of using an integral number of standard blocks of magnetic material in the design of the cross section. Small adjustments can be made in the overall length of the magnets to compensate for this.

\subsubsection{Aperture and Field Quality Requirements}

For ease of prototyping and conceptual design of the Recycler, we have chosen to duplicate the aperture and field quality specifications of the Main Injector. Thus the vertical distance between pole tips is $2.00^{\prime \prime}$, and the good-field aperture specification is

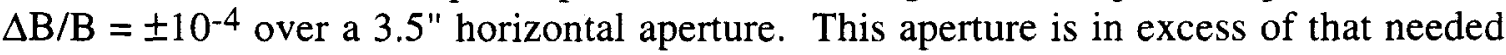
for the antiproton stacking and recycling missions of this ring. The physical aperture of the Recycler beam pipe is smaller than the Main Injector by a total of 0.25 " in width and height to allow space for a permanent $150^{\circ} \mathrm{C}$ vacuum bakeout system.

The permanent magnet design under consideration is termed a hybrid design, which means that the field is driven by blocks of permanent magnet material and the field quality is determined by accurate machining and positioning of the iron pole tips. The pole tips are effectively held at a constant value of magnetic potential (in the limit of infinite $\mu$ ), so that they are able to average out any fluctuations in the strength or placement of the permanent magnet bricks. This makes the magnet insensitive to the idiosyncrasies of individual bricks and thus easy to mass produce.

\subsubsection{Choice of Permanent Magnet Material}

The choice of magnetic material is driven by cost, required field strength, and the need for stability over time and temperature of the assembled magnet. Three materials were investigated: Samarium Cobalt, Alnico (various grades), and Strontium Ferrite. A fourth, Barium Ferrite, appeared to have no advantages over Strontium Ferrite and was not pursued. Visits were made to local factories producing Alnico and Samarium Cobalt, and discussions of magnet design optimization were held at the Lawrence Berkeley 
Laboratory workshop and with applications engineers at vendors of the various magnetic materials.

Strontium Ferrite is the preferred material and is being used for the prototype magnet program. It is more than an order of magnitude less expensive (per unit bend field) than Alnico, and two orders of magnitude less expensive than SmCo. It is available in standard grades and sizes from a variety of manufacturers. It is the material of choice for automotive applications (a modern automobile has approximately 14 lbs of ferrite for use in power windows, tachometers, windshield wipers, seat movers, and the like). This material is found widely in accelerator technology as the sputter ion pump permanent magnet bricks.

The largest standard size of ferrite brick material is a rectangle 4 "x6" $\mathrm{x} 1$ " thick, with the "easy" or magnetization axis perpendicular to the 1 " dimension. The dimensions and tolerances of these standard sizes are specified by the Magnetic Materials Producers Association. The top and bottom surfaces are ground to $\pm 0.005^{\prime \prime}$ tolerances, and the lateral dimensions are typically \pm 0.030 ". Thicknesses less than 1 " can be obtained by additional cutting and grinding of material, and thicknesses greater than 1 " must be obtained by piecing multiple blocks together. The most cost effective approach is to use an even number ( 2 or 4 ) of these 1 " thick blocks in the design of the cross section of our permanent magnets. The magnetic strength of these standard bricks is normally specified to a tolerance of $\pm 10 \%$ by the manufacturers. As discussed in section 4.1.10 measurements of brick-to-brick variation have found an rms spread within a production lot of less than $1 \%$.

The radiation resistance of Strontium Ferrite is a concern. Strontium ferrite is known not to be an activation problem from experience with its use in ion pumps. The radiation resistance of the material was claimed (by a salesman present at the Lawrence Berkeley Laboratory permanent magnet workshop) to be of order $1 \mathrm{Grad}$ for ionizing radiation and approximately $10^{7}$ neutrons $/ \mathrm{cm}^{2}$. This is the same level of radiation resistance as the epoxy used in the MI coil packs, and well in excess of that needed for an antiproton storage ring. A Cobalt-60 gamma dose of $100 \mathrm{Mrad}$ has been delivered to a brick, and has failed to induce any measurable field change in a measurement sensitive to fractional field errors of a few parts in $10^{3}$. At present the single-brick magnetic strength tester is being used to measure the radiation resistance of a sample brick before and after exposure to a realistic mix of gamma and neutron radiation in the Antiproton Source target vault.

The temperature coefficient of Strontium Ferrite was a potentially significant problem. During normal operations, the full spread in air temperature observed at any location in the Main Ring tunnel during a period of several weeks is $\pm 1^{\circ} \mathrm{C}$. During shutdowns and failures the tunnel air temperature can change by as much as $3^{\circ} \mathrm{C}$. If one allows $\Delta \mathrm{B} / \mathrm{B}=1 \times 10^{-3}$ as the worst case shift in the strength of any magnet during normal operation, this corresponds to a temperature coefficient of $\pm 0.1 \% /{ }^{\circ} \mathrm{C}$. Since temperature variations during Main Injector shutdowns or failures will occur, a coefficient of $\pm 0.05 \% /{ }^{\circ} \mathrm{C}$ is required. The $\mathrm{R} \& \mathrm{D}$ on temperature compensation of the ferrite magnets is described in a later section. As shown there, a compensation technique has demonstrated a net temperature coefficient of $-9 \mathrm{ppm} /{ }^{\circ} \mathrm{C}$, which corresponds to a $\triangle \mathrm{B} / \mathrm{B}= \pm 2 \times 10^{-5}$. 


\subsubsection{Combined Function Lattice Magnets}

One major design decision was the choice between separated function dipoles or gradient magnets. Gradient magnets in a combined function lattice eliminate up to $172 / 208=82 \%$ of the quadrupoles at the expense of additional complications in the pole tip construction, alignment, and field measurement of the magnet.

Most of the traditional arguments against combined function lattice magnets are not present for the permanent magnet Recycler. First, the magnet is operated at a fixed, low field so that iron saturation (a traditional problem with a gradient design) is unimportant. Second, the ability to be able to independently trim the quadrupole and dipole busses has been already given up. Finally, the ability to adjust the beam by physically moving any one of the gradient magnets is a possible operational advantage.

The overall attractiveness of a gradient magnet depends on the field index. The combined function design gets progressively less attractive as the gradient is increased. If the required gradient is too high, the pole tip spacing will be sufficiently uneven that a substantial amount of bend field will be lost compared to a dipole of comparable dimensions. This is a 5\% effect for the gradients needed in the arc magnets but a 15-20\% effect for the dispersion suppresser magnets which have double the ratio of gradient to bend field. The required field index also depends somewhat on the magnitude of the bend field since a high field gradient magnet will be shorter, behave more like a thin lens, and therefore require less integrated gradient to get the same phase advance per cell.

\subsubsection{Bar Stock Construction}

A major construction issue is the choice of stacked laminations or machined bar stock for the pole tips, pole tip supports, and flux return. The advantages of laminations are the well understood construction tolerances and magnetic properties, the ease of fabrication of complex shapes including magnet sagitta, as well as a great deal of local experience in design and construction. Advantages of bar stock construction are smaller assembly costs due to smaller numbers of pieces to assemble, increased mechanical rigidity, and slightly better transport of the flux with length (desirable so that the pole pieces average out block-to-block variations in the permanent magnet material). Eddy current losses which normally favor laminated construction are not present. The current prototype program is concentrating exclusively on the bar stock design, since the use of laminations is a well established practice at Fermilab with well developed performance and cost estimation mechanisms already in use.

\subsubsection{Magnet Support}

A preliminary calculation indicates that a $6 \mathrm{~m}$ long $1 \mathrm{kG}$ dipole fabricated from bar stock (see figure 4.1.1) is adequately self-supporting (sagging $0.010^{\prime \prime}-0.020 "$ ). Torsional stability is also a concern. A half cell test including magnet support brackets, beam pipe, etc. is being prepared for the MI-60 mock-up of the Main Injector tunnel.

It may be preferable to segment the magnet into sections mounted on a common girder. Each 1-2 m section would be straight, and the sagitta could be developed by the placement of the sections on the girder. The girder could provide magnetic shielding from the fringe fields of the Main Injector if this becomes important. 
The $6 \mathrm{~m}$ monolithic magnet design will be more compact than a girdered assembly. This may become a dominant consideration for integration in the tunnel. The end fields of the magnet may also be simpler to control in a monolithic design. These issues are currently undergoing engineering reviews.

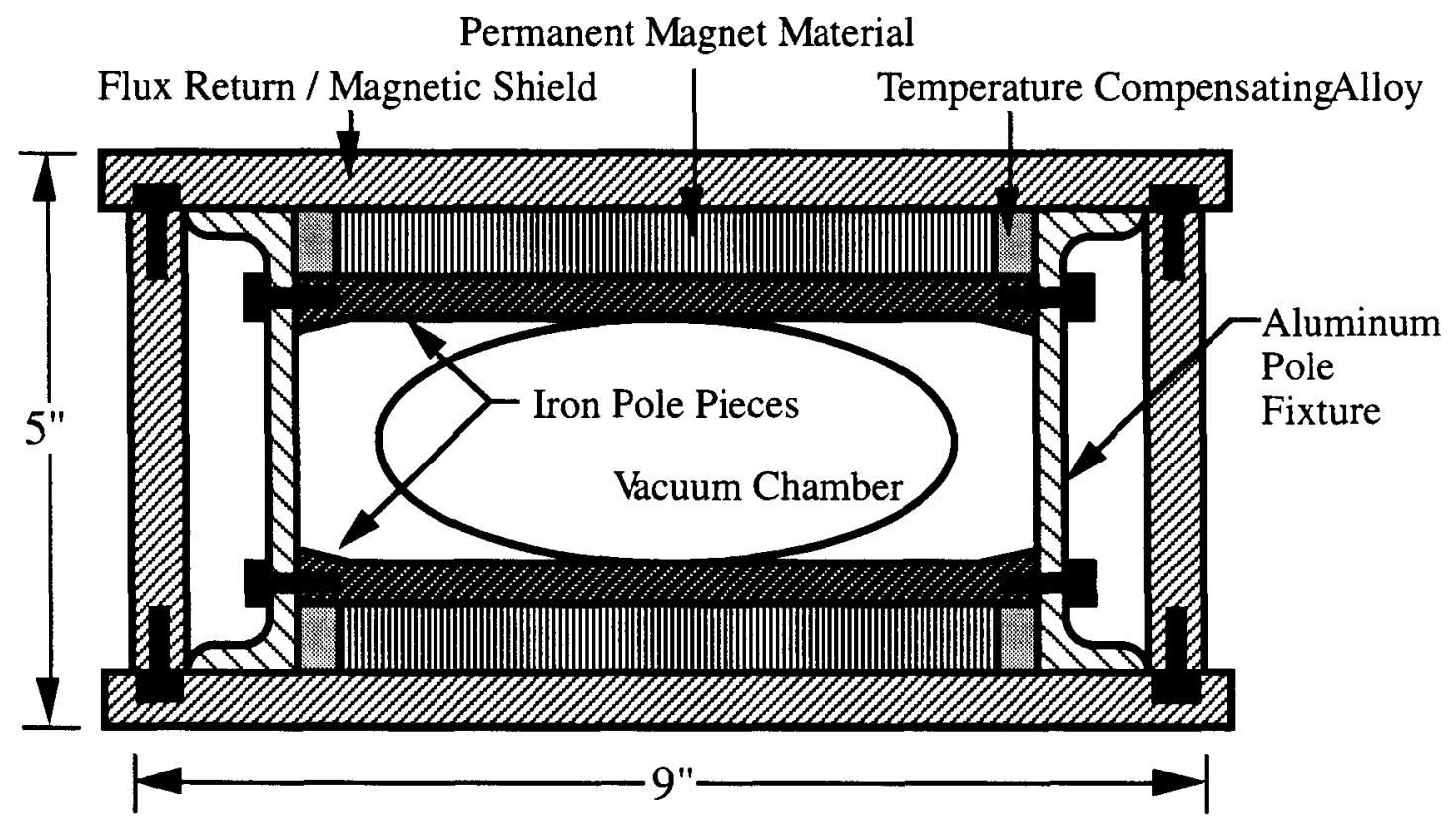

Figure 4.1.1: Cross-section of the $1 \mathrm{kG}$ hybrid permanent magnet dipole for the lattice option A shown in figure 4.1.2. This design duplicates the Main Injector beam pipe dimensions and good field aperture $( \pm 1.75$ " at $\left.\Delta \mathrm{B} / \mathrm{B}_{0}=10^{-4}\right)$. The overall dimensions are $5 " \mathrm{x} 9$ " and the weight of a $6 \mathrm{~m}$ section is approximately $2500 \mathrm{lbs}$. The field is driven by two permanent magnet blocks $5 / 8$ " thick by $6 "$ wide. The field quality is determined largely by the shape and placement of the iron pole tips located immediately above and below the beam pipe. In this design concept the pole tip spacing is set by clamping them into position against a precisely machined 2.0000" thick bar of tool steel, then bolting or pinning them to side supports made from aluminum U-channel. The flux return is fabricated from 1/2" thick bar stock and provides a "box beam" structure which provides most of the mechanical rigidity. The peak field in the flux return is approximately $6 \mathrm{kG}$.

\subsubsection{Magnet and Lattice}

Four major options have been considered for the $8 \mathrm{GeV}$ Recycler magnet and lattice design. Sketches of these options appear in figure 4.1.2.

A. Cloning the Main Injector lattice. This uses two $6 \mathrm{~m}$ long $1 \mathrm{kG}$ dipoles and one $1 \mathrm{~m}$ long $30 \mathrm{kG} / \mathrm{m}$ quadrupole in each half cell.

B. Clone the Main Injector lattice but use half the number of $2 \mathrm{kG}$ dipoles. 
C. Combined function lattice, $2 \mathrm{kG}$ bend and $5 \mathrm{kG} / \mathrm{m}$ gradient magnets. A lattice which maintains the same cell structure and bend angles, and approximately the same beta functions and dispersion as the Main Injector was developed and is described in more detail below.

D. A combined function lattice with $1.5 \mathrm{kG}$ bend and $3.5 \mathrm{kG} / \mathrm{m}$ gradient magnets, two $4 \mathrm{~m}$ magnets per half cell. This is the currently preferred option. It allows a straight beam pipe and magnet, with the magnets "split in half" around a bellows in each half cell. In addition, the $1.5 \mathrm{kG}$ field strength means the magnets are driven by a single 1" thick ferrite brick behind each pole tip, which seems optimal from the point of view of material cost, magnet weight, and effort of assembly.

The $1.5 \mathrm{kG}$ gradient magnets in option D have a width of $11.25^{\prime \prime}$, a height of $6.25^{\prime \prime}$, and a length of 13.6' (see figure 4.1.3). The sagitta (calculated from the field strength and magnet length) is $1.0 \mathrm{~cm}$, so the beam would move $\pm 5 \mathrm{~mm}$ within the vacuum chamber and magnets. The number of magnets required for the lattice in option D is 344 gradient magnets and 36 quadrupoles. The material volumes and weights in these gradient magnets are listed in table 4.1.1.

A.

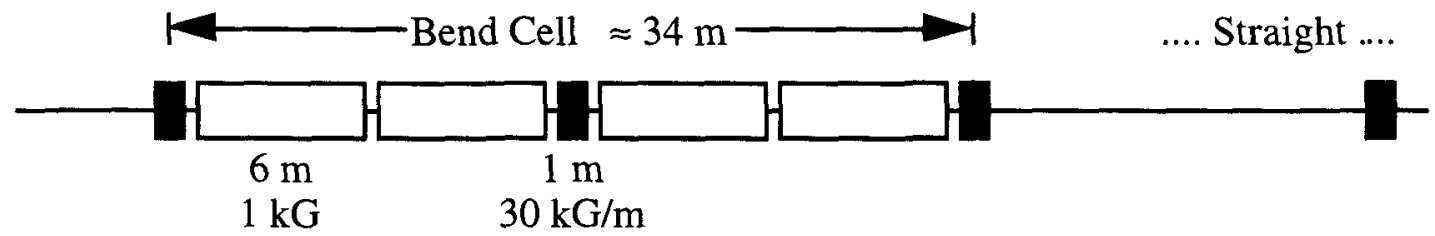

B.

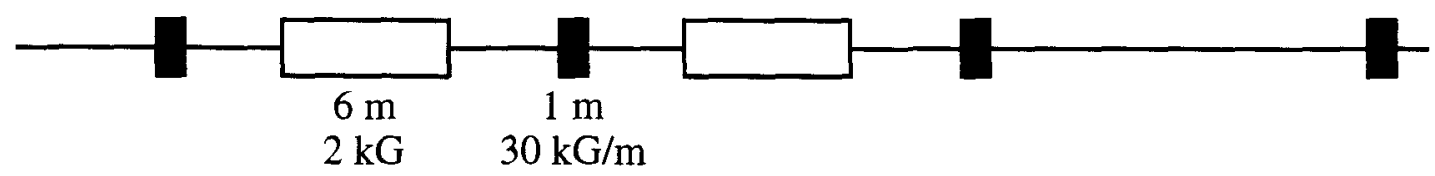

C.
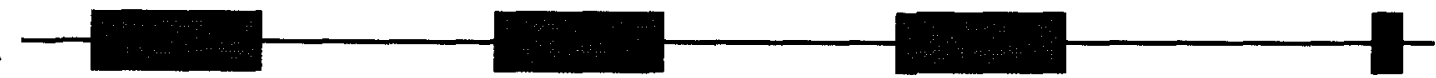

$6 \mathrm{~m}$

$2 \mathrm{kG}+5 \mathrm{kG} / \mathrm{m}$

D.

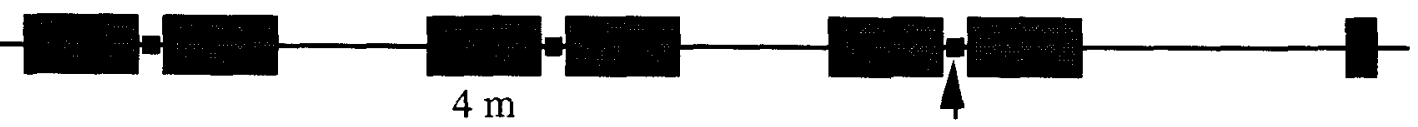

$1.5 \mathrm{kG}+3.5 \mathrm{kG} / \mathrm{m}$

Bellows

Figure 4.1.2: Sketch of the permanent magnet options which were considered in the magnet prototype program. Option A is a direct copy of the Main Injector lattice, while the combined function lattice in options $D$ is favored at this stage of the project, though the magnet strength may yet be increased toward those in option $C$. 


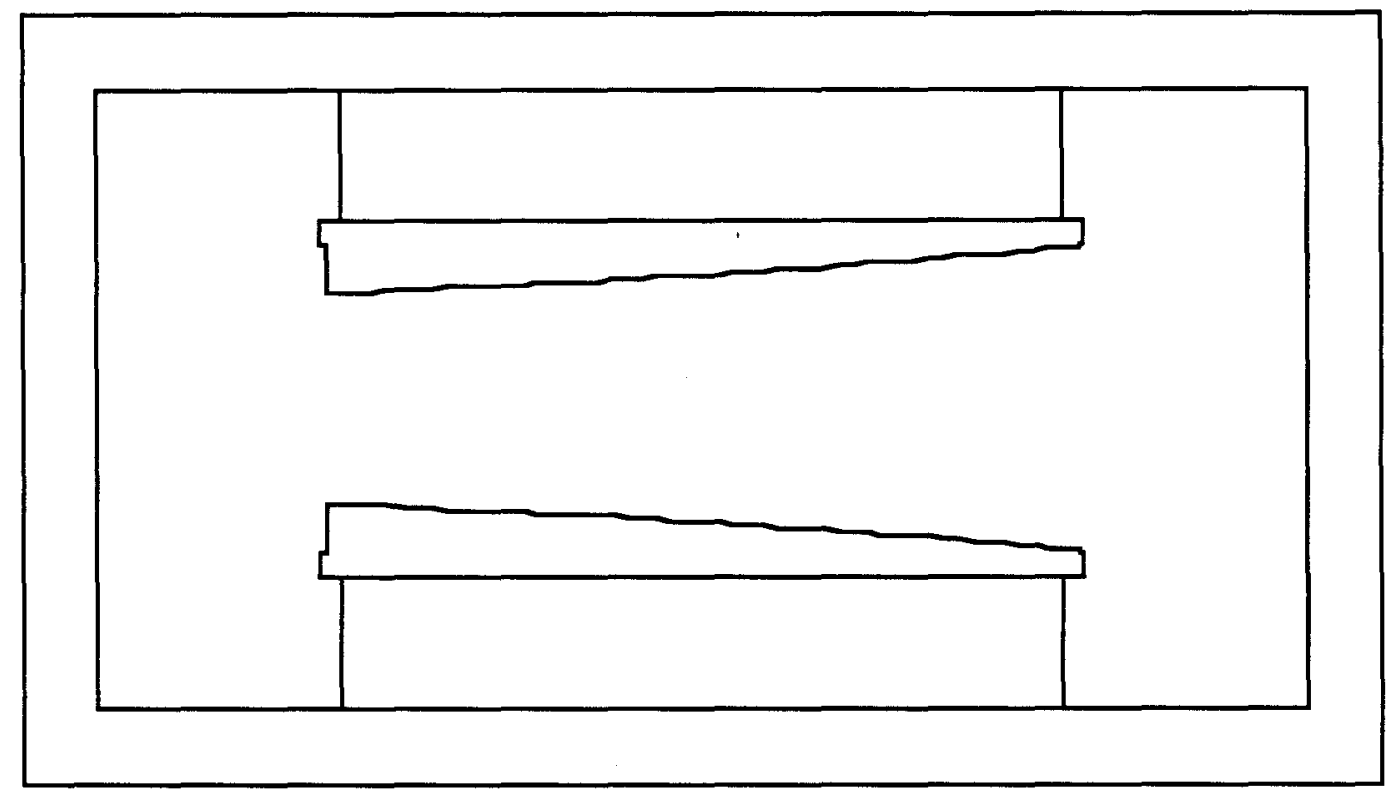

Figure 4.1.3: Cross section of the $1.5 \mathrm{kG}$ gradient magnet for option $\mathrm{D}$ outlined in the text.

The quadrupoles are required for the straight sections and as supplemental gradient in the dispersion suppression cells. The cross-section of a prototype quadrupole is shown in figure 4.1.4.

Table 4.1.1: Material cross sections and weights for the $1.5 \mathrm{kG}$ gradient magnets described in lattice design option D.

\begin{tabular}{|l|c|c|c|}
\hline Material & $\begin{array}{c}\text { CrossSection } \\
(\text { sq. in.) }\end{array}$ & $\begin{array}{c}\text { Weight } \\
(\mathrm{lb} / \mathrm{ft})\end{array}$ & $\begin{array}{c}\text { Weight } \\
(\mathrm{lb} / \mathrm{magnet})\end{array}$ \\
\hline \hline Flux Return Iron & 20.3 & 69 & 935 \\
\hline Pole Tip Iron & 7.7 & 26 & 354 \\
\hline Ferrite Bricks & 12.6 & 26 & 358 \\
\hline Aluminum Pole Supports & 5.0 & 6 & 80 \\
\hline Total & 70.3 & 127 & 1727 \\
\hline
\end{tabular}

\subsubsection{Temperature Compensation of Strontium Ferrite}

The temperature coefficient of Strontium Ferrite was a potentially significant problem. As described below, during normal operations the full spread in air temperature observed at any location in the Main Ring tunnel over a period of several weeks is approximately $\pm 1^{\circ} \mathrm{C}$. During shutdowns and failures the tunnel air temperature can drop by as much as $3^{\circ} \mathrm{C}$. If one allows $\triangle B / B=1 \times 10^{-3}$ as the worst case shift in the strength of any magnet during normal operation, this corresponds to a temperature coefficient of $\pm 0.1 \% /{ }^{\circ} \mathrm{C}$. If compensation of temperature variations during Main Injector shutdowns or failures is necessary, then a coefficient of $\pm 0.05 \% /{ }^{\circ} \mathrm{C}$ is required. The temperature 
compensation technique described below should achieve a temperature coefficient of less than $\pm 0.02 \% /{ }^{\circ} \mathrm{C}$.

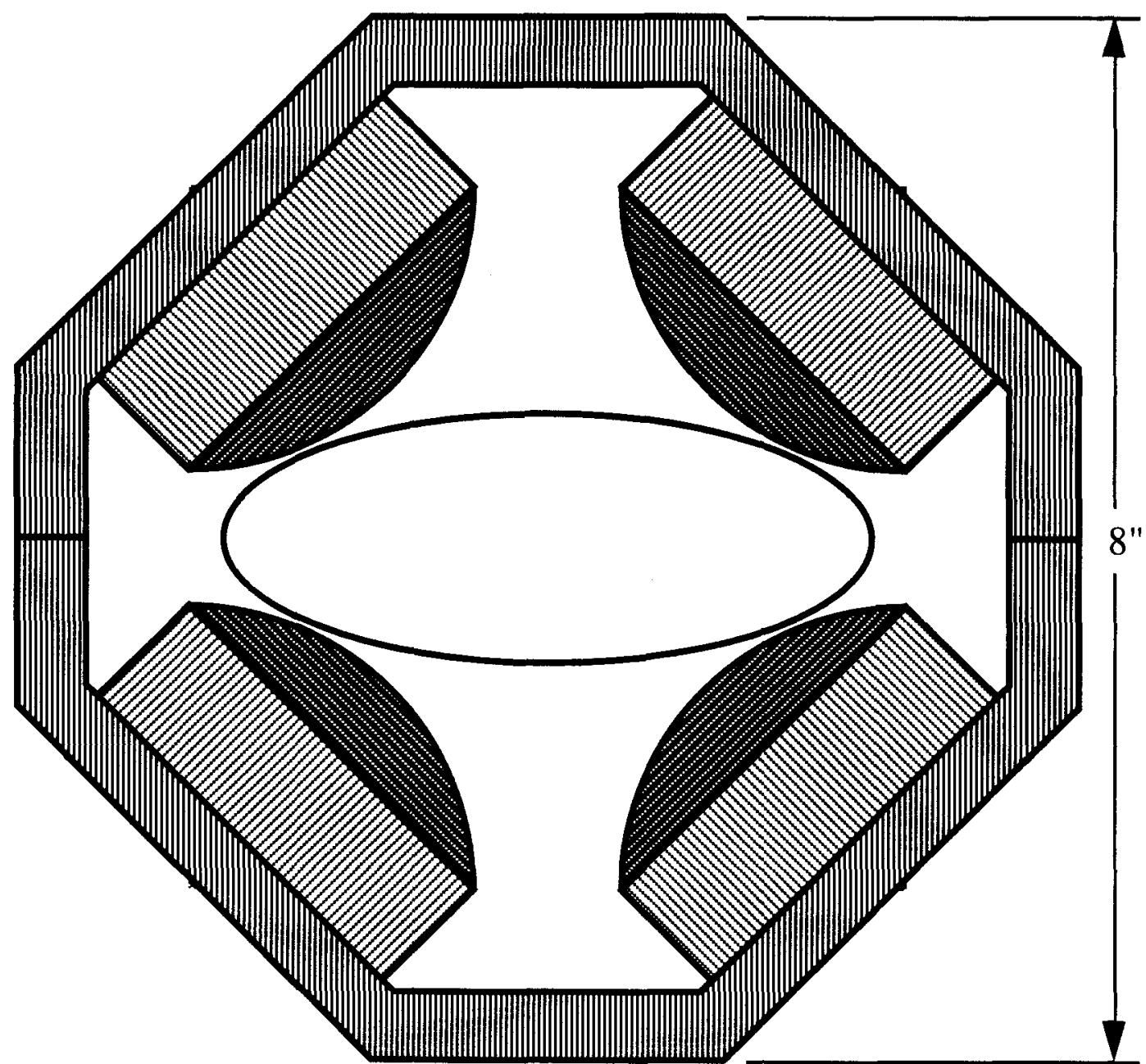

Figure 4.1.4: Cross section of a permanent magnet quadrupole. Each iron pole is powered by a brick of permanent magnet material. The inner surface of the pole tips are roughly circular with a curvature which approximates the hyperbolic profile XY=1.349 in ${ }^{2}$, used for the Main Ring/Main Injector quadrupole. The beam pipe is a 2" x 5" ellipse.

Thanks to the background datalogging capabilities of the Fermilab ACNET control system, Main Ring dipole iron, quadrupole iron, and tunnel air humidity and temperature data exists over the last year sampled every 10 seconds. Because the temperature of the permanent magnets will follow the air temperature in a very similar Main Injector tunnel, this data is quite useful for assessing whether temperature variations will be a problem in either of the storage rings being considered. 


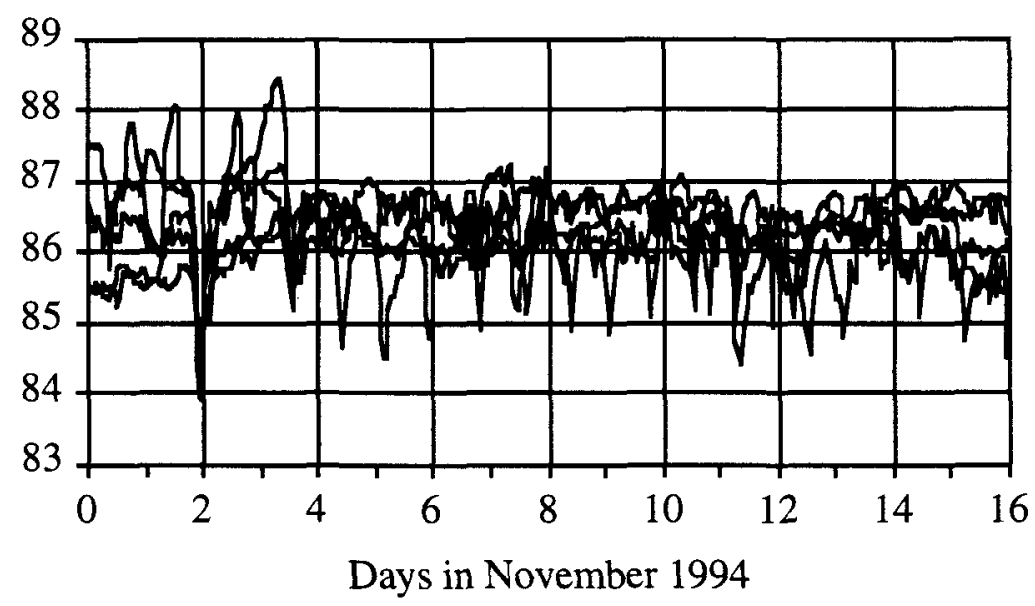

Figure 4.1.5: Air temperature at 6 monitors in the 6 sectors of the Main Ring tunnel.

Figure 4.1.5 is a plot of Main Ring tunnel air temperature data at each of the 6 machine sectors around the tunnel. Note that a given sector may change temperature by a few ${ }^{\circ} \mathrm{F}$. Given the random nature of the data in each sector, one can calculate the rms tunnel temperature variation sector-to-sector in order to model the effect of temperature variations on the closed orbit. Figure 4.1.6 contains the results of taking the standard deviation of the data from the 6 sectors. Note that in all cases the average level of the temperature in each sector was equalized to take into account any offset calibration errors. As a worst case estimate, the peak temperature variation around the ring may be of interest. The results of taking the total spread of temperature in all 6 sectors as a function of time is presented in figure 4.1.7.

\section{RMS Tunnel Variation $\left({ }^{\circ} \mathrm{F}\right)$}

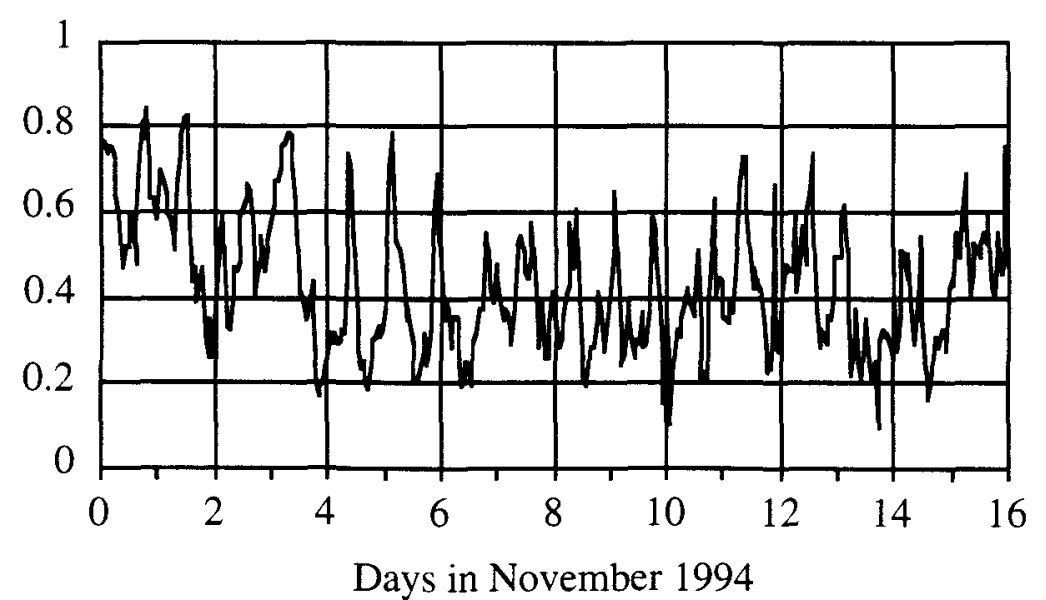

Figure 4.1.6: Standard deviation of the tunnel air temperature data shown in figure 4.1.5. 
Peak Tunnel Variation $\left({ }^{\circ} \mathrm{F}\right)$

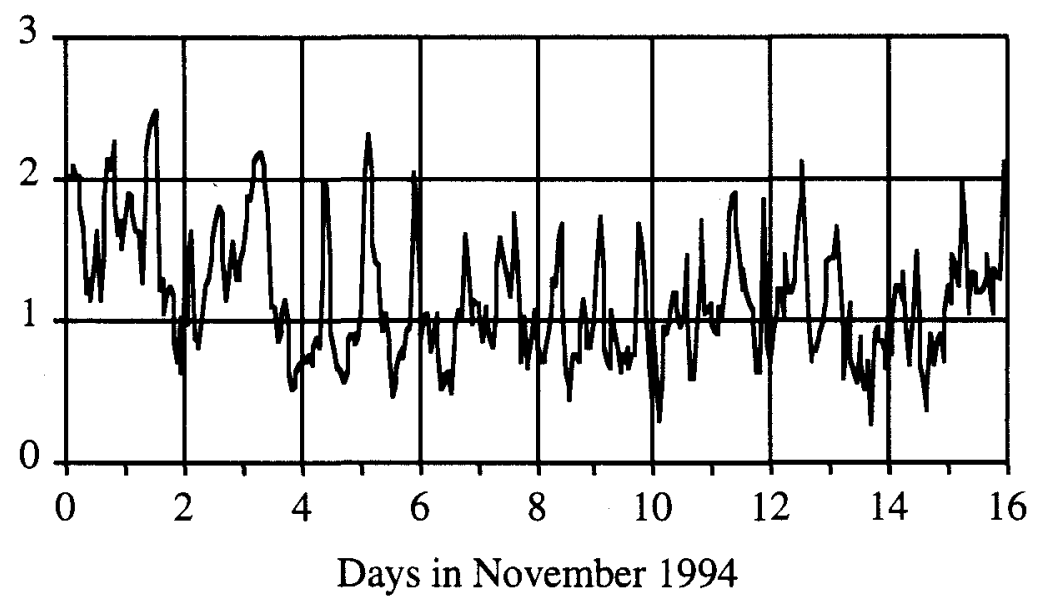

Figure 4.1.7: Peak variation of the tunnel air temperature data shown in figure 4.1.5.

Transient Response $\left({ }^{\circ} \mathrm{F}\right)$

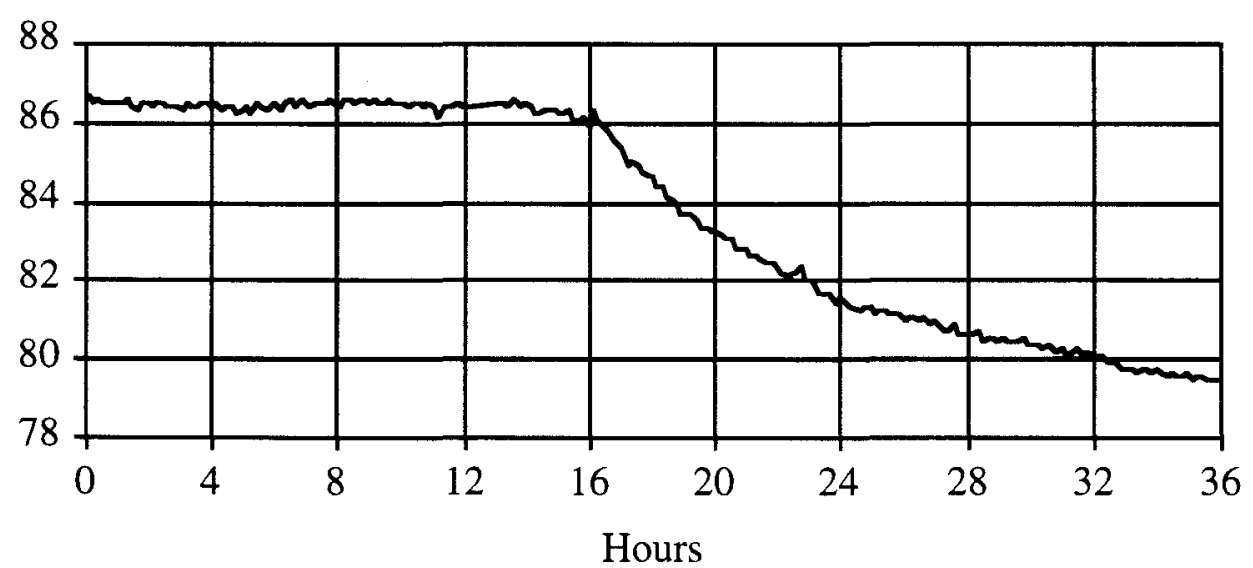

Figure 4.1.8: Transient response of the tunnel air temperature when the Main Ring ramp was switched off. The Main Ring conventional magnets are the dominant source of heat in the tunnel.

The biggest transients in the tunnel temperature $\mathrm{T}$ occur when the Main Ring dipoles are turned off. Given the model

$$
T(t)=T_{f}+\left(T_{i}-T_{f}\right) e^{-t / \tau}
$$

where $\mathrm{T}_{\mathrm{i}}=86.5^{\circ} \mathrm{F}, \mathrm{Tf}=79^{\circ} \mathrm{F}, \mathrm{t}_{\mathrm{o}}=16$ hours, and $\tau=7.5$ hours, one obtains a very good fit to the data in figure 4.1.8. The initial temperature $T_{i}$ is determined by the conduction of heat from the Main Ring magnet coils to the air and iron during nominal operations. The 
final temperature $T_{f}$ is high compared to the ground temperature, but consistent with the temperature of the LCW water transmitted around the ring for cooling purposes. The air temperature time constant of 7.5 hours is of considerable interest, since it gives one a time scale over which to stabilize the permanent magnets against large, infrequent temperature variations.

The present plan for dealing with the temperature coefficient is to include temperature dependent magnetic shunts into the design to cancel the intrinsic temperature coefficient of the ferrite material. This is a standard procedure which is applied on an industrial scale to null out the temperature coefficients of such products as automobile speedometers and power company Watt-hour meters. It allows the use of the much more cost effective ferrite permanent magnet materials in these products.

The compensation trick (pointed out by Kirk Bertsche of Fermilab) works as follows. The intrinsic temperature coefficient of the Strontium Ferrite material is $-0.19 \% /{ }^{\circ} \mathrm{C}$, i.e. the magnetic material gets weaker with increasing temperature. It turns out that Iron/Nickel alloys containing approximately $28 \% \mathrm{Ni}$ have Curie temperatures which can be controlled to be as low as room temperature. For the permanent magnet prototype effort the grade of compensator alloy which has a Curie temperature of approximately $55^{\circ} \mathrm{C}\left(130^{\circ} \mathrm{F}\right)$ was used. This material acts like air at $130^{\circ} \mathrm{F}$ and gradually turns into iron at lower temperatures. A portion of this alloy is placed in a position alongside the brick so that it short circuits part of the ferrite brick and steals flux from the pole tip in a temperature dependent manner. As the temperature rises, the ferrite material gets weaker but the shunt steals less flux since it is approaching its Curie point. In various commercial and military products this is used successfully to null out the temperature coefficient over a much wider temperature range than necessary in this application.

To demonstrate the feasibility of temperature compensation a test magnet with the correct cross section for a $2 \mathrm{kG}$ design, but only $10 \mathrm{~cm}$ long, was built. The intrinsic temperature coefficient of the permanent magnet assembly was then measured by placing the magnet in an insulated hot box, heating it, allowing the magnet to thermalize, and then gradually cooling it down. During cooldown the temperature was monitored with a platinum-wire thermometer and the magnetic field was measured with a temperature compensated Hall probe. The measured temperature coefficient of $-0.196 \% /{ }^{\circ} \mathrm{C}$ agreed well with the manufacturer's specification of $-0.19 \% /{ }^{\circ} \mathrm{C}$. Samples of the compensator alloy material were obtained from Carpenter Technology Inc., and a temperature compensation flux shunt \#1 was designed using the manufacturer's B-H vs. temperature data. It was only possible to estimate the dimensions of the shunt since the manufacturer's data did not go out the necessary values of $\mathrm{H}$. Shunt \#1 was inserted in the magnet and the cooldown test was repeated. It nulled out roughly $2 / 3$ of the temperature coefficient, reducing it to approximately $670 \mathrm{ppm} /{ }^{\circ} \mathrm{C}$ (see figure 4.1.9). A second shunt was then produced on the basis of the results from the first shunt, and it reduced the temperature coefficient to approximately $9 \mathrm{ppm} /{ }^{\circ} \mathrm{C}$.

Therefore, at present the temperature compensation technique appears to work much better than required for the Recycler ring permanent magnets. The ability to adjust one of two opposing temperature coefficients allows the nulling of temperature drifts in the magnets, including those from magnetic and mechanical effects. In two iterations it was possible to adjust the shunt on the test magnet to a net temperature coefficient of $<10 \mathrm{ppm} /{ }^{\circ} \mathrm{C}$, well below an acceptable design tolerance which is on the order of 
$200 \mathrm{ppm} /{ }^{\circ} \mathrm{C}$. The nonlinearities in the temperature coefficients of the materials did not pose a problem in canceling the temperature coefficients over the expected operating range of $25-30^{\circ} \mathrm{C}$.

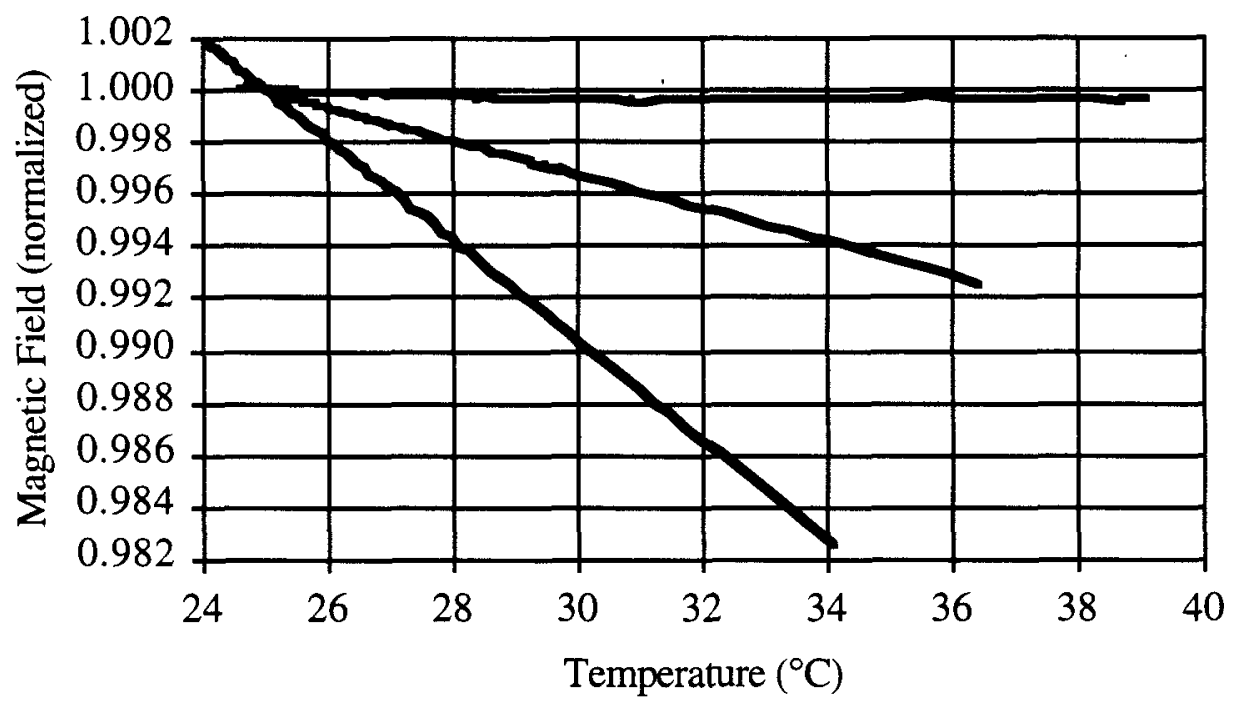

Figure 4.1.9: Data from the temperature compensation shunt test. The bottom curve is the uncompensated magnet with a temperature coefficient of $-0.2 \% /{ }^{\circ} \mathrm{C}$. The middle curve is the result of the first temperature compensating shunt, where because of a lack of material characterization the thickness of the shunt had to be extrapolated from the existing data. The top curve is the result of second shunt. The thickness for this shunt was estimated from the performance of the first shunt. Over the entire temperature range, which is much bigger than that expected in the tunnel, the average residual temperature coefficient is less than $50 \mathrm{ppm} /{ }^{\circ} \mathrm{C}$.

The total cost of the compensator alloy is roughly $300 \mathrm{k} \$$, depending on which magnet design is chosen. This is slightly more than the ferrite material itself but not prohibitive. At present four commercial sources for the compensator alloys have been identified.

The major remaining question with this technique is the extent to which the temperature coefficients of complete magnets will have to be individually trimmed in production. The manufacturers claim that the variation of the Strontium Ferrite temperature coefficient from lot-to-lot is almost nil. The amount of compensator material has to be correct within $\pm 10 \%$ to obtain the desired order of magnitude improvement in temperature coefficient. The magnet-to-magnet reproducibility of the compensation shunts will be determined by our prototype program.

\subsubsection{Unique Problems of Permanent Magnets}

The following represent potential difficulties which must be addressed in the R\&D and prototype program.

Design of field strength trimming hardware. Although the brick-to-brick variation in field strength appears to be at the $1 \%$ level (see figure 4.1.10), it will probably be necessary to develop procedures to correct the lot-to-lot variation of the permanent 
magnets. This procedure can be bypassed if the entire brick inventory is purchased at one time in a single purchase. If necessary, a simple tuning procedure can be used. It is an adjustable piece of ferromagnetic material which steals away additional flux from the pole tips. If located sufficiently far from the good field region this will adjust the field integral without affecting the field quality. The trim could also be a moveable fixture located at the magnet end. A suitably convenient dipole trim could also be used for manually correcting steering errors. An advantage of the gradient magnet design is that the field trim and steering functions are built into each magnet.

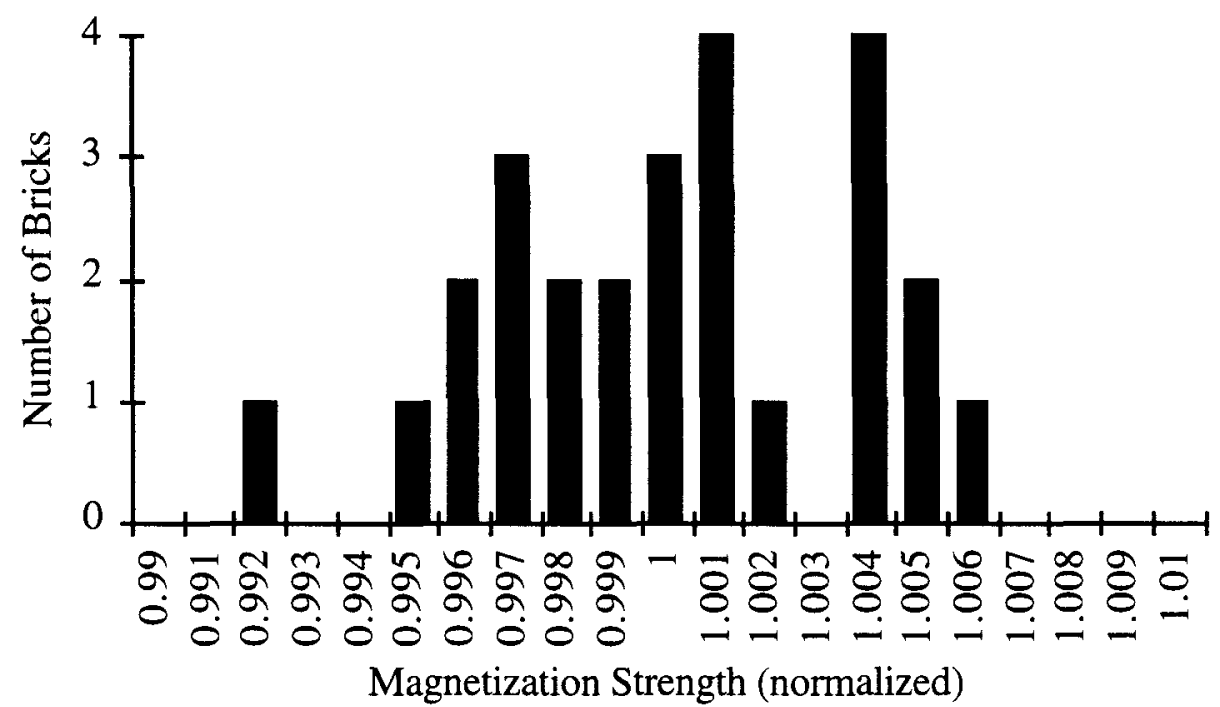

Figure 4.1.10: Histogram of the first 26 bricks tested with the MTF single brick field strength tester. The full spread of the bricks tested was $1.3 \%$ and the RMS spread was $0.3 \%$. This measurement indicates that within a single lot of bricks we can expect the variation to be less than the $\pm 10 \%$ tolerance specified by the manufacturer.

As part of the prototype program a first lot of 100 Ferrimag type 8A Strontium Ferrite blocks from Crucible Magnetics was ordered. These were magnetized at Dexter Magnetics using a remarkable crude magnetizing jig which applies $\mathrm{a} \approx 2 \mathrm{~T}$ pulsed field more or less parallel to the "easy" axis of the block. Normally ferrite blocks are shipped demagnetized from the foundry, and the distributor's shipping department will either magnetize them or not according to the customer's wishes. The ferrites can apparently be magnetized and demagnetized an indefinite number of times by the application of the $\approx 2 \mathrm{~T}$ field, with no degradation in either residual field or stability. At Fermilab's Magnet Test Facility (MTF) a single brick magnetic strength tester was developed. It drives a magnetic circuit with a load line similar to the prototype magnet design and has been used to measure the spread of brick strengths.

Residual fields in the pole tips and flux return. A field error of $1 \times 10^{-4}$ in a $1.5 \mathrm{kG}$ magnet is 0.15 Gauss. Residual magnetism in soft iron is of order 10 Gauss. For example, the flux return iron in the single brick tester has a memory of approximately 10 Gauss depending on whether the last brick was inserted "arrow up" or "arrow down". This problem is dealt with in copper driven magnets by taking the magnets through a 
hysteresis cycle which resets all magnets to a known state. No such capability exists with a permanent magnet ring. Therefore the potential exists for producing a spurious magnetization of the iron parts during magnet production or installation which corrupts the field quality and cannot be removed. This problem must be quantified and procedures for avoiding or removing spurious magnetization of the iron pieces must be developed. To date it has not been a problem to achieve a $10^{-4}$ field quality in the prototype magnets.

Qualifying vendors of permanent magnet material. At present there are several semistandard grades of permanent magnet material suitable for our uses and available on the market from alternate vendors. Each vendor's composition is proprietary, and several of our key specifications (e.g. stability over temperature and time) are not standardized. Procedures must be developed to qualify vendors. At present the vendor issue is complicated by industry-wide lead time of approximately 10 months for new large orders. The entire order for the Recycler ring represents only a few percent of U.S. annual production so it might be difficult to get the attention of large suppliers. We have obtained prototype quantities of approximately 100 bricks from four potential vendors for evaluation.

\subsubsection{Prototype Program}

The goals of the prototype program are:

1) Verify the field calculations. Status: $10^{-4}$ field shape agreement with the magnetic field calculation program POISSON. The agreement in absolute field strength is approximately $10 \%$.

2) Develop trimming schemes suitable for production. Status: Successful demonstration of controlled demagnetization to a set absolute field strength to approximately $5 \times 10^{-4}$ and gradient shims which control the quadrupole component of the gradient magnets to $\pm 1 \times 10^{-4}$. Sextupole component shims are under test.

3) Verify techniques for temperature stabilization on production sized magnets. Status: Awaiting delivery of $1000 \mathrm{lbs}$ of temperature compensation material, which is due in mid-August.

4) Gain experience in assembly to find potentially labor/cost saving design changes. Status: Assembly labor costs estimated from first three prototypes is approximately 2 man-hours/meter of magnet for "bar stock" designs.

5) Evaluate mechanical robustness of magnet: from how high can you drop it onto the floor without affecting field quality?

6) Verify that bakeout temperatures don't induce field quality errors or field strength shifts.

7) Verify mechanical sag calculations. Status: Full length flux return shells are under construction.

8) Verify calculations of magnetic shielding. 


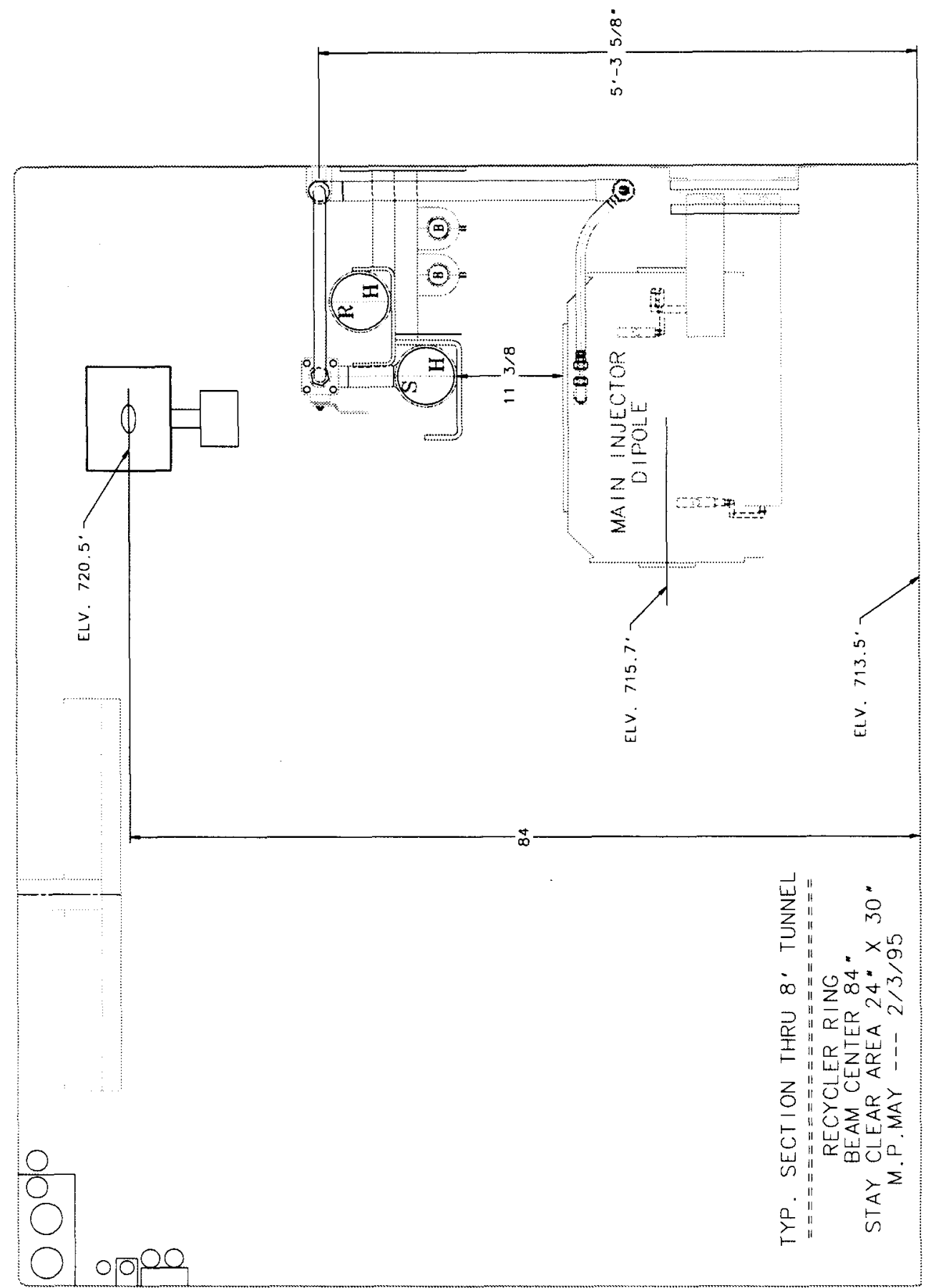

Figure 4.2.1: Mechanical drawing of a typical Main Injector tunnel crosssection. The Recycler ring has been allotted the space directly above the Main Injector magnets near the tunnel ceiling. 


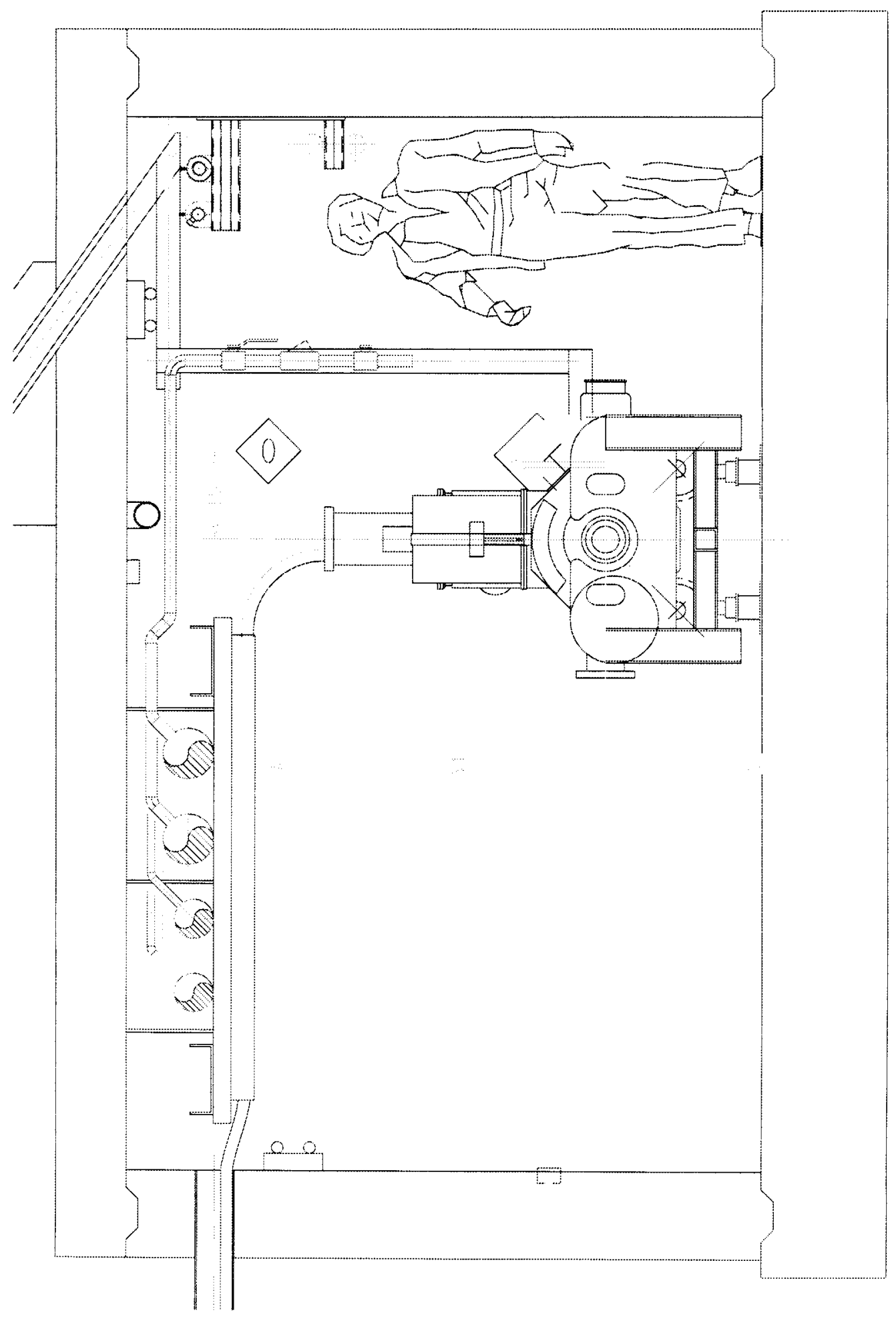

Figure 4.2.2: Mechanical drawing of the Main Injector tunnel crosssection at the MI-60 RF straight section. The Recycler ring has been moved to the radial outside of the Main Injector RF cavity power amplifiers. 
9) Develop techniques for introducing sagitta in both girdered monolithic designs. Status: The design of shorter, straight magnets has been adopted.

10) Complete end clamp design, a iron shield at the end of the magnet which ensures that the integrated magnet field is independent. of the types of equipment placed next to it.

\subsection{Tunnel Placement of Ring}

The Main Injector tunnel was designed with the capacity to hold one or two additional accelerators. For the vast majority of the circumference of the tunnel there is a region above the Main Injector magnets which is free to place another ring. The problems with ring placement occur at the RF straight and the various injection and extraction regions.

In a number of meetings with Main Injector designers and engineers, a location above the Main Injector at 7 feet above the tunnel floor has been reserved for the Recycler ring. Figure 4.2.1 shows a sketch of the tunnel in most places around the ring. The only location in the tunnel where the Recycler ring is not directly over the Main Injector is the region from MI-60 at the RF straight section to just past the proposed NUMI beamline. Figure 4.2.2 contains a sketch of the Recycler ring position at MI-60 near the RF cavities. These radial variations are adjusted so that the circumference of the Recycler ring is exactly equal to that of the Main Injector.

\subsection{Lattice}

The lattice initially considered for the Recycler ring is identical to that in the Main Injector. Two differences exist at the long straight regions at MI-60 and MI-30. At the MI-30 long straight an electron cooling channel is planned in which a horizontal and vertical beta-function as high as $200 \mathrm{~m}$ across the straight is desired. This high beta function reduces the time required to cool the injected beam and eliminates the need for quadrupoles in the middle of the straight. At MI-60 the Recycler ring must swing horizontally to the outside of the Main Injector location in order to bypass the RF power amplifiers on the Main Injector RF cavities. The ring footprint will be adjusted elsewhere to keep the circumference equal to the Main Injector value.

A combined function magnet lattice for the Recycler ring has been constructed which follows the footprint of the Main Injector and includes a high beta section in the MI-30 straight section for electron cooling. There are many options for optimizing the number, length, strength, and gradient of the gradient magnets and the lengths and gradients of the straight section quadrupoles. This lattice serves as a proof of principle. Little or no optimization of magnet parameters has been performed yet.

The current lattice replicates the standard cell length $(17.288639 \mathrm{~m})$ and dispersion cell length (12.966479 m) of the Main Injector. At each Main Injector quadrupole location in the arc, two gradient magnets are centered (with a foot spacing between them for bellows) about the Main Injector cell boundary. The spacing between the magnet pairs provides over 8 meters for position detectors, ion pumps, and other diagnostic 
equipment. The arcs use two $4 \mathrm{~m}$ magnets, the dispersion suppresser uses two $2.67 \mathrm{~m}$ magnets and the boundary between the arc and dispersion suppressers use one of each. A sextupole field component has been included in the normal cell gradient magnets for chromaticity correction. The magnets in the straight sections are $1 \mathrm{~m}$ long quadrupoles. At the interface of the straight section $0.5 \mathrm{~m}$ quadrupoles are used (with the same gradient as the straight section quadrupoles). An alternate distribution of quadrupoles could use multiple gradients and a fixed quadrupole length. The magnet requirements and parameters are summarized in table 4.3.1.

Table 4.3.1: Recycler ring magnet requirements for a combined function lattice. The value of bend field $\mathrm{B}_{\mathrm{o}}$ is $1.547 \mathrm{kG}$ in the gradient magnets. This lattice is being replaced by one in which the bend field and gradients are constant and the magnet lengths are variable.

\begin{tabular}{|l|c|c|c|c|}
\hline \multicolumn{1}{|c|}{$\begin{array}{c}\text { Magnet } \\
\text { Type }\end{array}$} & $\begin{array}{c}\text { Length } \\
(\mathrm{m})\end{array}$ & $\begin{array}{c}\text { Number } \\
\text { Required }\end{array}$ & $\begin{array}{c}\mathrm{B}_{1} \\
(\mathrm{kG} / \mathrm{m})\end{array}$ & $\begin{array}{c}\mathrm{B}_{2} \\
\left(\mathrm{kG} / \mathrm{m}^{2}\right)\end{array}$ \\
\hline \hline F gradient normal cell & 4 & 108 & 3.69 & 3.35 \\
\hline D gradient normal cell & 4 & 108 & -3.73 & -6.35 \\
\hline F gradient dispersion cell & 2.67 & 64 & 7.2 & - \\
\hline D gradient dispersion cell & 2.67 & 64 & -7.2 & - \\
\hline straight section quadrupole & 1 & 20 & 24.76 & - \\
\hline straight section half quadrupole & 0.5 & 14 & 24.76 & - \\
\hline high beta quadrupole 1 & 0.5 & 2 & 8.207 & - \\
\hline high beta quadrupole 2 & 1 & 2 & -27.96 & - \\
\hline high beta quadrupole 3 & 1 & 2 & 23.66 & - \\
\hline
\end{tabular}

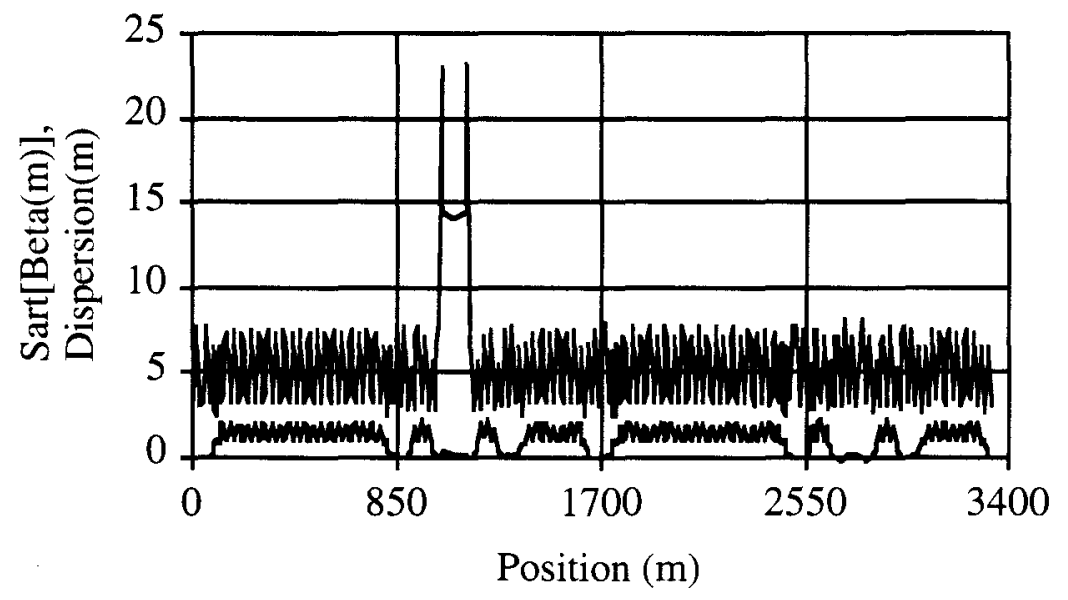

Figure 4.3.1: Vertical beta function and horizontal dispersion around the Recycler ring for the first iteration on a combined function lattice using the parameters in table 4.3.1. The horizontal beta function is similar to the vertical beta function. 


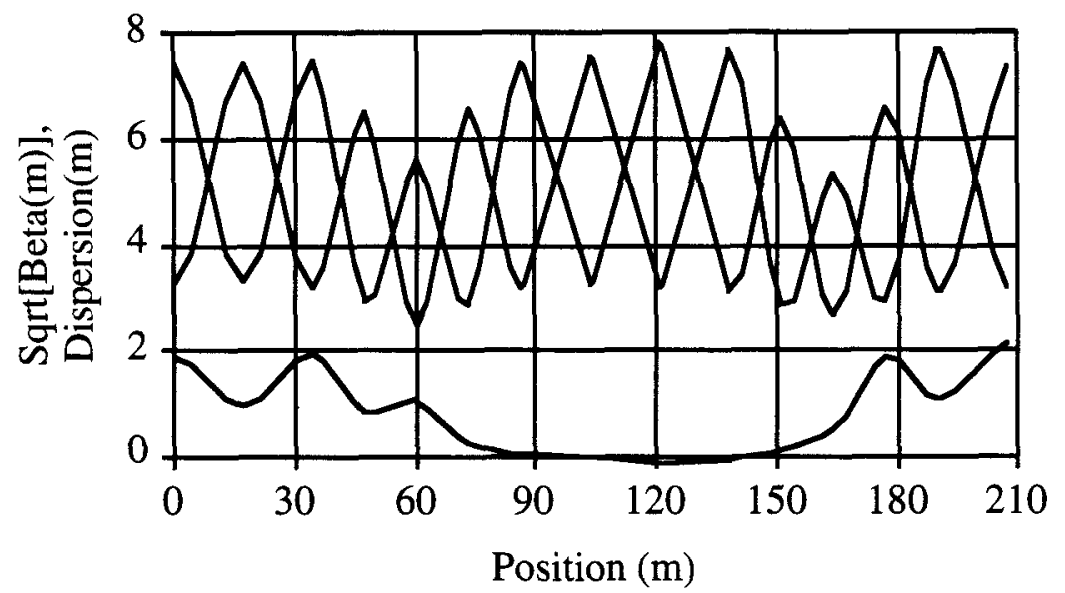

Figure 4.3.2: Beta function and horizontal dispersion in a straight section surrounded by dispersion suppression cells.

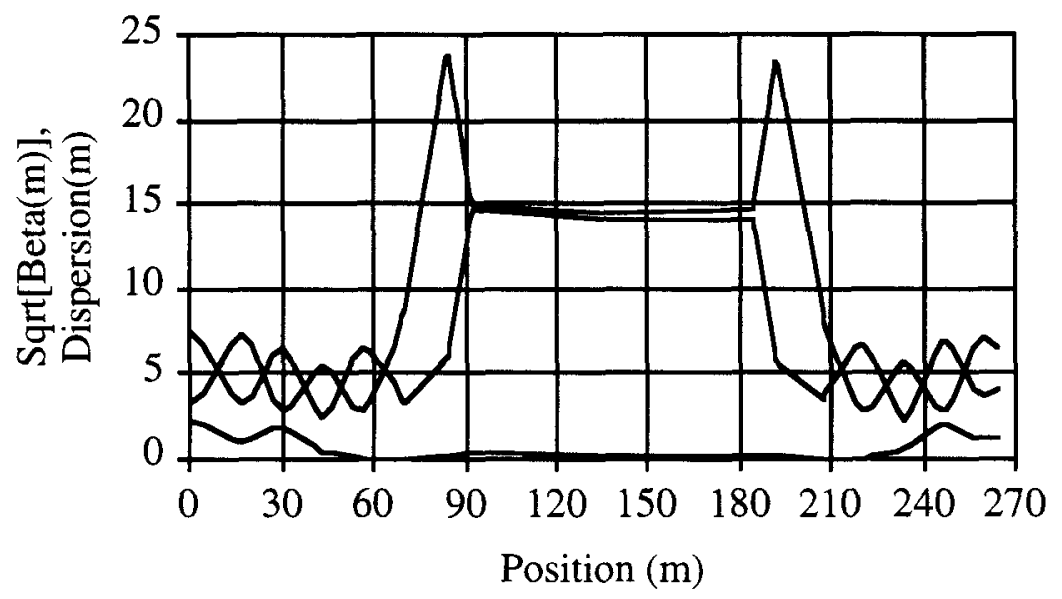

Figure 4.3.3: Lattice functions at the MI-30 straight section dedicated to electron cooling. The $200 \mathrm{~m}$ beta function in the $100 \mathrm{~m}$ long straight section is created by large aperture triplet quadrupoles on each end.

Table 4.3.2: General lattice parameters of the Recycler ring.

\begin{tabular}{|l|c|}
\hline Parameter & Value \\
\hline \hline Ring Circumference (m) & 3319.414 \\
\hline Arc Cell Maximum Beta Function (m) & 56.1 \\
\hline Arc Cell Minimum Beta Function (m) & 9.75 \\
\hline Maximum Dispersion (m) & 2.23 \\
\hline e Cooling Insert Max. Horz. Beta (m) & 210 \\
\hline e Cooling Insert Max. Vert. Beta (m) & 542 \\
\hline Beta Function in e Cooling Insert (m) & 200 \\
\hline Horizontal Tune & 25.45 \\
\hline Vertical Tune & 25.45 \\
\hline Horizontal Chromaticity & -34.7 \\
\hline Vertical Chromaticity & -36.3 \\
\hline
\end{tabular}


Figure 4.3.1 shows the lattice for the entire ring. A typical straight section, and dispersion suppression section are shown in figure 4.3.2. Figure 4.3.3 shows the high beta insert (and the dispersion suppressers on either side of the straight section) in the MI30 long straight section. The lattice parameters are summarized in table 4.3.2.

\subsection{Electron Cooling}

The two options for cooling the antiprotons in the Recycler ring are electron and stochastic cooling. In stochastic cooling, the cooling rate diminishes as the beam current increases. Since intrabeam scattering will heat the beam progressively faster as the beam current increases, the equilibrium emittance of the antiprotons will be very sensitive to the beam current. In electron cooling the rate of cooling is independent of the antiproton beam current, and so the equilibrium emittance will be smaller.

Another advantage of electron cooling is the ability to cool the transverse and longitudinal emittances of proton bunches before insertion to the Tevatron Collider. Before the significance of electron cooling was appreciated, the standard Tevatron Collider injection plan was to accelerate individual $53 \mathrm{MHz}$ bunches to $150 \mathrm{GeV}$ before coalescing them into a single collider bunch. For a given longitudinal emittance for the individual $53 \mathrm{MHz}$ bunches, the longitudinal emittance (and bunch length at $1 \mathrm{TeV}$ ) of the collider bunches can be predicted. The results of these calculations is that the bunch length during collision is too large, reducing the luminosity by approximately $40 \%$.

On the other hand, if proton coalescing occurred at $8 \mathrm{GeV}$, the coalesced bunch could be transferred to the Recycler ring, cooled longitudinally to $0.5 \mathrm{eV}-\mathrm{sec}$ over a time span of seconds to minutes, transferred back to the Main Injector, accelerated through transition to $150 \mathrm{GeV}$ where it is immediately injected into the Tevatron.

This process is not necessary for the antiprotons since the barrier bucket RF system can be used to segment slugs of charge into single bunches before transfer into the Main Injector. In effect, coalescing is a byproduct of the barrier bucket extraction method envisioned for the Recycler ring. The impact of both proton and antiproton "coalescing" in the Recycler ring on luminosity and Tevatron Collider operations has been described in Chapter 1.

\subsubsection{Introduction to Electron Cooling}

Electron cooling provides a method to reduce beam emittance simultaneously in all three degrees of freedom with an effectiveness practically independent of the intensity of the beam to be cooled. Although technically successful experiments were carried out both at CERN [1] and Fermilab [2], calculations showed that electron cooling was inferior to stochastic cooling for the large emittance, low intensity, medium energy antiproton beams produced by targeting high energy protons. The electron cooling rate is markedly higher for beams which have at the outset emittance typical of accelerator beams. Furthermore, the rate drops with the square of the collection energy, leading to the unattractive conclusion that it was necessary to decelerate the antiprotons to an energy of a few hundred $\mathrm{MeV}$ to match the cooling rate to the desired accumulation rate. \{Footnote: D. Larson observes [3] that reduction of cooling rate with increasing energy is frequently exaggerated by writing the rate formula with physical emittance as a 
parameter; see for example ref. [4]. Generally normalized emittance is the quantity of interest. The cooling time is proportional to emittance cubed. Therefore, when physical emittance is used the cooling time appears to scale as $\beta^{4} \gamma^{5}$ rather than as $\beta \gamma^{2}$ which reflects the correct scaling for comparing alternative schemes for achieving high luminosity at high energy.

However, when stochastic cooling is used to pre-cool antiprotons to suitable emittance, electron cooling becomes very attractive for a final stage of cooling and stacking an intense beam. The fact that the stochastic cooling time is proportional to the number of antiprotons whereas the electron cooling time is practically independent of it means that, despite its limitations, electron cooling is superior for obtaining very bright beams. The possibility of electron cooling as an Accumulator upgrade was foreseen at the time the TeV I design report [5] was written, but stochastic cooling achievements and electron cooling calculations in the intervening ten years have moved the appropriate location from the Accumulator to a new, larger, Recycler ring.

The electron cooling system and performance parameters are established by optimizing the division of the required cooling between stochastic pre-cooling and electron cooling. This division of labor is established by the time between transfers from the stochastic cooling rings to the electron cooler, and the emittances of the transferred antiproton beams. Although the parameters given in Table 4.4.1 are based on an optimistic extrapolation of current electron cooling technology, they stop short of the most aggressive scenario in which the electron cooling receives and stacks antiprotons every production cycle. The Accumulator is required to stack about 30 minutes. The guiding principle is to push as far as possible on the electron cooling to reduce the cost of the stochastic cooling systems. However, it remains possible to achieve the intended stacking rate with as much as a factor ten shortfall in electron beam current so long as the stochastic cooling can stack at design rate for a up to 30 minutes. Also, there are ways to apply electron cooling to a wider momentum spread without major loss of stacking rate if stochastic cooling falls short. The various possibilities between two second and 30 minute stacking cycles have not been examined in detail, but the design allows alternative routes to the desired accumulation rate. Neither cooling system is a simple duplication of existing designs. However, it is notable that $120 \mathrm{~mA}$ of $2 \mathrm{MeV}$ dc electron beam has been circulated successfully.[6] By developing the stochastic cooling to the fullest, one could still approach the $10^{12}$ per hour stacking rate with such electron beam current.

The details of the present cooling system design were strongly influenced by a workshop on electron cooling of $8 \mathrm{GeV}$ protons and antiprotons held at Fermilab 23-24 February 1995.[7] The initial concept was based on a proposal by the Indiana University Cyclotron Facility (IUCF) for cooling in the Medium Energy Booster of the SSC at $11.9 \mathrm{GeV}$.[8] One of the conclusions from the workshop was that there is very low technical risk in assuming that the cooling system will run with at least $200 \mathrm{~mA}$ after a short commissioning period. It was also concluded that the design goal was a credible and appropriate target. The obstacles identified are not limits in principle, and it is expected that experience will suggest how best to deal with them. The focus of the workshop and the present discussion is stacking of antiprotons from the production target. Other uses of the Recycler include cooling of antiprotons recovered from the Tevatron, cooling of protons for improved luminosity, and collecting high intensity polarized proton beams. The design parameters seem to be appropriate for these applications also. The 
practicability of a two-direction cooling section to accommodate either protons or antiprotons was established at the workshop.

Table 4.4.1: Parameters of Recycler electron cooling system.

\begin{tabular}{|l|c|c|}
\hline Parameter & Value & Unit \\
\hline \hline Electron Total Energy & 4.86 & $\mathrm{MeV}$ \\
\hline Antiproton Total Energy & 8.94 & $\mathrm{GeV}$ \\
\hline Relativistic Velocity & 0.994 & \\
\hline Relativistic Energy & 9.53 & \\
\hline Electron rms Invariant Emittance & 2.4 & $\pi \mathrm{mmmr}$ \\
\hline Antiproton 95\% Invariant Emittance & 9.5 & $\pi \mathrm{mmmr}$ \\
\hline Antiproton Energy Spread & \pm 3.1 & $\mathrm{MeV}$ \\
\hline Antiproton Beam Radius x & 0.01 & $\mathrm{~m}$ \\
\hline Electron Beam Radius a & 0.01 & $\mathrm{~m}$ \\
\hline Cathode Radius & 0.005 & $\mathrm{~m}$ \\
\hline Cathode Temperature & 1350 & ${ }^{\circ} \mathrm{K}$ \\
\hline Electron Current Ie & 2 & $\mathrm{~A}$ \\
\hline Electron Energy Stability & \pm 60 & $\mathrm{eV}$ \\
\hline Electron Charge Recovery Efficiency & 99.99 & $\%$ \\
\hline Length of Cooling Section & 66 & $\mathrm{~m}$ \\
\hline Ring Circumference & 3319 & $\mathrm{~m}$ \\
\hline Stopping Time $t_{\text {stop }}$ & 7.5 & $\mathrm{sec}$ \\
\hline Recycler Injection Frequency & 0.1 & $\mathrm{~Hz}$ \\
\hline Ring Vacuum & $\sim 1$ & $\mathrm{nTorr}$ \\
\hline Stray Magnetic Field & $\sim 3$ & $\mathrm{mG}$ \\
\hline
\end{tabular}

\subsubsection{Cooling Time for Electron Cooling}

When the proton velocity distribution in the beam rest frame is considerably wider than the electron velocity distribution, the cooling rate is proportional to $\left(v_{p}{ }^{*}\right)^{-2}$, where $\mathrm{v}_{\mathrm{p}}{ }^{*}$ is the beam frame (anti)proton velocity. If one integrates from initial time 0 to some cooling duration $\mathrm{t}_{\mathrm{f}}{ }^{*}$, then

$$
\mathrm{t}_{\mathrm{f}}^{*} \propto \frac{1}{\left(\mathrm{v}_{\mathrm{p}_{\mathrm{i}}}^{*}-\mathrm{v}_{\mathrm{p}_{\mathrm{f}}}^{*}\right)^{3}}
$$

Neglecting $\mathrm{v}_{\mathrm{pf}}{ }^{*}$, i. e. taking it to be practically zero, one obtains an expression for $\mathrm{t}_{\text {stop }}{ }^{*}$, the time the antiproton velocity distribution would be cooled to a point if the rate expression remained valid to that time.[3] In fact, of course, the cooling begins to slow when the distributions reach maximum overlap, and the several diffusive processes like intrabeam scattering determine a finite equilibrium velocity spread. However, for present purposes, the necessary cooling has been accomplished already by the time antiproton emittance has reached the electron beam emittance. Therefore, this time expressed in the lab frame is a good measure of the cooling time in all three degrees of freedom 


$$
t_{\text {stop }}=\frac{\gamma^{2} a^{2} \beta e \varepsilon_{\perp}^{3}}{120 \pi^{3} r_{p} r_{e} I_{e} \eta x}
$$

where $\gamma$ and $\beta$ are the Lorentz kinematic factors for both beams, $e$ is the magnitude of the electron charge, $a$ is the electron beam radius, $x$ is the antiproton beam radius, $r_{p}$ and $r_{e}$ are the classical particle radii, $\mathrm{I}_{\mathrm{e}}$ is the electron beam current, $\eta$ is the fraction of the ring circumference used for cooling, and $\varepsilon_{\perp}$ is the antiproton rms normalized transverse emittance. The formula is valid under conditions $\mathrm{a} \geq \mathrm{x}$, and transverse antiproton emittance is greater than transverse electron emittance which are satisfied for the Recycler parameters shown in Table 4.4.1. The cooling length fraction $\eta$ is based on a design for a (nearly) zero-dispersion insertion presented at the cooling workshop.[9] The cooling straight has a Courant-Snyder beta of $200 \mathrm{~m}$ and approximately $100 \mathrm{~m}$ of unobstructed drift.

\subsubsection{Electron Cooling and Stacking in the Recycler}

To stack $8 \times 10^{10}$ antiprotons injected every 30 minutes, the Recycler must at least be able to cool the large momentum error particles by $1 / 7$ of their energy offset in that time, because the Recycler has seven times the Accumulator radius. It will appear, however, that much higher longitudinal cooling will help to keep bits of the Recycler stack from being lost during injection. For the design parameters there is no problem since the cooling time $t_{s t o p}$ is approximately 20 minutes. In fact, although fast cooling is desired for the injected beam, it may be necessary to artificially heat the stack to raise the equilibrium momentum spread to a value that will be stable in the absence of cooling. This eventuality has been discussed in the IUCF SSC proposal.[8] The combination of strong cooling and controlled heating makes it possible to obtain an arbitrary momentum distribution.

Because the Recycler is larger than the Accumulator, it is attractive to simplify the stacking process by injecting into a gap in the beam. In this case there is no need to stack from an injection orbit and a standard full aperture kicker may be used. A gap is made in the beam with a so-called barrier bucket produced by a wide band RF system, the same technique now used in the Debuncher to keep the beam in a segment equal to the Accumulator circumference. The RF serves to clear the gap for a subsequent injection. The RF voltage is increased to reduce the penetration of stack particles into the gap after the most recent bunch has cleared out. The barrier bucket spans one sixth of the circumference, making a gap enough longer than the Accumulator batch to accommodate kicker rise and fall. The benefit of cooling in excess of the minimum to maintain an equilibrium momentum spread is important. When the beam is well cooled, it does not penetrate so far into the barrier. Therefore, the stacking efficiency is higher.

\subsubsection{Hardware}

The system described here is similar to that proposed by IUCF for the SSC. There are a number of differences in detail, but the approach is basically the same. The Recycler application is much more challenging in that the emittance is approximately ten times larger than proton beam emittance from the Low Energy Booster of SSC. However, there are offsetting advantages such as lower energy, longer cooling straight, and larger beta 
functions. Therefore, the electron current requirement and the problems of system design are rather similar. A Pelletron type electrostatic accelerator is used to produce a $200 \mathrm{~mA}$, $4.9 \mathrm{MeV}$, dc electron beam. Because the charging current capacity for the Pelletron is $\sim 0.2 \mathrm{~mA}$ a beam loss should not be much more than $10^{-4}$. Efficiency of this order has not yet been demonstrated for multi-ampere, few-MeV electron beams. There is development work in $\mathrm{cw}$ free electron lasers with similar beam requirements however.[11] Both the high efficiency collector and a gun capable of producing a reasonably controlled beam between $200 \mathrm{~mA}$ design current and a tune-up current of $\sim 0.2 \mathrm{~mA}$ need detailed design and development. Doing some of the developmental testing at existing facilities will allow important progress during the acquisition time for the Pelletron.

The angular spread of the electron beam is about \pm 20 microradian. The cooling will be compromised if beam-beam alignment or angular kicks delivered to the electron beam are greater than this. The space-charge driven angular growth reaches this level in about $1.5 \mathrm{~m}$. Therefore, there needs to be some gentle focusing with about this spacing. The present plan is similar to that of IUCF in using $2 \mathrm{~m}$ modules each containing focusing, beam position monitors, steering, and ion sweeping.

The 20 microradian tolerance on angular perturbations puts a stringent requirement on stray magnetic field in the cooling straight section. The magnetic rigidity of $4.87 \mathrm{MeV} / \mathrm{c}$ electrons is $\mathrm{B} \rho=162 \mathrm{Gm}$. Allowing a maximum 40 microradian bend in the $2 \mathrm{~m}$ between correctors limits the stray magnetic field to $3 \mathrm{mG}$. Therefore, the cooling section must be fully shielded with a multi-layer (probably three-layer) shield of mu-metal, except for possibly the outermost layer. The need for such good shielding discourages the use of solenoids for electron focusing in the manner of the IUCF design. Electrostatic quadrupoles are a practical alternative at $4.9 \mathrm{MeV} / \mathrm{c}$. There are other potential benefits of this approach including additional ion sweeping and the possibility of using the quadrupole electrodes also for steering. Although quad electrodes are not very good dipole plates off-axis, the steering corrections must be so good that the corrections will all be individually small and beam will never be significantly off-center.

Each $2 \mathrm{~m}$ module will constitute a single FODO cell. If one chooses a quad length of $25 \mathrm{~cm}$, the gradient needed to counteract space-charge is about $1 \mathrm{kV} / \mathrm{cm}$. The resulting optics are unlike a typical FODO channel in that the phase advance per cell is nearly zero. This is of course required to keep the beam envelope as nearly constant as possible. The concept is to just balance the single particle phase advance against the space-charge tune depression. Because the antiproton beta-functions are large, the electron beam quads must have large aperture if they are not to be the limiting aperture for the ring. If one (somewhat arbitrarily) assumes a ring acceptance of $50 \pi \mathrm{mmmr}$, the $\beta_{\mathrm{x}, \mathrm{y}}$ of $200 \mathrm{~m}$ leads to an aperture of $0.1 \mathrm{~m}$ radius. The required electrode potentials will thus be in the $10 \mathrm{kV}$ range. This level should present no difficulty in the high vacuum of the cooling Recycler. By the time one has enclosed such quads in a multi-layer magnetic shield, the cooling section will look from the outside mostly like a pipe, perhaps $40 \mathrm{~cm}$ OD.

\subsubsection{Development Program}

What is loosely referred to above as a "design" for the electron cooling system is really more of a prospectus on a program practically guaranteed to have useful results. 
The Recycler can open an entirely new era in the capabilities of the Fermilab accelerators. The development program should include at least the following components:

1. Calculations to validate parameter choice, including intrabeam and multiple scattering, beam heating, stability, ion trapping, effects of the non-linear field at e beam edge.

2. Detailed e-m design of gun and collector.

3. Design of electron optics.

The high confidence in obtaining at least $200 \mathrm{~mA}$ of electron beam supports pursuing the purchase and physical installation of the Pelletron without waiting for results from the development program. The sooner the Pelletron is available to the development effort, the more likely it is that early operation can surpass that minimum level. On the other hand, the development can proceed substantially without the immediate availability of a high voltage machine at Fermilab. There is much calculation, electrical design, and mechanical design to accomplish. There appear to be opportunities to test high voltage components at other laboratories.[11]

1. Bell et al., "Electron Cooling in ICE at CERN", NIM 190, pp 237-255 (1981)

2. Forester et al., "IEEE Trans. Nucl. Sci., NS-28, No. 3, p 2386 (1981)

3. D. Larson, "Electron Cooling at the SSC", AIP Proc. Series (1995)

4. F. Cole and F. Mills, "Increasing the Phase-Space Density of High Energy Particle Beams", Ann. Rev. Sci., 31, pp 295-325 (1981)

5. Design Report Tevatron I Project, Fermilab (1984)

6. D. Larson et al., "Operation of a Prototype Intermediate-Energy Electron Cooler", NIM, A311, pp 30-33 (1992)

7. Workshop on Electron Cooling of $8 \mathrm{GeV}$ Protons and Antiprotons at Fermilab, informal workshop, no proceedings, 23-24 February 1995, G.W. Foster and J. A. MacLachlan, organizers

8. MEBEC Group,"MEB E-Cool Design Report 1992", Indiana University Cyclotron Facility, Bloomington IN

9. D. Johnson, "High Beta Insert for MI-Like Ring", unpublished (1995)

10. J. Marriner at 23-24 February 1995 electron cooling workshop

11. Jerry Ramian of Quantum Institute (U. California at Santa Barbara) is developing a $2 \mathrm{MeV} 2 \mathrm{~A}$ dc electron beam for a free electron laser. There is a $3 \mathrm{MeV}$ Pelletron in operation at National Electrostatics Corp. Middleton WI, that might be used for some tests.

\subsubsection{Civil Construction for Electron Cooling}

The key component of the electron cooling system is a Pelletron electrostatic accelerator. The $5 \mathrm{MeV}$ Pelletron is a vertical cylindrical tank which is $22^{\prime}$ high and $10^{\prime}$ in diameter. In the Pelletron are two parallel beam pipes, one for acceleration of electrons emitted by the gun and the other for deceleration of the electrons just before recapture. The elegance of this design is that the work required for acceleration and deceleration are equal and opposite, so that the transport chains which send the charged pellets to the electrostatic domes do very little net work. The average current delivered by the chains is $1 \mathrm{~mA}$. 

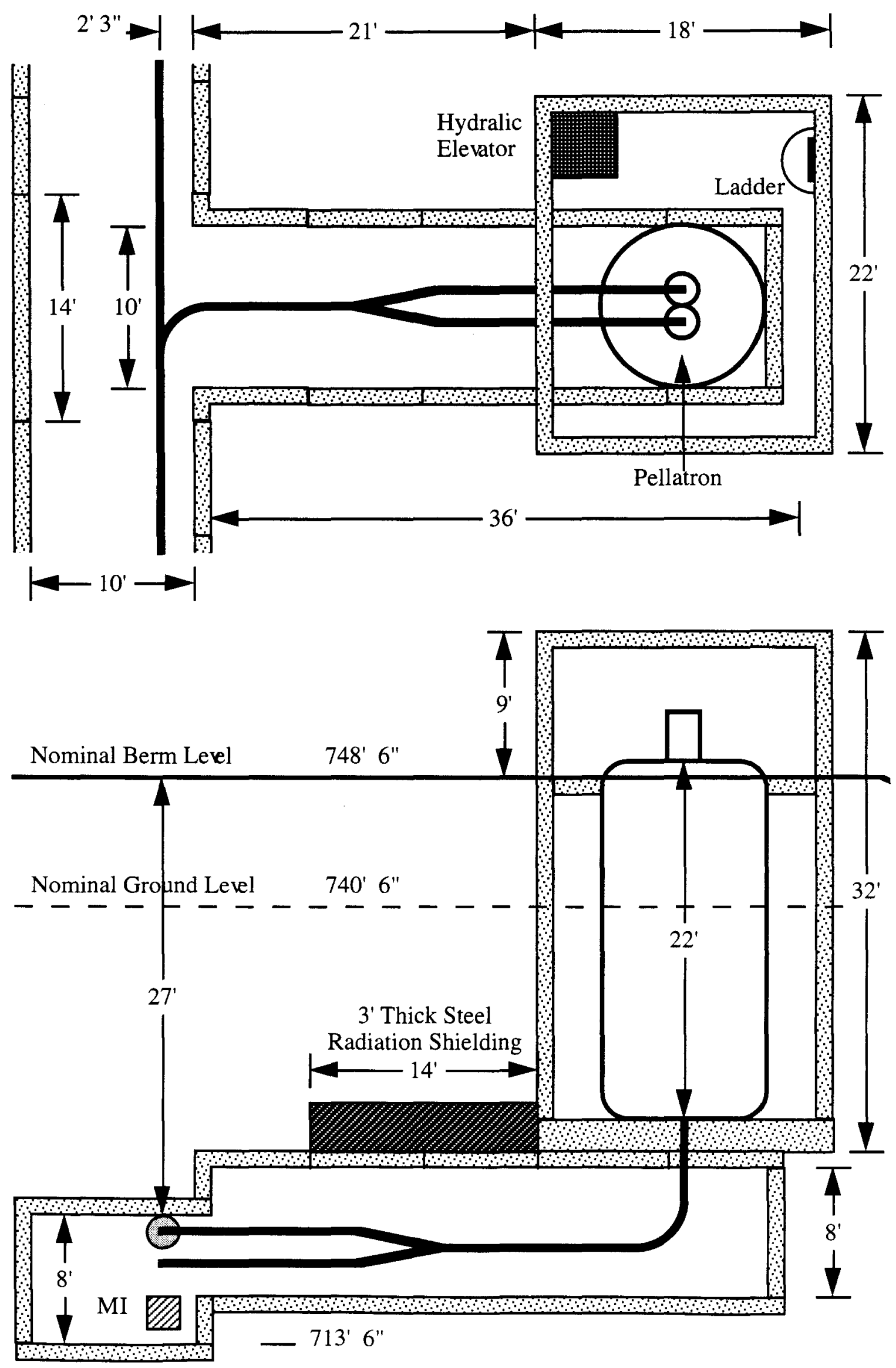
Horizontal Pelletrons also exist, but not with the dual beam pipe configuration. Instead, the acceleration and deceleration sections lie back-to-back. Therefore, the accelerator is quite large. Repairs of a horizontal Pelletron are considered awkward by the manufacturer. Finally, a horizontal Pelletron is not reversible, so electron cooling for protons would be ruled out.

The MI-30 straight section is the optimum location for the electron cooling system. Figure 4.4.1. contains a sketch of the position of the Pelletron and beam lines with respect to the Main Injector tunnel. The criterion for the placement of the Pelletrons are:

1) The maximum allowed distance to an exit in the tunnel is $50^{\prime}$.

2) The minimum shielding depth (earth equivalent) to the surface via any straight line path from the Main Injector beamline is $26^{\prime}$.

3) The minimum thickness of shield wall to the Main Injector tunnel allowing minimal occupancy in the presence of a radiation interlock detector is 14 '.

4) The Pelletron is a source of $X$-ray radiation requiring a shield wall and interlock door to exit.

5) When the Pelletron enclosure is secure, the radiation interlock detector is disabled to reduce the risk of trips of the Main Injector radiation safety system.

6) $4^{\prime}$ of steel $=12^{\prime}$ earth, $2^{\prime}$ concrete $=2^{\prime}$ earth

7) The minimum aisle space required to pass by the Pelletron is $34 "$.

8) The diameter of the shield wall penetration for the electron cooling beamlines is approximately 6 '.

\subsection{Vacuum Requirements}

In this section the vacuum system of the Recycler ring are described. Though at this time only a lumped ion pump type of vacuum system is under serious consideration, in the past a number of alternative types of system were studied. For example, a number of distributed pumping schemes were proposed and deemed either too preliminary or too risky for further discussion. On the other hand, an idea in which a titanium beam pipe is the sputtering electrode for gas ions ionized and accelerated by the beam is still being studied.

\subsubsection{Vacuum Criterion}

The criterion for the vacuum in the Accumulator storage ring is an average pressure of $1.3 \times 10^{-10}$ Torr, which translates into an $8 \mathrm{GeV}$ antiproton beam intensity lifetime of approximately 2000 hours. Because the Recycler ring is completely emptied every 4-8 hours, a considerable shorter intensity lifetime can be tolerated. Assuming a fixed injection rate of $1 \times 10^{12}$ antiprotons per hour, the circulating Recycler intensity as a function of time can be calculated for a range of intensity lifetimes. Figure 4.5.1 shows the results of a simulation of the expected Recycler stacking performance. Note that a 
beam intensity lifetime of 50 hours (average pressure of $5 \times 10^{-9}$ Torr) reduces the average stacking rate by $10 \%$, and a 20 hour lifetime (average pressure of $1.3 \times 10^{-8}$ Torr) reduces it by $20 \%$. Given this information and extrapolating from Accumulator performance, an average pressure of only $3 \times 10^{-9}$ Torr (beam lifetime of 100 hours) is desired. Just to be conservative, a vacuum pressure of $1 \times 10^{-9}$ Torr has been chosen as the design goal. An important implication of figure 4.5.1 is that if this vacuum criterion is not met initially, lets say by as much as a factor of 5 to an average pressure of $5 \times 10^{-9}$ Torr, the Recycler will still operate sufficiently well for commissioning purposes. Therefore, an additional overdesign factor for the vacuum system to compensate for installation mishaps or initial pump-down transients is not required.

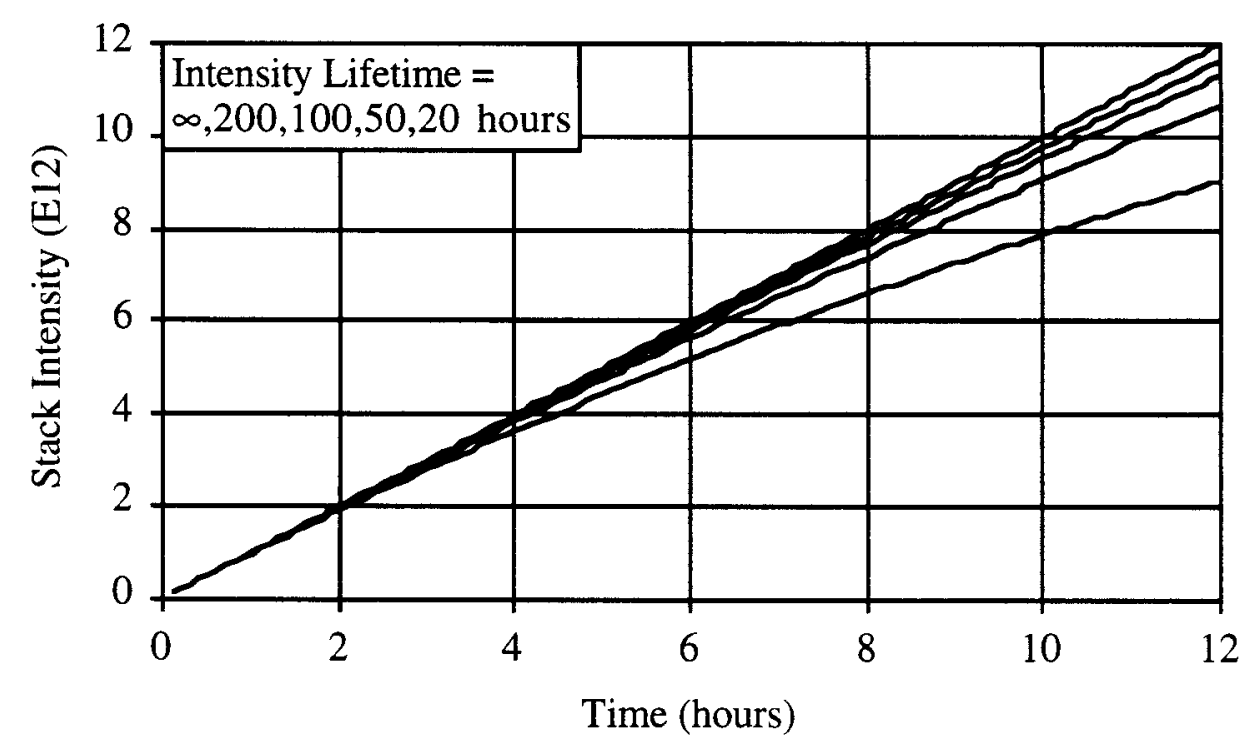

Figure 4.5.1: Results of the calculation of the Recycler intensity vs. time for a fixed injection rate of $1 \times 10^{12}$ antiprotons per hour for a number of intensity lifetimes.

\subsubsection{Vacuum Theory}

The equilibrium pressure $\mathrm{P}$ at some point $\mathrm{z}$ in the beam pipe is described by the differential equation

$$
c \frac{d^{2} P}{d z^{2}}-s P=-q
$$

where $\mathrm{c}$ is the specific conductance, $\mathrm{s}$ is the linear pumping speed, and $\mathrm{q}$ is the specific outgassing rate. The general solution of equation (1) is

$$
P(z)=A \exp \left[\sqrt{\frac{s}{c}} z\right]+B \exp \left[-\sqrt{\frac{s}{c}} z\right]+\frac{q}{s}
$$


In these calculations the pressure $\mathrm{P}$ has the units of Torr. The MKS unit of pressure which is also widely used is the Pascal $(\mathrm{Pa})$ which is equal to $\mathrm{N} / \mathrm{m}^{2}$. Other units of vacuum are bars and atmospheres. For reference the relationships between these units are displayed in figure 4.5.2. In this paper the pressures which are of interest range from $10^{-}$ 9 Torr and below.

\begin{tabular}{cc}
\hline $1 \mathrm{mbar}=100 \mathrm{~Pa}$ & 1 Torr $=133.33 \mathrm{~Pa}$ \\
$1 \mathrm{~atm}=1.013 \times 10^{5} \mathrm{~Pa}$ & 1 Torr $=1.3 \times 10^{-3} \mathrm{~atm}$
\end{tabular}

Figure 4.5.2: Relationships between the various units of pressure.

The rate of gas flow $\mathrm{Q}$ past a specific location $\mathrm{z}$ in the vacuum system is determined by the resistance to gas propagation induced by the vacuum chamber walls. The conductance $\mathrm{C}$ of a section of chamber is defined as

$$
\mathrm{C}=-\frac{\mathrm{Q}}{\Delta \mathrm{P}}
$$

When sections $n=1,2, \ldots, N$ of beam pipe are placed in series as shown in figure 4.5.3, the total conductance of the entire length is equal to

$$
\frac{1}{C}=\sum_{n=1}^{N} \frac{1}{C_{n}}
$$

As a length of a given section of beam pipe is increased, its conductance $\mathrm{C}$ decreases inverse proportionally. It is therefore useful to introduce the concept of specific conductance $\mathrm{c}$, which is related to the conductance $\mathrm{C}$ and the section length $\mathrm{L}$ by

$$
\mathrm{C}=\frac{\mathrm{c}}{\mathrm{L}}
$$

The relationship between gas flow rate, pressure, and specific conductance is derived from equation (4.5.3) to be

$$
\mathrm{Q}(\mathrm{z})=-\mathrm{c} \frac{\mathrm{dP}}{\mathrm{dz}}
$$

The equation which describes the specific conductance of a round vacuum chamber of radius $R$ is

$$
\mathrm{c}[\mathrm{m}-1 / \mathrm{s}]=0.928(\mathrm{R}[\mathrm{cm}])^{3} \sqrt{\left(\frac{\mathrm{T}\left[{ }^{\circ} \mathrm{K}\right]}{300}\right)\left(\frac{28}{\mathrm{M}}\right)}
$$


while the equation for an elliptical beam pipe of half height $a$ and half width $b$ is

$$
c[\mathrm{~m}-1 / \mathrm{s}]=1.30 \frac{(\mathrm{a}[\mathrm{cm}] \mathrm{b}[\mathrm{cm}])^{2}}{\sqrt{\mathrm{a}^{2}+\mathrm{b}^{2}}} \sqrt{\left(\frac{\mathrm{T}\left[{ }^{\circ} \mathrm{K}\right]}{300}\right)\left(\frac{28}{\mathrm{M}}\right)}
$$

The value of the molecular mass $\mathrm{M}$ for $\mathrm{N}_{2}$ is 28 . For example, a $5 \mathrm{~cm}$ radius round vacuum chamber has a nitrogen specific conductance at room temperature of 116 meterliter/sec.

The surface outgassing rate depends on the surface cleanliness and temperature treatment of the chamber wall. The surface outgassing rate $\sigma_{\mathrm{q}}$ for a clean metallic surface such as aluminum, copper, or aluminum is approximately $1 \times 10^{-12} \mathrm{~T}-1 /\left(\mathrm{s}-\mathrm{cm}^{2}\right)$. The outgassing rate for a non-baked but ultrasonically cleaned stainless steel pipe has been measured to be 5 times that minimum value. Therefore, in situ baking is an absolute necessity. To convert from the surface outgassing rate $\sigma_{\mathrm{q}}$ to the specific outgassing rate $\mathrm{q}$ it is necessary to multiply $\sigma_{\mathrm{q}}$ by the surface area per unit length. For an elliptical beam pipe

$$
\mathrm{q}[\mathrm{T}-1 / \mathrm{s}-\mathrm{m}]=\left(100 \pi \sqrt{2\left(\mathrm{a}[\mathrm{cm}]^{2}+\mathrm{b}[\mathrm{cm}]^{2}\right)}\right) \sigma_{\mathrm{q}}\left[\mathrm{T}-1 / \mathrm{s}-\mathrm{cm}^{2}\right] .
$$

The linear pumping speed $s$ has units of liter/sec-m. For a relatively short pump section such as a lumped ion pump, multiplying by the length of the pump yields the pumping speed $S$ of the pump in liter/sec.

One can define the state vector $(\mathrm{P}, \mathrm{Q}, 1)$ at any point $\mathrm{z}$ in the vacuum system. Because the constants $\mathrm{A}$ and $\mathrm{B}$ in equation (4.5.2) are piece-wise constant, that solution can be rewritten in the form of the transfer matrix

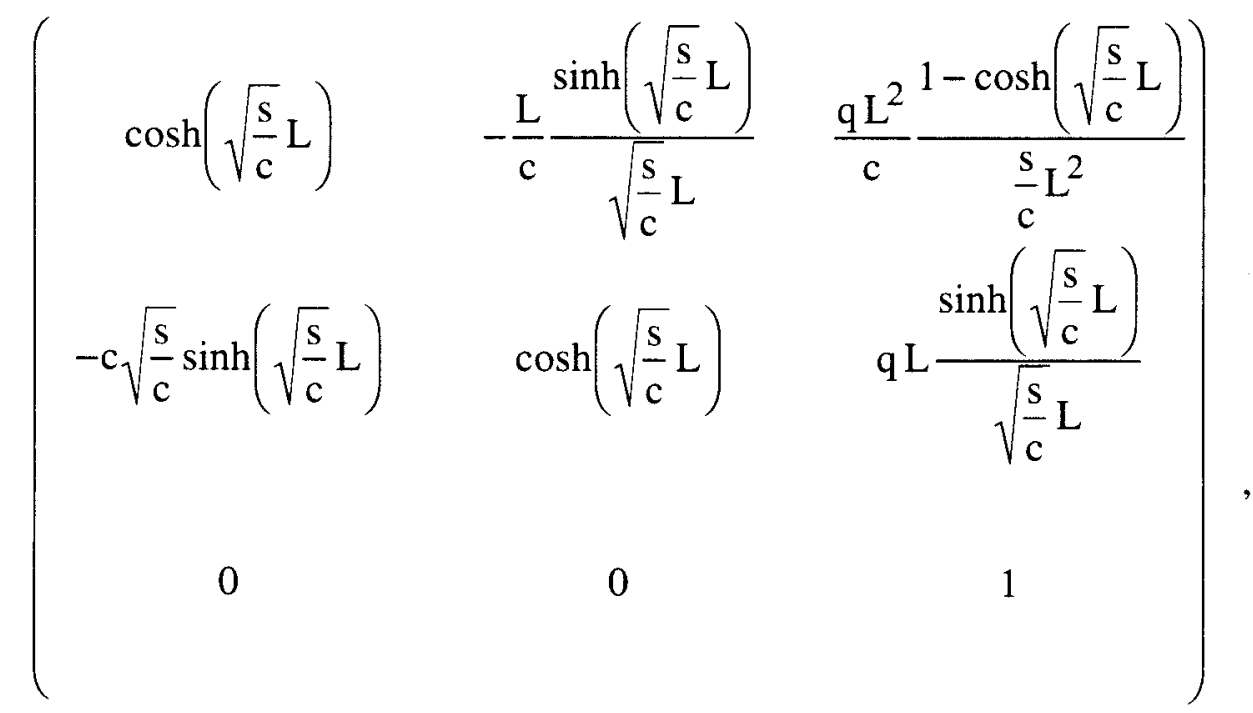

which propagates the state vector across any beam pipe section of length $\mathrm{L}$. In a section without pumping $\mathrm{s} \rightarrow 0$. Plugging this limit into the general transfer matrix in equation (4.5.10) yields the result 


$$
\left(\begin{array}{ccc}
1 & -\frac{\mathrm{L}}{\mathrm{c}} & -\frac{\mathrm{qL} \mathrm{L}^{2}}{2 \mathrm{c}} \\
0 & 1 & \mathrm{qL} \\
0 & 0 & 1
\end{array}\right)
$$

Given the top matrix rows in equations (4.5.10) and (4.5.11) the average pressure in a length of beampipe can be calculated by performing the integral

$$
\langle P\rangle=\frac{1}{L} \int_{0}^{L} P(z) d z=\frac{1}{L} \int_{0}^{L}\left[R_{11}(z) P_{o}+R_{12}(z) Q_{o}+R_{13}(z)\right] d z,
$$

where $R_{11}, R_{12}$, and $R_{13}$ refer to the matrix element values in the top row and $P_{0}$ and $Q_{0}$ are the pressure and gas flow rate at the beginning of the vacuum section.

\subsubsection{Lumped Ion Pump System}

Given the criterion of an average vacuum less than $1 \times 10^{-9}$ Torr, the spacing of lumped ion pumps is determined for an assumption of the value of the specific outgassing rate. Figure 4.5.3 contains sketches of some possible ion pump spacing options. The expected vacuum performance of these options are listed in table 4.5.1. The horizontal size of the beam pipe is partially determined by vacuum considerations. Note that the good field region is smaller than the Main Injector beam pipe size. Table 4.5.2 shows calculation results which show that the smaller the pipe, the less the outgassing area and the better the average vacuum. The conductance of the pipe is more than enough even when the pipe is as narrow as the required good field width.

Table 4.5.1: Results of vacuum calculations assuming a chemically cleaned and baked beam pipe whose width is equal to the Main Injector good field width criterion adopted by the Recycler ring.

\begin{tabular}{|l|c|c|c|c|}
\hline Parameter & $\begin{array}{c}1 \text { Pump/ } \\
\text { Half Cell }\end{array}$ & $\begin{array}{c}2 \text { Pumps } \\
\text { Half Cell }\end{array}$ & $\begin{array}{c}\text { 3 Pumps } \\
\text { Half Cell }\end{array}$ & $\begin{array}{c}2 \text { Pumps/ } \\
\text { Bellow }\end{array}$ \\
\hline \hline Lumped pump pumping speed (1/s) & 30 & 30 & 30 & 60 \\
\hline Specific outgassing rate (T-1/s-cm 2 & $1 \times 10^{-12}$ & $1 \times 10^{-12}$ & $1 \times 10^{-12}$ & $1 \times 10^{-12}$ \\
\hline Distance between pumps (m) & 17.3 & 8.65 & 5.77 & 17.3 \\
\hline Total width of elliptical pipe (in.) & 3.75 & 3.75 & 3.75 & 3.75 \\
\hline Total height of elliptical pipe (in.) & 1.75 & 1.75 & 1.75 & 1.75 \\
\hline Specific conductance (m-1/s) & 27.71 & 27.71 & 27.71 & 27.71 \\
\hline Pressure at lumped pumps (T) & $1.3 \times 10^{-9}$ & $6.3 \times 10^{-10}$ & $4.2 \times 10^{-10}$ & $6.3 \times 10^{-10}$ \\
\hline Pressure midway between pumps & $4.2 \times 10^{-9}$ & $1.4 \times 10^{-9}$ & $7.5 \times 10^{-10}$ & $3.6 \times 10^{-9}$ \\
\hline Average pressure (T) & $3.2 \times 10^{-9}$ & $1.1 \times 10^{-9}$ & $6.4 \times 10^{-10}$ & $2.6 \times 10^{-9}$ \\
\hline
\end{tabular}


Table 4.5.2: Results of vacuum calculations assuming a cleaned and baked beam pipe. The width is varied for a fixed distance between pumps with a fixed pumping speed.

\begin{tabular}{|l|c|c|c|c|}
\hline Parameter & $2^{\prime \prime} \times 3^{\prime \prime}$ & $2^{\prime \prime} \times 4^{\prime \prime}$ & $2^{\prime \prime} \times 5^{\prime \prime}$ & $2^{\prime \prime} \times 6^{\prime \prime}$ \\
\hline \hline Lumped pump pumping speed (1/s) & 30 & 30 & 30 & 30 \\
\hline Specific outgassing rate (T-l/s-cm $\left.{ }^{2}\right)$ & $1 \times 10^{-12}$ & $1 \times 10^{-12}$ & $1 \times 10^{-12}$ & $1 \times 10^{-12}$ \\
\hline Distance between pumps (m) & 8.65 & 8.65 & 8.65 & 8.65 \\
\hline Total width of elliptical pipe (in.) & 3 & 4 & 5 & 6 \\
\hline Total height of elliptical pipe (in.) & 2 & 2 & 2 & 2 \\
\hline Specific conductance (m-l/s) & 26.59 & 38.11 & 49.45 & 60.63 \\
\hline Pressure at lumped pumps (T) & $5.8 \times 10^{-10}$ & $6.9 \times 10^{-10}$ & $8.1 \times 10^{-10}$ & $9.2 \times 10^{-10}$ \\
\hline Pressure midway between pumps & $1.3 \times 10^{-9}$ & $1.3 \times 10^{-9}$ & $1.3 \times 10^{-9}$ & $1.4 \times 10^{-9}$ \\
\hline Average pressure (T) & $1.0 \times 10^{-9}$ & $1.1 \times 10^{-9}$ & $1.2 \times 10^{-9}$ & $1.3 \times 10^{-9}$ \\
\hline
\end{tabular}

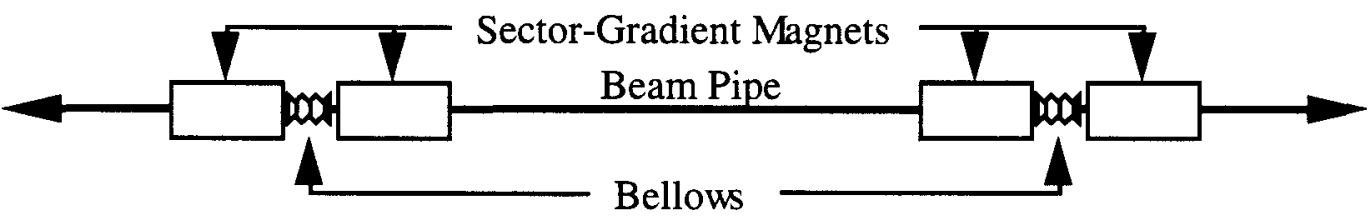

1 Lumped Pump per Half Cell

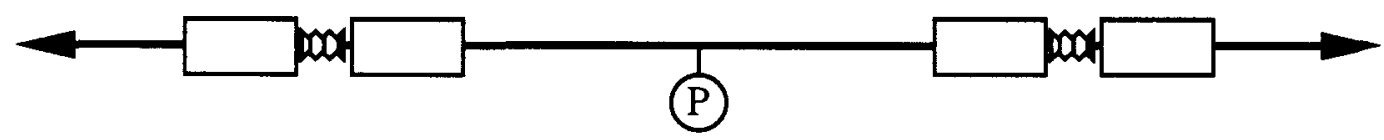

\section{Lumped Pumps per Half Cell}

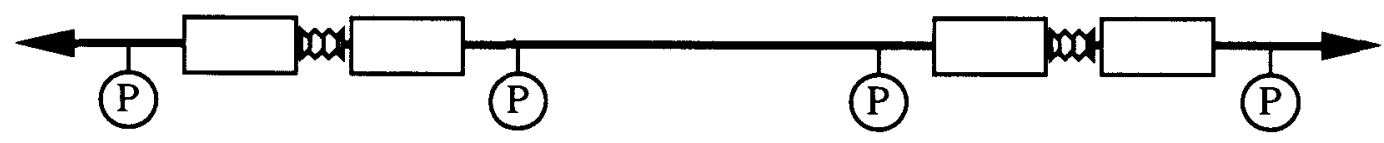

3 Lumped Pumps per Half Cell

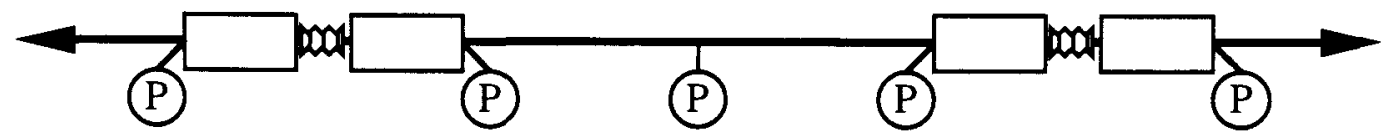

2 Lumped Pumps per Half Cell at Bellows

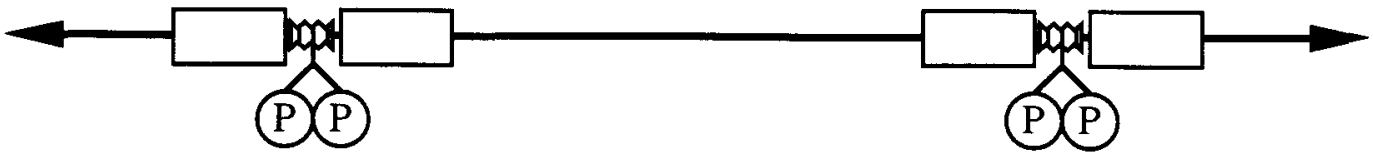

Figure 4.5.3: Various pump spacing options envisioned for the Recycler 


\subsubsection{Vacuum Test}

A test is underway to determine the ability to attain a surface outgassing rate of $1 \times 10^{-12}$ Torr-liter/(sec-cm $\left.{ }^{2}\right)$. The steps involved in the test are:

1) Clean the beam pipe. This was accomplished at Argonne for the purposes of this test. The system was then pumped down and the vacuum profile measured. The vacuum profile is plugged into equation (4.5.10) to calculate the specific outgassing rate.

2) Bake to $150^{\circ} \mathrm{C}$ and again measure the vacuum profile. This simulates the results expected in the tunnel just after installation of the ring.

3) Bake to $400^{\circ} \mathrm{C}$ and again measure the vacuum profile. This would not be done in the tunnel, but would indicate the necessity of a hydrogen bake before beam pipe installation.

4) Let the system up to air, bake it at $150^{\circ} \mathrm{C}$ and measure the vacuum profile. This step would predict the Recycler vacuum system performance with hydrogen degassed pipe after an in situ bake.

\subsubsection{In-situ Baking}

Thermal calculations have been performed to assess the ability to bake the beam pipe to $150^{\circ} \mathrm{C}$ in situ without the permanent magnetic material heating up to more than $50^{\circ} \mathrm{C}$ or so. Preliminary data suggests that $1 \mathrm{~W} / \mathrm{cm}$ is sufficient to heat the pipe, and the magnetic material never exceeds a temperature of approximately $50^{\circ} \mathrm{C}$. It has been determined that the welding circuits around the tunnel have sufficient load capability to allow sector-by-sector bakeouts in the tunnel.

\subsubsection{Anticipated Ion Pump Performance}

The standard lumped ion pumps being considered for use in the Recycler ring are $30 \mathrm{l} / \mathrm{s}$ diode ion sputter pumps. The speed of these pumps is a function of the pressure. At a pressure of $10^{-8}$ Torr the pumping speed is $100 \%$ of the advertised capacity. At a pressure of $10^{-9}$ Torr the pumping speed falls to $75 \%$, and at $10^{-10}$ Torr the pumping speed is reduced to $55 \%$ of the rated capacity.

Different gasses are pumped at different speeds. Table 4.5.3 lists these pumping speeds for the same $30 \mathrm{l} / \mathrm{s}$ diode ion sputter pumps. The dominant vacuum gas hydrogen expected at pressures below $10^{-9}$ Torr is pumped at a rate more than twice the nominal speed. Table 4.5.4 is a repeat of the calculations in table 4.5.1 assuming only hydrogen contaminates the vacuum. Both the pumping speed and the conductance were adjusted to reflect the vacuum performance. 
Table 4.5.3: Pumping speed for a number of common gasses in high vacuum systems. The pump is rated as a $30 \mathrm{l} / \mathrm{s}$ diode pump.

\begin{tabular}{|l|c|}
\hline Type of Gas & Speed $(1 / \mathrm{s})$ \\
\hline \hline Nitrogen & 35 \\
\hline Helium & 2.5 \\
\hline Carbon Monoxide & 35 \\
\hline Hydrogen & $74-100$ \\
\hline
\end{tabular}

Table 4.5.4: Results using vacuum calculations assuming hydrogen is the only residual gas and a cleaned and baked beam pipe whose width is equal to the Main Injector good field width criterion.

\begin{tabular}{|c|c|c|c|c|}
\hline Parameter & $\begin{array}{l}\text { 1 Pump/ } \\
\text { Half Cell }\end{array}$ & $\begin{array}{l}2 \text { Pumps/ } \\
\text { Half Cell }\end{array}$ & $\begin{array}{l}\text { 3 Pumps/ } \\
\text { Half Cell }\end{array}$ & $\begin{array}{l}2 \text { Pumps } \\
\text { @Bellow }\end{array}$ \\
\hline Lumped pump pumping speed $(1 / \mathrm{s})$ & 70 & 65 & 60 & $2 \times 65$ \\
\hline Specific outgassing rate $\left(\mathrm{T}-\mathrm{l} / \mathrm{s}-\mathrm{cm}^{2}\right)$ & $1 \times 10^{-12}$ & $1 \times 10^{-12}$ & $1 \times 10^{-12}$ & $1 \times 10^{-12}$ \\
\hline Distance between pumps (m) & 17.3 & 8.65 & 5.77 & 17.3 \\
\hline Total width of elliptical pipe (in.) & 3.75 & 3.75 & 3.75 & 3.75 \\
\hline Total height of elliptical pipe (in.) & 1.75 & 1.75 & 1.75 & 1.75 \\
\hline Specific conductance $(\mathrm{m}-1 / \mathrm{s})$ & 146.6 & 146.6 & 146.6 & 146.6 \\
\hline Pressure at lumped pumps (T) & $5.4 \times 10^{-10}$ & $2.9 \times 10^{-10}$ & $2.1 \times 10^{-10}$ & $2.9 \times 10^{-10}$ \\
\hline Pressure midway between pumps & $11 \times 10^{-10}$ & $4.3 \times 10^{-10}$ & $2.7 \times 10^{-10}$ & $8.5 \times 10^{-10}$ \\
\hline Average pressure $(\mathrm{T})$ & $9.2 \times 10^{-10}$ & $3.9 \times 10^{-10}$ & $2.5 \times 10^{-10}$ & $6.7 \times 10^{-10}$ \\
\hline
\end{tabular}

\subsection{Injection and Extraction}

There are four injection/extraction lines going in to and out of the Recycler ring. The first is the $8 \mathrm{GeV}$ antiproton injection line at MI-52. The second is the dedicated proton injection line at MI-10. The third is the antiproton extraction line from the Recycler to the Main Injector, probably at MI-30. Finally, the fourth line is the proton extraction line from the Recycler to the Main Injector, also at MI-30.

In addition, a proton abort line at MI-40 is needed for Recycler commissioning and other operations in which high flux proton injections are anticipated. The Main Injector abort block will also be used for the Recycler.

\subsection{RF System}

A broadband RF system is needed to create the longitudinal gap in the antiproton distribution required for stacking. In addition, the same RF system with reversed phase maintains the bunching of the coalesced protons while they are cooled longitudinally in the Recycler. Third, the broadband RF system is used to segment the antiprotons for extraction into the Tevatron Collider. Finally, a $53 \mathrm{MHz}$ voltage is applied to the beam to generate a current modulation which the beam position monitors can detect in order to measure the closed orbit of the Recycler.

Given that only about $200 \mathrm{~V}$ are required, a standard $50 \Omega$ terminated gap driven by a solid state driver is sufficient. With a minimum frequency of $100 \mathrm{kHz}$ and a maximum frequency of $10 \mathrm{MHz}$, standard amplifiers and cables are readily available. 


\subsection{Dampers and Feedback}

The dominant instabilities expected in the Recycler ring are the transverse resistive wall and longitudinal self-bunching or microwave instability. The growth rate of the resistive wall instability is proportional to the antiproton beam current. The longitudinal self-bunching or microwave instability will occur when the electron cooling reduces the momentum spread of the beam to the point where the beam will begin to spontaneously bunch at a frequency somewhere in the $\mathrm{MHz}$ or $\mathrm{GHz}$ range.

The broadband RF cavity connected to a resistive wall monitor should form the basis for any longitudinal feedback which is required. Given assumptions of longitudinal impedance from the kicker magnets, bellows, and beam position monitors, the gain and power of this system have yet to be analyzed

The resistive wall instability can be cured by a damper system very similar to the one which already exists in the Accumulator.

\subsection{Beam Instrumentation}

To first order the beam instrumentation in the Recycler ring is identical to that in the Main Injector. Reliability is crucial, so flying wires are probably not suitable. Instead, residual gas ionization monitors should be used to monitor the transverse beam emittance The beam position monitors will be at the mid-point of each half cell, where there is plenty of extra room in this lattice with gradient magnets.

\subsection{Correction Systems}

The criterion used to determine the correction magnet system are high reliability, low cost, and simple installation. The multipoles which will be corrected are dipole, quadrupole, and sextupole.

The dipole correction system is only used for short term harmonic correction to get around tight spots due to beam pipe misalignment, injection and extraction, or electron cooling. For the most part the closed orbit of the Recycler ring will be determined by magnet placement, similar to the Booster and Main Injector. The technology to be used is also permanent magnets. The field variability is accomplished by rotating two separate dipoles which are next to each other. This method of "paraphasing" can generate fields anywhere from twice the field of a single magnet (either positive or negative) to zero. The rotation is accomplished by using stepping motors on rotational stages which are supporting the individual permanent magnet dipole magnets. With such an arrangement the closed orbit of the Recycler remains unchanged during power outages and other failures.

The specific locations which need correction dipoles are at the antiproton injection point (MI-52), the Main Injector/Recycler proton/antiproton transfer area (MI-22), and the electron cooling straight section (MI-30). Each of these regions requires 4 pairs of correction dipoles to align the position and angle of the beams during transfer or to align the antiproton beam with the electron cooler.

The quadrupole correction system is used to adjust the horizontal and vertical tunes. Using the same paraphasing technique, these quads can also be used to adjust the global coupling of the accelerator. 
Finally, there may be some need to adjust the chromaticity in order to minimize the tune spread of the beam if that problem every arises.

\subsection{Intrabeam Scattering}

Electron cooling reduces both the longitudinal and transverse emittances of the antiproton beam. As the phase-space density of the beam increases, internal Coulomb scattering begins to heat the beam, until an equilibrium beam size and momentum spread is established. The equilibrium sizes are current dependent, and determine the exact means by which coalesced beams are extracted and accelerated through transition in the Main Injector.

\subsection{Beam Instabilities}

The dominant instabilities expected in the Recycler ring are the transverse resistive wall and longitudinal self-bunching or microwave. The growth rate of the resistive wall instability is proportional to the antiproton beam current. The longitudinal self-bunching or microwave instability will occur when the electron cooling reduces the momentum spread of the beam until the point where the beam will begin to spontaneously bunch at some frequency somewhere in the $\mathrm{MHz}$ or $\mathrm{GHz}$ range. 